\title{
NOTES ON ABSOLUTE HODGE CLASSES
}

\author{
FRANÇOIS CHARLES AND CHRISTIAN SCHNELL
}

\section{INTRODUCTION}

Absolute Hodge classes first appear in Deligne's proof of the Weil conjectures for K3 surfaces in [14] and are explicitly introduced in [16. The notion of absolute Hodge classes in the singular cohomology of a smooth projective variety stands between that of Hodge classes and classes of algebraic cycles. While it is not known whether absolute Hodge classes are algebraic, their definition is both of an analytic and arithmetic nature.

The paper [14] contains one of the first appearances of the notion of motives, and is among the first unconditional applications of motivic ideas. Part of the importance of the notion of absolute Hodge classes is indeed to provide an unconditional setting for the application of motivic ideas. The papers [14, 17] and [1, among others, give examples of this train of thoughts. The book [22] develops a theory of mixed motives based on absolute Hodge classes.

In these notes, we survey the theory of Hodge classes. The first section of these notes deals with algebraic de Rham cohomology and cycle classes. As proved by Grothendieck, the singular cohomology groups of a complex algebraic variety can be computed using suitable algebraic de Rham complexes. This provides an algebraic device for computing topological invariants of complex algebraic varieties.

The preceding construction is the main tool behind the definition of Hodge classes, which is the object of section 2. Indeed, this allows to consider Galois actions on the singular cohomology groups with complex coefficients of a complex algebraic variety. In section 2, we discuss the definition of absolute Hodge classes. We try to investigate two aspects of this subject. The first one pertains to the Hodge conjecture. Absolute Hodge classes make it possible to shed some light on the problem of the algebraicity of Hodge classes, and allow us to isolate the number-theoretic content of the Hodge conjecture. The second aspect we hint at is the motivic meaning of absolute Hodge classes. While we do not discuss the construction of motives for absolute Hodge classes as in [17, we show various functoriality and semi-simplicity properties of absolute Hodge classes which lie behind the more general motivic constructions cited above. We try to phrase our results so as to get results and proofs which are valid for André's theory of motivated cycles as in [1]. While we do not define motivated cycles, some of our proofs are very much inspired by this paper.

The third section deals with variational properties of absolute Hodge classes. After stating the variational Hodge conjecture, we prove Deligne's principle B as in [16 which one of the main technical tools of the paper. In the remainder of the section, we discuss consequences of the algebraicity of Hodge bundles and of the Galois action on relative de Rham cohomology. Following 31, we investigate the meaning of the theorem of Deligne-Cattani-Kaplan on the algebraicity of Hodge loci, see [10, and discuss the link between Hodge classes being absolute and the field of definition of Hodge loci. 
The last two sections are devoted to important examples of absolute Hodge classes. Section 4 discusses the Kuga-Satake correspondence following Deligne in 14. In section 5, we give a full proof of Deligne's theorem which states that Hodge classes on abelian varieties are absolute, see [16.

In writing these notes, we did not seek efficiency at all costs. Indeed, we did not necessarily prove properties of absolute Hodge cycles in the shortest way possible, but we rather chose to emphasize a variety of techniques and ideas.

Acknowledgements. This text is an expanded version of five lectures given at the ICTP summer school on Hodge theory in Trieste in June 2010. The first two lectures were devoted to absolute Hodge cycles and arithmetic aspects of the Hodge conjecture. The remaining three lectures outlined Deligne's proof that every Hodge class on an abelian variety is an absolute Hodge class. We would like to thank the organizers for this very nice and fruitful summer school.

Claire Voisin was supposed to give these lectures in Trieste, but she could not attend. It would be hard to acknowledge enough the influence of her work on these notes and the lectures we gave. We are grateful for her allowing us to use the beautiful survey 33] for our lectures and thank her sincerely. We also want to thank Matt Kerr for giving one of the lectures.

\section{Contents}

Introduction

Acknowledgements

1. Algebraic de Rham cohomology

1.1. Algebraic de Rham cohomology

1.2. Cycle classes

2. Absolute Hodge classes

2.1. Algebraic cycles and the Hodge conjecture

2.2. Galois action, algebraic de Rham cohomology and absolute Hodge classes

2.3. Variations on the definition and some functoriality properties

2.4. Classes coming from the standard conjectures and polarizations

2.5. Absolute Hodge classes and the Hodge conjecture

3. Absolute Hodge classes in families

3.1. The variational Hodge conjecture and the global invariant cycle theorem

3.2. Deligne's Principle B

3.3. The locus of Hodge classes

3.4. Galois action on relative de Rham cohomology

3.5. The field of definition of the locus of Hodge classes

4. The Kuga-Satake construction

4.1. Recollection on Spin groups

4.2. Spin representations

4.3. Hodge structures and the Deligne torus

4.4. From weight two to weight one

4.5. The Kuga-Satake correspondence is absolute

5. Deligne's theorem on Hodge classes on abelian varieties $\quad 33$

5.1. Overview

5.2. Hodge structures of CM-type 
5.3. Reduction to abelian varieties of CM-type 38

5.4. Background on hermitian forms 40

5.5. Construction of split Weil classes 42

5.6. André's theorem and reduction to split Weil classes 43

5.7. Split Weil classes are absolute 45

References 49

\section{Algebraic de Rham cohomology}

Shortly after Hironaka's paper on resolutions of singularities had appeared, Grothendieck observed that the cohomology groups of a complex algebraic variety can be computed algebraically. More precisely, he showed that on a nonsingular $n$-dimensional algebraic variety $X$ (of finite type over the field of complex numbers $\mathbb{C}$ ), the hypercohomology of the algebraic de Rham complex

$$
\mathscr{O}_{X} \rightarrow \Omega_{X / \mathbb{C}}^{1} \rightarrow \cdots \rightarrow \Omega_{X / \mathbb{C}}^{n}
$$

is isomorphic to the singular cohomology $H^{*}\left(X^{\text {an }}, \mathbb{C}\right)$ of the complex manifold corresponding to $X$. Grothendieck's theorem makes it possible to ask arithmetic questions in Hodge theory, and is the founding stone for the theory of absolute Hodge classes. In this lecture, we briefly review Grothendieck's theorem, as well as the construction of cycle classes in algebraic de Rham cohomology.

1.1. Algebraic de Rham cohomology. We begin by describing algebraic de Rham cohomology in a more general setting. Let $X$ be a nonsingular quasi-projective variety, defined over a field $K$ of characteristic zero. This means that we have a morphism $X \rightarrow \operatorname{Spec} K$, and we let $\Omega_{X / K}^{1}$ denote the sheaf of Kähler differentials on $X$. We also define $\Omega_{X / K}^{i}=\bigwedge^{i} \Omega_{X / K}^{1}$.

Definition 1. The algebraic de Rham cohomology of $X \rightarrow K$ consists of the $K$-vector spaces

$$
H^{i}(X / K)=\mathbb{H}^{i}\left(\mathscr{O}_{X} \rightarrow \Omega_{X / K}^{1} \rightarrow \cdots \rightarrow \Omega_{X / K}^{n}\right),
$$

where $n=\operatorname{dim} X$.

This definition is compatible with field extensions, for the following reason. Given a field extension $K \subseteq L$, we let $X_{L}=X \times_{\operatorname{Spec} K}$ Spec $L$ denote the variety obtained from $X$ by extension of scalars. Since $\Omega_{X_{L} / L}^{1} \simeq \Omega_{X / K}^{1} \otimes_{K} L$, we obtain $H^{i}\left(X_{L} / L\right) \simeq H^{i}(X / K) \otimes_{K} L$.

The algebraic de Rham complex $\Omega_{X / K}^{\bullet}$ is naturally filtered by the subcomplexes $\Omega_{X / K}^{\bullet \geq p}$, and we shall denote the induced filtration on its hypercohomology by

$$
F^{p} H^{i}(X / K)=\operatorname{Im}\left(\mathbb{H}^{i}\left(\Omega_{X / K}^{\bullet \geq p} \rightarrow \Omega_{X / K}^{\bullet}\right)\right.
$$

and refer to it as the Hodge filtration. We can now state Grothendieck's comparison theorem.

Theorem 2 (Grothendieck). Let $X$ be a nonsingular projective variety over $\mathbb{C}$, and let $X^{\text {an }}$ denote the associated complex manifold. Then there is a canonical isomorphism

$$
H^{i}(X / \mathbb{C}) \simeq H^{i}\left(X^{\text {an }}, \mathbb{C}\right),
$$

and under this isomorphism, $F^{p} H^{i}(X / \mathbb{C}) \simeq F^{p} H^{i}\left(X^{\text {an }}, \mathbb{C}\right)$ gives the Hodge filtration on singular cohomology. 
Proof. The theorem is a consequence of the GAGA theorem of Serre. Let $\mathscr{O}_{X^{\text {an }}}$ denote the sheaf of holomorphic functions on the complex manifold $X^{\text {an }}$. We then have a morphism $\pi:\left(X^{\text {an }}, \mathscr{O}_{X^{\text {an }}}\right) \rightarrow$ $\left(X, \mathscr{O}_{X}\right)$ of locally ringed spaces. For any coherent sheaf $\mathscr{F}$ on $X$, the associated coherent analytic sheaf on $X^{\text {an }}$ is given by $\mathscr{F}^{\text {an }}=\pi^{*} \mathscr{F}$, and according to Serre's theorem, $H^{i}(X, \mathscr{F}) \simeq H^{i}\left(X^{\text {an }}, \mathscr{F}^{\text {an }}\right)$.

It is easy to see from the local description of the sheaf of Kähler differentials that $\left(\Omega_{X / \mathbb{C}}^{1}\right)^{\text {an }}=$ $\Omega_{X^{\text {an }}}^{1}$. This implies that $H^{q}\left(X, \Omega_{X / \mathbb{C}}^{p}\right) \simeq H^{q}\left(X^{\text {an }}, \Omega_{X^{\text {an }}}^{p}\right)$ for all $p, q \geq 0$. Now pullback via $\pi$ induces homomorphisms $\mathbb{H}^{i}\left(\Omega_{X / \mathbb{C}}^{\bullet}\right) \rightarrow \mathbb{H}^{i}\left(\Omega_{X^{\text {an }}}^{\bullet}\right)$, which are isomorphism by Serre's theorem. Indeed, the groups on the left are computed by a spectral sequence with $E_{2}^{p, q}(X)=H^{q}\left(X, \Omega_{X / \mathbb{C}}^{p}\right)$, and the groups on the right by a spectral sequence with terms $E_{2}^{p, q}\left(X^{\text {an }}\right)=H^{q}\left(X^{\text {an }}, \Omega_{X^{\text {an }}}^{p}\right)$, and the two spectral sequences are isomorphic starting from the $E_{2}$-page. By the Poincaré lemma, the holomorphic de Rham complex $\Omega_{X \text { an }}^{\bullet}$ is a resolution of the constant sheaf $\mathbb{C}$, and therefore $H^{i}\left(X^{\text {an }}, \mathbb{C}\right) \simeq \mathbb{H}^{i}\left(\Omega_{X^{\text {an }}}^{\bullet}\right)$. Putting everything together, we obtain a canonical isomorphism

$$
H^{i}(X / \mathbb{C}) \simeq H^{i}\left(X^{\text {an }}, \mathbb{C}\right)
$$

Since the Hodge filtration on $H^{i}\left(X^{\text {an }}, \mathbb{C}\right)$ is induced by the naive filtration on the complex $\Omega_{X^{\bullet}}^{\text {an }}$, the second assertion follows by the same argument.

Remark 3. A similar result holds when $X$ is nonsingular and quasi-projective. Using resolution of singularities, one can find a nonsingular variety $\bar{X}$ and a divisor with normal crossing singularities, such that $X=\bar{X}-D$. Using differential forms with at worst logarithmic poles along $D$, one still has

$$
H^{i}\left(X^{\mathrm{an}}, \mathbb{C}\right) \simeq \mathbb{H}^{i}\left(\Omega \overline{\bar{X}}^{a n}\left(\log D^{\mathrm{an}}\right)\right) \simeq \mathbb{H}^{i}\left(\Omega \Omega_{\bar{X} / \mathbb{C}}(\log D)\right) ;
$$

under this isomorphism, the Hodge filtration is again induced by the naive filtration on the logarithmic de Rham complex $\Omega_{\bar{X}^{a n}}^{\bullet}\left(\log D^{\text {an }}\right)$. Since algebraic differential forms on $X$ have at worst poles along $D$, it can further be shown that those groups are still isomorphic to $H^{i}(X / \mathbb{C})$.

The general case of a possibly singular quasi-projective variety is dealt with in [15]. It involves the previous construction together with simplicial techniques.

Now suppose that $X$ is defined over a subfield $K \subseteq \mathbb{C}$. Then the complex vector space $H^{i}\left(X^{\text {an }}, \mathbb{C}\right)$ has two additional structures: a $\mathbb{Q}$-structure, coming from the universal coefficients theorem

$$
H^{i}\left(X^{\text {an }}, \mathbb{C}\right) \simeq H^{i}\left(X^{\text {an }}, \mathbb{Q}\right) \otimes_{\mathbb{Q}} \mathbb{C},
$$

and a $K$-structure, coming from Grothendieck's theorem

$$
H^{i}\left(X^{\text {an }}, \mathbb{C}\right) \simeq H^{i}(X / K) \otimes_{K} \mathbb{C} .
$$

In general, these two structures are not compatible with each other. It should be noted that the Hodge filtration is defined over $K$.

The same construction works in families to show that Hodge bundles and the Gauss-Manin connection are algebraic. Let $f: X \rightarrow B$ be a smooth projective morphism of varieties over $\mathbb{C}$. For each $i$, it determines a variation of Hodge structure on $B$ whose underlying vector bundle is

$$
\mathcal{H}^{i}=R^{i} f_{*} \mathbb{Q} \otimes_{\mathbb{Q}} \mathscr{O}_{B^{\text {an }}} \simeq \mathbb{R}^{i} f_{*}^{\text {an }} \Omega_{X^{\text {an }} / B^{\text {an }}} \simeq\left(\mathbb{R}^{i} f_{*} \Omega_{X / B}^{\bullet}\right)^{\text {an }} .
$$

By the relative version of Grothendieck's theorem, the Hodge bundles are given by

$$
F^{p} \mathcal{H}^{i} \simeq\left(\mathbb{R}^{i} f_{*} \Omega_{X / B}^{\bullet \geq p}\right)^{\text {an }} .
$$


Katz and Oda have shown in [23] that the Gauss-Manin connection $\nabla: \mathcal{H}^{i} \rightarrow \Omega_{B^{\text {an }}}^{1} \otimes \mathcal{H}^{i}$ can also be constructed algebraically. Starting from the exact sequence

$$
0 \rightarrow f^{*} \Omega_{B / \mathbb{C}}^{1} \rightarrow \Omega_{X / \mathbb{C}}^{1} \rightarrow \Omega_{B / \mathbb{C}}^{1} \rightarrow 0
$$

let $L^{r} \Omega_{X / \mathbb{C}}^{i}=f^{*} \Omega_{B / \mathbb{C}}^{r} \wedge \Omega_{X / \mathbb{C}}^{i-r}$. We get a short exact sequence of complexes

$$
0 \rightarrow f^{*} \Omega_{B / \mathbb{C}}^{1} \otimes \Omega_{X / B}^{\bullet-1} \rightarrow \Omega_{X / \mathbb{C}}^{\bullet} / L^{2} \Omega_{X / \mathbb{C}}^{\bullet} \rightarrow \Omega_{X / B}^{\bullet} \rightarrow 0,
$$

and hence a connecting morphism

$$
\mathbb{R}^{i} f_{*} \Omega_{X / B}^{\bullet} \rightarrow \mathbb{R}^{i+1} f_{*}\left(f^{*} \Omega_{B / \mathbb{C}}^{1} \otimes \Omega_{X / B}^{\bullet-1}\right) \simeq \Omega_{B / \mathbb{C}}^{1} \otimes \mathbb{R}^{i} f_{*} \Omega_{X / B}^{\bullet} .
$$

The theorem of Katz-Oda is that the associated morphism between analytic vector bundles is precisely the Gauss-Manin connection $\nabla$.

For our purposes, the most interesting conclusion is the following: if $f, X$, and $B$ are all defined over a subfield $K \subseteq \mathbb{C}$, then the same is true for the Hodge bundles $F^{p} \mathcal{H}^{i}$ and the Gauss-Manin connection $\nabla$. We shall make use of this fact later when discussing absolute Hodge classes and Deligne's Principle B.

1.2. Cycle classes. Let $X$ be a nonsingular projective variety over $\mathbb{C}$, of dimension $n=\operatorname{dim} X$. Let $Z \subseteq X$ be an algebraic subvariety of codimension $p$. It determines a cycle class

$$
\left[Z^{\mathrm{an}}\right] \in H^{2 p}\left(X^{\mathrm{an}}, \mathbb{Q}(p)\right)
$$

in Betti cohomology, as follows: Let $\widetilde{Z}$ be a resolution of singularities of $Z$, and let $\mu: \widetilde{Z} \rightarrow X$ denote the induced morphism. By Poincaré duality, the linear functional

$$
H^{2 n-2 p}\left(X^{\mathrm{an}}, \mathbb{Q}\right) \rightarrow \mathbb{Q}, \quad \alpha \mapsto \frac{1}{(2 \pi i)^{n-p}} \int_{\widetilde{Z}^{\text {an }}} \mu^{*}(\alpha)
$$

is represented by a unique class $\zeta \in H^{2 p}\left(X^{\text {an }}, \mathbb{Q}\right)$, with the property that

$$
\frac{1}{(2 \pi i)^{n-p}} \int_{\widetilde{Z}^{\text {an }}} \mu^{*}(\alpha)=\frac{1}{(2 \pi i)^{n}} \int_{X^{\text {an }}} \zeta \cup \alpha
$$

This class is clearly of type $(p, p)$, and therefore a Hodge class. To eliminate the annoying factor of $(2 \pi i)$, we define the cycle class of $Z$ to be $\left[Z^{\text {an }}\right]=(2 \pi i)^{p} \zeta$, which is now a rational Hodge class in the weight zero Hodge structure $H^{2 p}\left(X^{\text {an }}, \mathbb{Q}(p)\right)$.

An important fact is that one can also define a cycle class

$$
[Z] \in F^{p} H^{2 p}(X / \mathbb{C})
$$

in algebraic de Rham cohomology, and that the following comparison theorem holds.

Theorem 4. Under the isomorphism $H^{2 p}(X / \mathbb{C}) \simeq H^{2 p}\left(X^{\text {an }}, \mathbb{C}\right)$, we have

$$
[Z]=\left[Z^{\mathrm{an}}\right] .
$$

Consequently, if $Z$ and $X$ are both defined over a subfield $K \subseteq \mathbb{C}$, then the cycle class [ $\left.Z^{\text {an }}\right]$ is actually defined over the algebraic closure $\bar{K}$.

In the remainder of this lecture, our goal is to understand the construction of the algebraic cycle class, and where the factor $(2 \pi i)^{p}$ in the definition of the cycle class comes from. We shall first look at a nice special case, due to Bloch. Assume for now that $Z$ is a local complete intersection of codimension $p$. This means that $X$ can be covered by open sets $U$, with the property that 
$Z \cap U=V\left(f_{1}, \ldots, f_{p}\right)$ is the zero scheme of $p$ regular functions $f_{1}, \ldots, f_{p}$. Then $U-Z \cap U$ is covered by the open sets $D\left(f_{1}\right), \ldots, D\left(f_{p}\right)$, and

$$
\frac{d f_{1}}{f_{1}} \wedge \cdots \wedge \frac{d f_{p}}{f_{p}}
$$

is a closed $p$-form on $D\left(f_{1}\right) \cap \cdots \cap D\left(f_{p}\right)$. Using Čech cohomology, it determines a class in

$$
H^{p-1}\left(U-Z \cap U, \Omega_{X / \mathbb{C}}^{p, \mathrm{cl}}\right)
$$

where $\Omega_{X / \mathbb{C}}^{p, \text { cl }}$ is the subsheaf of $\Omega_{X / \mathbb{C}}^{p}$ consisting of closed $p$-forms. Since we have a map of complexes $\Omega_{X / \mathbb{C}}^{p, \mathrm{cl}}[-p] \rightarrow \Omega_{X / \mathbb{C}}^{\bullet \geq p}$, we get

$$
H^{p-1}\left(U-Z \cap U, \Omega_{X / \mathbb{C}}^{p, \mathrm{cl}}\right) \rightarrow \mathbb{H}^{2 p-1}\left(U-Z \cap U, \Omega_{X / \mathbb{C}}^{\bullet \geq p}\right) \rightarrow \mathbb{H}_{Z \cap U}^{2 p}\left(\Omega_{X / \mathbb{C}}^{\bullet \geq p}\right) .
$$

One can show that the image of (11) in the cohomology group with supports on the right does not depend on the choice of local equations $f_{1}, \ldots, f_{p}$. (A good exercise is to prove this for $p=1$ and $p=2$.) It therefore defines a global section of the sheaf $\mathcal{H}_{Z}^{2 p}\left(\Omega_{X / \mathbb{C}}^{\bullet \geq p}\right)$. Using that $\mathcal{H}_{Z}^{i}\left(\Omega_{X / \mathbb{C}}^{\bullet \geq p}\right)=0$ for $i \leq 2 p-1$, we get from the local-to-global spectral sequence that

$$
\mathbb{H}_{Z}^{2 p}\left(\Omega_{X / \mathbb{C}}^{\bullet \geq p}\right) \simeq H^{0}\left(X, \mathcal{H}_{Z}^{2 p}\left(\Omega_{X / \mathbb{C}}^{\bullet \geq p}\right)\right)
$$

In this way, we obtain a well-defined class in $\mathbb{H}_{Z}^{2 p}\left(\Omega_{X / \mathbb{C}}^{\bullet \geq p}\right)$, and hence in the algebraic de Rham cohomology $\mathbb{H}^{2 p}\left(\Omega_{X / \mathbb{C}}^{\bullet \geq p}\right)=F^{p} H^{2 p}(X / \mathbb{C})$.

For the general case, one uses the theory of Chern classes, which associates to a locally free sheaf $\mathscr{E}$ of rank $r$ a sequence of Chern classes $c_{1}(\mathscr{E}), \ldots, c_{r}(\mathscr{E})$. We recall their construction in Betti cohomology and in algebraic de Rham cohomology.

First, consider the case of an algebraic line bundle $\mathscr{L}$; as usual, we denote the associated holomorphic line bundle by $\mathscr{L}^{\text {an }}$. The first Chern class $c_{1}\left(\mathscr{L}^{\text {an }}\right) \in H^{2}\left(X^{\text {an }}, \mathbb{Z}(1)\right)$ can be defined using the exponential sequence

$$
0 \rightarrow \mathbb{Z}(1) \rightarrow \mathscr{O}_{X^{\text {an }}} \stackrel{\exp }{\longrightarrow} \mathscr{O}_{X^{\text {an }}}^{*} \rightarrow 0
$$

The isomorphism class of $\mathscr{L}^{\text {an }}$ belongs to $H^{1}\left(X^{\text {an }}, \mathscr{O}_{X^{\text {an }}}^{*}\right)$, and $c_{1}\left(\mathscr{L}^{\text {an }}\right)$ is the image of this class under the connecting homomorphism.

To relate this to differential forms, cover $X$ by open subsets $U_{i}$ on which $\mathscr{L}^{\text {an }}$ is trivial, and let $g_{i j} \in \mathscr{O}_{X}^{*}{ }^{\text {an }}\left(U_{i} \cap U_{j}\right)$ denote the holomorphic transition functions for this cover. If each $U_{i}$ is simply connected, say, then we can write $g_{i j}=e^{f_{i j}}$, and then

$$
f_{j k}-f_{i k}+f_{i j} \in \mathbb{Z}(1)
$$

form a 2-cocycle that represents $c_{1}\left(\mathscr{L}^{\text {an }}\right)$. Its image in $H^{2}\left(X^{\text {an }}, \mathbb{C}\right) \simeq \mathbb{H}^{2}\left(\Omega_{X^{\text {an }}}^{\bullet}\right)$ is cohomologous to the class of the 1 -cocycle $d f_{i j}$ in $H^{1}\left(X^{\text {an }}, \Omega_{X^{\text {an }}}^{1}\right)$. But $d f_{i j}=d g_{i j} / g_{i j}$, and so $c_{1}\left(\mathscr{L}^{\text {an }}\right)$ is also represented by the cocycle $d g_{i j} / g_{i j}$. This explains the special case $p=1$ in Bloch's construction.

To define the first Chern class of $\mathscr{L}$ in algebraic de Rham cohomology, we use the fact that a line bundle is also locally trivial in the Zariski topology. If $U_{i}$ are Zariski-open sets on which $\mathscr{L}$ is trivial, and $g_{i j} \in \mathscr{O}_{X}^{*}\left(U_{i} \cap U_{j}\right)$ denotes the corresponding transition functions, we can define $c_{1}(\mathscr{L}) \in$ $F^{1} H^{2}(X / \mathbb{C})$ as the hypercohomology class determined by the cocycle $d g_{i j} / g_{i j}$. In conclusion, we then have $c_{1}(\mathscr{L})=c_{1}\left(\mathscr{L}^{\text {an }}\right)$ under the isomorphism in Grothendieck's theorem.

Now suppose that $\mathscr{E}$ is a locally free sheaf of rank $r$ on $X$. On the associated projective bundle $\pi: \mathbb{P}(\mathscr{E}) \rightarrow X$, we have a universal line bundle $\mathscr{O}_{\mathscr{E}}(1)$, together with a surjection from $\pi^{*} \mathscr{E}$. In 
Betti cohomology, we have

$$
H^{2 r}\left(\mathbb{P}\left(\mathscr{E}{ }^{\text {an }}\right), \mathbb{Z}(r)\right)=\bigoplus_{i=0}^{r-1} \xi^{i} \cdot \pi^{*} H^{2 r-2 i}\left(X^{\text {an }}, \mathbb{Z}(r-i)\right),
$$

where $\xi \in H^{2}\left(\mathbb{P}\left(\mathscr{E}^{\text {an }}\right), \mathbb{Z}(1)\right)$ denotes the first Chern class of $\mathscr{O}_{\mathscr{E}}(1)$. Consequently, there are unique classes $c_{k} \in H^{2 k}\left(X^{\text {an }}, \mathbb{Z}(k)\right)$ that satisfy the relation

$$
\xi^{r}-\pi^{*}\left(c_{1}\right) \cdot \xi^{r-1}+\pi^{*}\left(c_{2}\right) \cdot \xi^{r-2}+\cdots+(-1)^{r} \pi^{*}\left(c_{r}\right)=0,
$$

and the $k$-th Chern class of $\mathscr{E}^{\text {an }}$ is defined to be $c_{k}\left(\mathscr{E}^{\text {an }}\right)=c_{k}$. The same construction can be carried out in algebraic de Rham cohomology, producing Chern classes $c_{k}(\mathscr{E}) \in F^{k} H^{2 k}(X / \mathbb{C})$. It follows easily from the case of line bundles that we have

$$
c_{k}(\mathscr{E})=c_{k}\left(\mathscr{E}^{\mathrm{an}}\right)
$$

under the isomorphism in Grothendieck's theorem.

Since coherent sheaves admit finite resolutions by locally free sheaves, it is possible to define Chern classes for arbitrary coherent sheaves. One consequence of the Riemann-Roch theorem is the equality

$$
\left[Z^{\text {an }}\right]=\frac{(-1)^{p-1}}{(p-1) !} c_{p}\left(\mathscr{O}_{Z^{\text {an }}}\right) \in H^{2 p}\left(X^{\text {an }}, \mathbb{Q}(p)\right) .
$$

Thus it makes sense to define the cycle class of $Z$ in algebraic de Rham cohomology by the formula

$$
[Z]=\frac{(-1)^{p-1}}{(p-1) !} c_{p}\left(\mathscr{O}_{Z}\right) \in F^{p} H^{2 p}(X / \mathbb{C}) .
$$

It follows that $[Z]=\left[Z^{\text {an }}\right]$, and so the cycle class of $Z^{\text {an }}$ can indeed be constructed algebraically, as claimed.

Exercise 5. Let $X$ be a nonsingular projective variety defined over $\mathbb{C}$, let $D \subseteq X$ be a nonsingular hypersurface, and set $U=X-D$. One can show that $H^{i}(U / \mathbb{C})$ is isomorphic to the hypercohomology of the $\log$ complex $\Omega_{X / \mathbb{C}}^{\bullet}(\log D)$. Use this to construct a long exact sequence

$$
\cdots \rightarrow H^{i-2}(D) \rightarrow H^{i}(X) \rightarrow H^{i}(U) \rightarrow H^{i-1}(D) \rightarrow \cdots
$$

for the algebraic de Rham cohomology groups. Conclude by induction on the dimension of $X$ that the restriction map

$$
H^{i}(X / \mathbb{C}) \rightarrow H^{i}(U / \mathbb{C})
$$

is injective for $i \leq 2 \operatorname{codim} Z-1$, and an isomorphism for $i \leq 2 \operatorname{codim} Z-2$.

\section{Absolute Hodge Classes}

In this section, we introduce the notion of absolute Hodge classes in the cohomology of a complex algebraic variety. While Hodge theory applies to general compact Kähler manifolds, absolute Hodge classes are brought in as a way to deal with cohomological properties of a variety coming from its algebraic structure.

This circle of ideas is closely connected to the motivic philosophy as envisioned by Grothendieck. One of the goals of this text is to hopefully give a hint of how absolute Hodge classes can allow one to give unconditional proofs for results of a motivic flavor. 
2.1. Algebraic cycles and the Hodge conjecture. As an example of the need for a suitable structure on the cohomology of a complex algebraic variety that uses more than usual Hodge theory, let us first discuss some aspects of the Hodge conjecture.

Let $X$ be a smooth projective variety over $\mathbb{C}$. The singular cohomology groups of $X$ are endowed with pure Hodge structures such that for any integer $p, H^{2 p}(X, \mathbb{Z}(p))$ has weight 0 . We denote by $H d g^{p}(X)$ the group of Hodge classes in $H^{2 p}(X, \mathbb{Z}(p))$.

As we showed earlier, if $Z$ is a subvariety of $X$ of codimension $p$, its cohomology class $[Z]$ in $H^{2 p}(X, \mathbb{Z}(p))$ is a Hodge class. The Hodge conjecture states that the cohomology classes of subvarieties of $X$ span the $\mathbb{Q}$-vector space generated by Hodge classes.

Conjecture 6. Let $X$ be a smooth projective variety over $\mathbb{C}$. For any nonnegative integer $p$, the subspace of degree $p$ rational Hodge classes

$$
H d g^{p}(X) \otimes \mathbb{Q} \subset H^{2 p}(X, \mathbb{Q}(p))
$$

is generated over $\mathbb{Q}$ by the cohomology classes of codimension $p$ subvarieties of $X$.

If $X$ is only assumed to be a compact Kähler manifold, the cohomology groups $H^{2 p}(X, \mathbb{Z}(p))$ still carry Hodge structures, and analytic subvarieties of $X$ still give rise to Hodge classes. While a general compact Kähler manifold can have very few analytic subvarieties, Chern classes of coherent sheaves also are Hodge classes on the cohomology of $X$.

Note that on a smooth projective complex variety, analytic subvarieties are algebraic by the GAGA principle of Serre, and that Chern classes of coherent sheaves are linear combinations of cohomology classes of algebraic subvarieties of $X$. Indeed, this is true for locally free sheaves and coherent sheaves on a smooth variety have finite free resolutions. This latter result is no longer true for general compact Kähler manifolds, and indeed Chern classes of coherent sheaves can generate a strictly larger subspace than that generated by the cohomology classes of analytic subvarieties.

These remarks show that the Hodge conjecture could be generalized to the Kähler setting by asking whether Chern classes of coherent sheaves on a compact Kähler manifold generate the space of Hodge classes. This would be the natural Hodge-theoretic framework for this question. However, the answer to this question is negative, as proved by Voisin in 29].

Theorem 7. There exists a compact Kähler manifold $X$ such that $H d g^{2}(X)$ is nontorsion while for any coherent sheaf $\mathcal{F}$ on $X, c_{2}(\mathcal{F})=0, c_{2}(\mathcal{F})$ denoting the second Chern class of $\mathcal{F}$.

The proof of the preceding theorem takes $X$ to be a general Weil torus. Weil tori are complex tori with a specific linear algebra condition which endows them with a nonzero space of Hodge classes. While here they provide a counterexample to the Hodge conjecture in the Kähler setting, they will be instrumental, in the projective case, to proving Deligne's theorem on absolute Hodge classes.

To our knowledge, there is no tentative formulation of a Hodge conjecture for compact Kähler manifolds. As a consequence, one has to bring ingredients from the algebraic world to tackle the Hodge conjecture for projective varieties.

2.2. Galois action, algebraic de Rham cohomology and absolute Hodge classes. The preceding paragraph suggests that the cohomology of projective complex varieties has a richer underlying structure than that of a general Kähler manifold. 
This brings us very close to the theory of motives, which Grothendieck created in the sixties as a way to encompass cohomological properties of algebraic varieties. Even though these notes won't use the language of motives, the motivic philosophy is pervasive to all the results we will state.

Historically, absolute Hodge classes were introduced by Deligne in order to make an unconditional use of motivic ideas. We will review his results in the next sections. The main starting point is, as we showed earlier, that the singular cohomology of a smooth proper complex algebraic variety with complex coefficients can be computed algebraically, using algebraic de Rham cohomology.

Indeed, let $X$ be a smooth proper complex algebraic variety defined over $\mathbb{C}$. We have a canonical isomorphism

$$
H^{*}\left(X^{a n}, \mathbb{C}\right) \simeq \mathbb{H}^{*}\left(\Omega_{X / \mathbb{C}}^{\bullet}\right)
$$

where $\Omega_{X / \mathbb{C}}^{\bullet}$ is the algebraic de Rham complex of the variety $X$ over $\mathbb{C}$. A striking consequence of this isomorphism is that the singular cohomology of the manifold $X^{a n}$ with complex definition can be computed algebraically. It follows that the topology of $\mathbb{C}$ is not needed in this computation, the field structure being sufficient. More generally, if $X$ is a smooth proper variety defined over any field $k$ of characteristic zero, the hypercohomology of the de Rham complex of $X$ over Spec $k$ gives a $k$-algebra which by definition is the algebraic de Rham cohomology of $X$ over $k$.

Now let $Z$ be an algebraic cycle of codimension $p$ in $X$. As we showed earlier, $Z$ has a cohomology class

$$
[Z] \in H^{2 p}\left(X^{a n}, \mathbb{Q}(p)\right)
$$

which is a Hodge class, that is, the image of $[Z]$ in $H^{2 p}\left(X^{a n}, \mathbb{C}(p)\right) \simeq H^{2 p}(X / \mathbb{C})(p)$ lies in

$$
F^{0} H^{2 p}(X / \mathbb{C})(p)=F^{p} H^{2 p}(X / \mathbb{C}) .
$$

Given any automorphism $\sigma$ of the field $\mathbb{C}$, we can form the conjugate variety $X^{\sigma}$ defined as the complex variety $X \times_{\sigma}$ Spec $\mathbb{C}$, that is, by the cartesian diagram

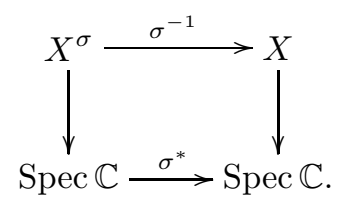

It is a smooth projective variety. If $X$ is defined by homogeneous polynomials $P_{1}, \ldots, P_{r}$ in some projective space, then $X^{\sigma}$ is defined by the conjugates of the $P_{i}$ by $\sigma$. In this case, the morphism from $X^{\sigma}$ to $X$ in the cartesian diagram sends the closed point with coordinates $\left(x_{0}: \ldots: x_{n}\right)$ to the closed point with homogeneous coordinates $\left(\sigma^{-1}\left(x_{0}\right): \ldots: \sigma^{-1}\left(x_{n}\right)\right)$, which allows us to denote it by $\sigma^{-1}$.

The morphism $\sigma^{-1}: X^{\sigma} \rightarrow X$ is an isomorphism of abstract schemes, but it is not a morphism of complex varieties. Pull-back of Kähler forms still induces an isomorphism between the de Rham complexes of $X$ and $X^{\sigma}$

$$
\left(\sigma^{-1}\right)^{*} \Omega_{X / \mathbb{C}}^{\bullet} \stackrel{\sim}{\rightarrow} \Omega_{X^{\sigma} / \mathbb{C}}^{\bullet}
$$

Taking hypercohomology, we get an isomorphism

$$
\left(\sigma^{-1}\right)^{*}: H^{*}(X / \mathbb{C}) \stackrel{\sim}{\rightarrow} H^{*}\left(X^{\sigma} / \mathbb{C}\right), \alpha \mapsto \alpha^{\sigma} .
$$


Note however that this isomorphism is not $\mathbb{C}$-linear, but $\sigma$-linear, that is, if $\lambda \in \mathbb{C}$, we have $(\lambda \alpha)^{\sigma}=\sigma(\lambda) \alpha^{\sigma}$. We thus get an isomorphism of complex vector spaces

$$
H^{*}(X / \mathbb{C}) \otimes_{\sigma} \mathbb{C} \stackrel{\sim}{\rightarrow} H^{*}\left(X^{\sigma} / \mathbb{C}\right)
$$

between the de Rham cohomology of $X$ and that of $X^{\sigma}$. Here the notation $\otimes_{\sigma}$ means that we are taking tensor product with $\mathbb{C}$ mapping to $\mathbb{C}$ via the morphism $\sigma$. Since this isomorphism comes from an isomorphism of the de Rham complexes, it preserves the Hodge filtration.

The preceding construction is compatible with the cycle map. Indeed, $Z$ being as before a codimension $p$ cycle in $X$, we can form its conjugate $Z^{\sigma}$ by $\sigma$. It is a codimension $p$ cycle in $X^{\sigma}$. The construction of the cycle class map in de Rham cohomology shows that we have

$$
\left[Z^{\sigma}\right]=[Z]^{\sigma}
$$

in $H^{2 p}\left(X^{\sigma} / \mathbb{C}\right)(p)$. It lies in $F^{0} H^{2 p}\left(X^{\sigma} / \mathbb{C}\right)(p)$.

Now as before $X^{\sigma}$ is a smooth projective complex variety, and its de Rham cohomology group $H^{2 p}\left(X^{\sigma} / \mathbb{C}\right)(p)$ is canonically isomorphic to the singular cohomology group $H^{2 p}\left(\left(X^{\sigma}\right)^{a n}, \mathbb{C}(p)\right)$. The cohomology class $\left[Z^{\sigma}\right]$ in $H^{2 p}\left(\left(X^{\sigma}\right)^{a n}, \mathbb{C}(p)\right) \simeq H^{2 p}\left(X^{\sigma} / \mathbb{C}\right)(p)$ is a Hodge class. This leads to the following definition.

Definition 8. Let $X$ be a smooth complex projective variety. Let $p$ be a nonnegative integer, and let $\alpha$ be an element of $H^{2 p}(X / \mathbb{C})(p)$. The cohomology class $\alpha$ is an absolute Hodge class if for every automorphism $\sigma$ of $\mathbb{C}$, the cohomology class $\alpha^{\sigma} \in H^{2 p}\left(\left(X^{\sigma}\right)^{\text {an }}, \mathbb{C}(p)\right) \simeq H^{2 p}\left(X^{\sigma} / \mathbb{C}(p)\right)$ is a Hodge clas $\$^{1}$.

The preceding discussion shows that the cohomology class of an algebraic cycle is an absolute Hodge class. Taking $\sigma=\operatorname{Id}_{\mathbb{C}}$, we see that absolute Hodge classes are Hodge classes.

Using the canonical isomorphism $H^{2 p}\left(X^{a n}, \mathbb{C}(p)\right) \simeq H^{2 p}(X / \mathbb{C})(p)$, we will say that a class in $H^{2 p}\left(X^{a n}, \mathbb{C}\right)$ is absolute Hodge if its image in $H^{2 p}(X / \mathbb{C})(p)$ is.

We can rephrase the definition of absolute Hodge cycles in a slightly more intrinsic way. Let $k$ be a field of characteristic zero, and let $X$ be a smooth projective variety defined over $k$. Assume that the cardinality of $k$ is less or equal to the cardinality of $\mathbb{C}$, so that there exist embeddings of $k$ into $\mathbb{C}$. Note that any variety defined over a field of characteristic zero is defined over such a field, as it is defined over a field generated over $\mathbb{Q}$ by a finite number of elements.

Definition 9. Let $p$ be a nonnegative integer, and let $\alpha$ be an element of the de Rham cohomology space $H^{2 p}(X / k)$. Let $\tau$ be an embedding of $k$ into $\mathbb{C}$, and let $\tau X$ be the complex variety obtained from $X$ by base change to $\mathbb{C}$. We say that $\alpha$ is a Hodge class relative to $\tau$ if the image of $\alpha$ in

$$
H^{2 p}(\tau X / \mathbb{C})=H^{2 p}(X / k) \otimes_{\tau} \mathbb{C}
$$

is a Hodge class. We say that $\alpha$ is absolute Hodge if it is a Hodge class relative to every embedding of $k$ into $\mathbb{C}$.

Let $\tau$ be any embedding of $k$ into $\mathbb{C}$. Since by standard field theory, any two embeddings of $k$ into $\mathbb{C}$ are conjugated by an automorphism of $\mathbb{C}$, it is straightforward to check that such a cohomology class $\alpha$ is absolute Hodge if and only if its image in $H^{2 p}(\tau X / \mathbb{C})$ is. Definition 9 has the advantage

\footnotetext{
${ }^{1}$ Since $H^{2 p}\left(\left(X^{\sigma}\right)^{a n}, \mathbb{C}\right)$ is only considered as a vector space here, the Tate twist might seem superfluous. We put it here to emphasize that the comparison isomorphism with de Rham cohomology contains a factor $(2 \pi i)^{-p}$.
} 
of not referring to automorphisms of $\mathbb{C}$, as it is impossible to exhibit one except for the identity and complex conjugation.

This definition allows us to work with absolute Hodge classes in a wider setting by using other cohomology theories. Even though we will be mainly concerned with de Rham cohomology in these notes, let us state what absolute Hodge classes are for étale cohomology.

Definition 10. Let $\bar{k}$ be an algebraic closure of $k$. Let $p$ be a nonnegative integer, $l$ a prime number, and let $\alpha$ be an element of the étale cohomology space $H^{2 p}\left(X_{\bar{k}}, \mathbb{Q}_{l}(p)\right)$. Let $\tau$ be an embedding of $\bar{k}$ into $\mathbb{C}$, and let $\tau X$ be the complex variety obtained from $X_{\bar{k}}$ by base change to $\mathbb{C}$. We say that $\alpha$ is a Hodge class relative to $\tau$ if the image of $\alpha$ in

$$
H^{2 p}\left((\tau X)^{a n}, \mathbb{Q}_{l}(p)\right) \simeq H^{2 p}\left(X_{\bar{k}}, \mathbb{Q}_{l}(p)\right)
$$

is a Hodge class, that is, if it lies in the rational subspace $H^{2 p}\left((\tau X)^{a n}, \mathbb{Q}(p)\right)$ of $H^{2 p}\left((\tau X)^{a n}, \mathbb{Q}_{l}(p)\right)$ and is a Hodge class. We say that $\alpha$ is absolute Hodge if it is a Hodge class relative to every embedding of $k$ into $\mathbb{C}$.

Remark 11. It is not clear whether an absolute Hodge class in the sense of definition 9 is always the first component of an absolute Hodge class in the sense of definition [10, see [16, Question 2.4.

Remark 12. It is possible to encompass crystalline cohomology in a similar framework, see for instance [4.

Remark 13. It is possible to work with absolute Hodge classes on more general varieties. Indeed, while the definitions we gave above only deal with the smooth projective case, the fact that the singular cohomology of any quasi-projective variety can be computed using suitable versions of algebraic de Rham cohomology - whether through logarithmic de Rham cohomology, algebraic de Rham cohomology on simplicial schemes or a combination of the two - makes it possible to consider absolute Hodge classes in the singular cohomology groups of a general complex variety.

Note here that if $H$ is a mixed Hodge structure defined over $\mathbb{Z}$ with weight filtration $W$ • and Hodge filtration $F^{\bullet}$, a Hodge class in $H$ is an element of $H_{\mathbb{Z}} \bigcap F^{0} H_{\mathbb{C}} \bigcap W_{0} H_{\mathbb{C}}$. One of the main specific features of absolute Hodge classes on quasi-projective varieties is that they can be found in the odd singular cohomology groups. Let us consider the one-dimensional case as an example. Let $C$ be a smooth complex projective curve, and let $D=\Sigma_{i} n_{i} P_{i}$ be a divisor of degree 0 on $C$. Let $Z$ be the support of $D$, and let $C^{\prime}$ be the complement of $Z$ in $C$. It is a smooth quasi-projective curve.

As in Exercise 5] we have an exact sequence

$$
0 \rightarrow H^{1}(C, \mathbb{Q}(1)) \rightarrow H^{1}\left(C^{\prime}, \mathbb{Q}(1)\right) \rightarrow H^{0}(Z, \mathbb{Q}) \rightarrow H^{2}(C, \mathbb{Q}(1)) .
$$

The divisor $D$ has a cohomology class $d \in H^{0}(Z, \mathbb{Q})$. Since the degree of $D$ is zero, $d$ maps to zero in $H^{2}(C, \mathbb{Q}(1))$. As a consequence, it comes from an element in $H^{1}\left(C^{\prime}, \mathbb{Q}(1)\right)$. Now it can be proved that there exists a Hodge class in $H^{1}\left(C^{\prime}, \mathbb{Q}(1)\right)$ mapping to $d$ if and only if some multiple of the divisor $D$ is rationally equivalent to zero.

In general, the existence of Hodge classes in extensions of mixed Hodge structures is to be related to Griffiths' Abel-Jacobi map, see [9. The problem of whether those are absolute Hodge classes is linked with problems pertaining to the Bloch-Beilinson filtration and comparison results with regulators in étale cohomology, see [22.

While we will not discuss here specific features of this problem, most of the results we will state in the pure case have extensions to the mixed case, see for instance [12. 
2.3. Variations on the definition and some functoriality properties. While the goal of these notes is neither to construct nor to discuss the category motives one can obtain using absolute Hodge classes, we will need to use functoriality properties of absolute Hodge classes that are very close to those motivic constructions. In this paragraph, we extend the definition of absolute Hodge classes to encompass morphisms, multilinear forms, etc. This almost amounts to defining motives for absolute Hodge classes as in [17. The next paragraph will be devoted to semi-simplicity results through the use of polarized Hodge structures.

The following generalizes Definition 9

Definition 14. Let $k$ be a field of characteristic zero with cardinality less or equal than the cardinality of $\mathbb{C}$. Let $\left(X_{i}\right)_{i \in I}$ and $\left(X_{j}\right)_{j \in J}$ be smooth projective varieties over $\mathbb{C}$, and let $\left(p_{i}\right)_{i \in I},\left(q_{j}\right)_{j \in J}$, $n$ be integers. Let $\alpha$ be an element of the tensor product

$$
\left(\bigotimes_{i \in I} H^{p_{i}}\left(X_{i} / k\right)\right) \otimes\left(\bigotimes_{j \in J} H^{q_{j}}\left(X_{j} / k\right)^{*}\right)(n)
$$

Let $\tau$ be an embedding of $k$ into $\mathbb{C}$. We say that $\alpha$ is a Hodge class relative to $\tau$ if the image of $\alpha$ in

$$
\left(\bigotimes_{i \in I} H^{p_{i}}\left(X_{i} / k\right)\right) \otimes\left(\bigotimes_{j \in J} H^{q_{j}}\left(X_{j} / k\right)^{*}\right)(n) \otimes_{\tau} \mathbb{C}=\left(\bigotimes_{i \in I} H^{p_{i}}\left(\tau X_{i} / \mathbb{C}\right)\right) \otimes\left(\bigotimes_{j \in J} H^{q_{j}}\left(\tau X_{j} / \mathbb{C}\right)^{*}\right)(n)
$$

is a Hodge class. We say that $\alpha$ is absolute Hodge if it is a Hodge class relative to every embedding of $k$ into $\mathbb{C}$.

As before, if $k=\mathbb{C}$, we can speak of absolute Hodge classes in the group

$$
\left(\bigotimes_{i \in I} H^{p_{i}}\left(X_{i}, \mathbb{Q}\right)\right) \otimes\left(\bigotimes_{j \in J} H^{q_{j}}\left(X_{j}, \mathbb{Q}\right)^{*}\right)(n)
$$

If $X$ and $Y$ are two smooth projective complex varieties, and if

$$
f: H^{p}(X, \mathbb{Q}(i)) \rightarrow H^{q}(Y, \mathbb{Q}(j))
$$

is a morphism of Hodge structures, we will say that $f$ is absolute Hodge, or is given by an absolute Hodge class, if the element corresponding to $f$ in

$$
H^{q}(Y, \mathbb{Q}) \otimes H^{p}(X, \mathbb{Q})^{*}(j-i)
$$

is an absolute Hodge class. Similarly, we can define what it means for a multilinear form, e.g., a polarization, to be absolute Hodge.

This definition allows us to exhibit elementary examples of Hodge classes as follows.

Example 15. Let $X$ be a smooth projective complex variety.

- Cup-product defines a map

$$
H^{p}(X, \mathbb{Q}) \otimes H^{q}(X, \mathbb{Q}) \rightarrow H^{p+q}(X, \mathbb{Q}) .
$$

This map is given by an absolute Hodge class.

- Poincaré duality defines an isomorphism

$$
H^{p}(X, \mathbb{Q}) \rightarrow H^{2 d-p}(X, \mathbb{Q}(d))^{*},
$$

where $d$ is the dimension of $X$. This map is given by an absolute Hodge class. 
Proof. This is formal. Let us write down the computations involved. Assume $X$ is defined over $k$ (which might be $\mathbb{C}$ ). We have a cup-product map

$$
H^{p}(X / k) \otimes H^{q}(X / k) \rightarrow H^{p+q}(X / k) .
$$

Let $\tau$ be an embedding of $k$ into $\mathbb{C}$. The induced map

$$
H^{p}(\tau X / \mathbb{C}) \otimes H^{q}(\tau X / \mathbb{C}) \rightarrow H^{p+q}(\tau X / \mathbb{C})
$$

is cup-product on the de Rham cohomology of $\tau X$. We know that cup-product on a smooth complex projective variety is compatible with Hodge structures, which shows that it is given by a Hodge class. The conclusion follows, and a very similar argument proves the result regarding Poincaré duality.

Morphisms given by absolute Hodge classes behave in a functorial way. The following properties are easy to prove, working as in the preceding example to track down compatibilities.

Proposition 16. Let $X, Y$ and $Z$ be smooth projective complex varieties, and let

$$
f: H^{p}(X, \mathbb{Q}(i)) \rightarrow H^{q}(Y, \mathbb{Q}(j)), g: H^{q}(Y, \mathbb{Q}(j)) \rightarrow H^{r}(Y, \mathbb{Q}(k))
$$

be morphisms of Hodge structures.

(1) If $f$ is induced by an algebraic correspondence, then $f$ is absolute Hodge.

(2) If $f$ and $g$ are absolute Hodge, then $g \circ f$ is absolute Hodge.

(3) Let

$$
f^{\dagger}: H^{2 d^{\prime}-q}\left(Y, \mathbb{Q}\left(d^{\prime}-j\right)\right) \rightarrow H^{2 d-p}(X, \mathbb{Q}(d-i))
$$

be the adjoint of $f$ with respect to Poincaré duality. Then $f$ is absolute Hodge if and only if $f^{\dagger}$ is absolute Hodge.

(4) If $f$ is an isomorphism, then $f$ is absolute Hodge if and only if $f^{-1}$ is absolute Hodge.

Note that the last property is not known to be true for algebraic correspondences. For those, it is equivalent to the Lefschetz standard conjecture, see the next paragraph. We will need a refinement of this property as follows.

Proposition 17. Let $X$ and $Y$ be smooth projective complex varieties, and let

$$
p: H^{p}(X, \mathbb{Q}(i)) \rightarrow H^{p}(X, \mathbb{Q}(i)) \text { and } q: H^{q}(Y, \mathbb{Q}(j)) \rightarrow H^{q}(Y, \mathbb{Q}(j))
$$

be projectors. Assume that $p$ and $q$ are absolute Hodge. Let $V$ (resp. $W$ ) be the image of $p$ (resp. $q)$, and let

$$
f: H^{p}(X, \mathbb{Q}(i)) \rightarrow H^{q}(Y, \mathbb{Q}(j))
$$

be absolute Hodge. Assume that qfp induces an isomorphism from $V$ to $W$. Then the composition

$$
H^{q}(Y, \mathbb{Q}(j)) \longrightarrow W \stackrel{(q f p)^{-1}}{\longrightarrow} V \longrightarrow H^{p}(X, \mathbb{Q}(i))
$$

is absolute Hodge.

Proof. We need to check that after conjugating by any automorphism of $\mathbb{C}$, the above composition is given by a Hodge class. Since $q, f$ and $p$ are absolute Hodge, we only have to check that this is true for the identity automorphism, which is the case.

This is to compare with Grothendieck's construction of the category of pure motives as a pseudoabelian category, see for instance [3]. 
2.4. Classes coming from the standard conjectures and polarizations. Let $X$ be a smooth projective complex variety of dimension $d$. The cohomology of $X \times X$ carries a number of Hodge classes which are not known to be algebraic. The standard conjectures, as stated in 21, predict that the Künneth components of the diagonal and the inverse of the Lefschetz isomorphism are algebraic. A proof of those would have a lot of consequences in the theory of pure motives. Let us prove that they are absolute Hodge classes. More generally - and informally - any cohomology class obtained from absolute Hodge classes by canonical rational constructions can be proved to be absolute Hodge.

First, let $\Delta$ be the diagonal of $X \times X$. It is an algebraic cycle of codimension $d$ in $X \times X$, hence it has a cohomology class $[\Delta]$ in $H^{2 d}(X \times X, \mathbb{Q}(d))$. By the Künneth formula, we have a canonical isomorphism of Hodge structures

$$
H^{2 d}(X \times X, \mathbb{Q}) \simeq \bigoplus_{i=0}^{2 d} H^{i}(X, \mathbb{Q}) \otimes H^{2 d-i}(X, \mathbb{Q}),
$$

hence projections $H^{2 d}(X \times X, \mathbb{Q}) \rightarrow H^{i}(X, \mathbb{Q}) \otimes H^{2 d-i}(X, \mathbb{Q})$. Let $\pi_{i}$ be the component of $[\Delta]$ in $H^{i}(X, \mathbb{Q}) \otimes H^{2 d-i}(X, \mathbb{Q})(d) \subset H^{2 d}(X \times X, \mathbb{Q})(d)$. The cohomology classes $\pi_{i}$ are the called the Künneth components of the diagonal.

Proposition 18. The Künneth components of the diagonal are absolute Hodge cycles.

Proof. Clearly the $\pi_{i}$ are Hodge classes. Let $\sigma$ be an automorphism of $\mathbb{C}$. Denote by $\Delta^{\sigma}$ the diagonal of $X^{\sigma} \times X^{\sigma}=(X \times X)^{\sigma}$, and by $\pi_{i}^{\sigma}$ the Künneth components of $\Delta^{\sigma}$. Those are also Hodge classes.

Let $\pi_{i, d R}$ (resp. $\left(\pi_{i}^{\sigma}\right)_{d R}$ ) denote the images of the $\pi_{i}$ (resp. $\pi_{i}^{\sigma}$ ) in the de Rham cohomology of $X \times X$ (resp. $\left.X^{\sigma} \times X^{\sigma}\right)$. The Künneth formula holds for de Rham cohomology and is compatible with the comparison isomorphism between de Rham and singular cohomology. It follows that

$$
\left(\pi_{i}^{\sigma}\right)_{d R}=\left(\pi_{i, d R}\right)^{\sigma} .
$$

Since the $\left(\pi_{i}^{\sigma}\right)_{d R}$ are Hodge classes, the conjugates of $\pi_{i, d R}$ are, which concludes the proof.

Fix an embedding of $X$ into a projective space, and let $h \in H^{2}(X, \mathbb{Q}(2))$ be the cohomology class of a hyperplane section. The hard Lefschetz theorem states that for all $i \leq d$, the morphism

$$
L^{d-i}=\cup h^{d-i}: H^{i}(X, \mathbb{Q}) \rightarrow H^{2 d-i}(X, \mathbb{Q}(d-i)), x \mapsto x \cup \xi^{d-i}
$$

is an isomorphism.

Proposition 19. The inverse $f_{i}: H^{2 d-i}(X, \mathbb{Q}(d-i)) \rightarrow H^{i}(X, \mathbb{Q})$ of the Lefschetz isomorphism is absolute Hodge.

Proof. This an immediate consequence of Proposition 16

As an immediate corollary, we get the following result.

Corollary 20. Let $i$ be an integer such that $2 i \leq d$. An element $x \in H^{2 i}(X, \mathbb{Q})$ is an absolute Hodge class if and only if $x \cup \xi^{d-2 i} \in H^{2 d-2 i}(X, \mathbb{Q}(d-2 i))$ is an absolute Hodge class.

Using the preceding results, one introduce polarized Hodge structures in the setting of absolute Hodge classes. Let us start with an easy lemma. 
Lemma 21. Let $X$ be a smooth projective complex variety of dimension $d$, and let $h \in H^{2}(X, \mathbb{Q}(1))$ be the cohomology class of a hyperplane section. Let $L$ denote the operator given by cup-product with $\xi$. Let $i$ be an integer. In the Lefschetz decomposition

$$
H^{i}(X, \mathbb{Q})=\bigoplus_{j \geq 0} L^{j} H^{i-2 j}(X, \mathbb{Q})_{\text {prim }}
$$

of the cohomology of $X$ into primitive parts, the projection of $H^{i}(X, \mathbb{Q})$ onto $L^{j} H^{i-2 j}(X, \mathbb{Q})_{\text {prim }} \hookrightarrow$ $H^{i}(X, \mathbb{Q})$ is given by an absolute Hodge class.

Proof. By induction, it is enough to prove that the projection of $H^{i}(X, \mathbb{Q})$ onto $L H^{i-2}(X, \mathbb{Q})$ is given by an absolute Hodge class. While this could be proved by an argument of Galois equivariance as before, consider the composition

$$
L \circ f_{i} \circ L^{d-i+1}: H^{i}(X, \mathbb{Q}) \rightarrow H^{i}(X, \mathbb{Q})
$$

where $f_{i}: H^{2 d-i}(X, \mathbb{Q}) \rightarrow H^{i}(X, \mathbb{Q})$ is the inverse of the Lefschetz operator. It is the desired projection since $H^{k}(S, \mathbb{Q})_{\text {prim }}$ is the kernel of $L^{d-i+1}$ in $H^{i}(S, \mathbb{Q})$.

This allows for the following result, which shows that the Hodge structures on the cohomology of smooth projective varieties can be polarized by absolute Hodge classes.

Proposition 22. Let $X$ be a smooth projective complex variety and $k$ be an integer. There exists a pairing

$$
Q: H^{k}(X, \mathbb{Q}) \otimes H^{k}(X, \mathbb{Q}) \rightarrow \mathbb{Q}(-k)
$$

which is given by an absolute Hodge class and turns $H^{k}(X, \mathbb{Q})$ into a polarized Hodge structure.

Proof. Let $d$ be the dimension of $X$. By the hard Lefschetz theorem, we can assume $k \leq d$. Let $H$ be an ample line bundle on $X$ with first Chern class $h \in H^{2}(X, \mathbb{Q}(1)$, and let $L$ be the endomorphism of the cohomology of $X$ given by the cup-product with $h$. Consider the Lefschetz decomposition

$$
H^{k}(X, \mathbb{Q})=\bigoplus_{i \geq 0} L^{i} H^{k-2 i}(X, \mathbb{Q})_{\text {prim }}
$$

of $H^{k}(X, \mathbb{Q})$ into primitive parts. Let $s$ be the linear automorphism of $H^{k}(X, \mathbb{Q})$ which is given by multiplication by $(-1)^{i}$ on $L^{i} H^{k-2 i}(X, \mathbb{Q})_{\text {prim }}$.

By the Hodge index theorem, the pairing

$$
H^{k}(X, \mathbb{Q}) \otimes H^{k}(X, \mathbb{Q}) \rightarrow \mathbb{Q}(1), \alpha \otimes \beta \mapsto \int_{X} \alpha \cup L^{d-k}(s(\beta))
$$

turns $H^{2 p}(\overline{\mathcal{X}}, \mathbb{Q})$ into a polarized Hodge structure.

By Lemma 21, the projections of $H^{2 p}(X, \mathbb{Q})$ onto the factors $L^{i} H^{2 p-2 i}(X, \mathbb{Q})_{\text {prim }}$ are given by absolute Hodge classes. It follows that the morphism $s$ is given by an absolute Hodge class.

Since cup-product is given by an absolute Hodge class, see 2.3. and $L$ is induced by an algebraic correspondence, it follows that the pairing $Q$ is given by an absolute Hodge class, which concludes the proof of the proposition.

Proposition 23. Let $X$ and $Y$ be smooth projective complex varieties, and let

$$
f: H^{p}(X, \mathbb{Q}(i)) \rightarrow H^{q}(Y, \mathbb{Q}(j))
$$

be a morphism of Hodge structures. Fix polarizations on the cohomology groups of $X$ and $Y$ given by absolute Hodge classes. Then the orthogonal projection of $H^{p}(X, \mathbb{Q}(i))$ onto Ker $f$ and the orthogonal projection of $H^{q}(Y, \mathbb{Q}(j))$ onto $\left.\operatorname{Im} f\right)$ are given by absolute Hodge classes. 
Proof. The proof of this result is a formal consequence of the existence of polarizations by absolute Hodge classes. It is easy to prove that the projections we consider are absolute using an argument of Galois equivariance as in the preceding paragraph. Let us however give an alternate proof from linear algebra. The abstract argument corresponding to this proof can be found in 1], section 3 . We will only prove that the orthogonal projection of $H^{p}(X, \mathbb{Q}(i))$ onto $\operatorname{Ker} f$ is absolute Hodge, the other statement being a consequence via Poincaré duality.

For ease of notation, we will not write down Tate twists. Those can be recovered by weight considerations. By Poincaré duality, the polarization on $H^{p}(X, \mathbb{Q})$ induces an isomorphism

$$
\phi: H^{p}(X, \mathbb{Q}) \rightarrow H^{2 d-p}(X, \mathbb{Q})
$$

where $d$ is the dimension of $X$, which is absolute Hodge since the polarization is. Similarly, the polarization on $H^{q}(Y, \mathbb{Q})$ induces a morphism

$$
\psi: H^{q}(Y, \mathbb{Q}) \rightarrow H^{2 d^{\prime}-q}(Y, \mathbb{Q})
$$

where $d^{\prime}$ is the dimension of $Y$, which is given by an absolute Hodge class.

Consider the following diagram, which does not commute

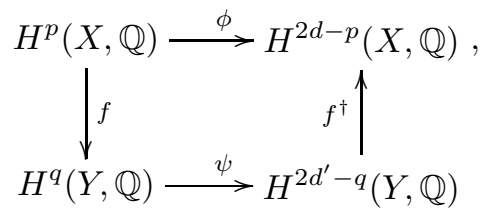

and consider the morphism

$$
h: H^{p}(X, \mathbb{Q}) \rightarrow H^{p}(X, \mathbb{Q}), x \mapsto\left(\phi^{-1} \circ f^{\dagger} \circ \psi \circ f\right)(x) .
$$

Since all the morphisms in the diagram above are given by absolute Hodge classes, $h$ is. Let us compute the kernel and the image of $h$.

Let $x \in H^{p}(X, \mathbb{Q})$. We have $h(x)=0$ if and only if $f^{\dagger} \psi f(x)=0$, which means that for all $y$ in $H^{p}(X, \mathbb{Q})$,

$$
f^{\dagger} \psi f(x) \cup y=0,
$$

that is, since $f$ and $f^{\dagger}$ are transpose of each other :

$$
\psi f(x) \cup f(y)=0,
$$

which exactly means that $f(x)$ is orthogonal to $f\left(H^{p}(X, \mathbb{Q})\right)$ with respect to the polarization of $H^{q}(Y, \mathbb{Q})$. Now the space $f\left(H^{p}(X, \mathbb{Q})\right)$ is a Hodge substructure of the polarized Hodge structure $H^{q}(Y, \mathbb{Q})$. As such, it does not contain any nonzero totally isotropic element. This implies that $f(x)=0$ and shows that

$$
\operatorname{Ker} h=\operatorname{Ker} f \text {. }
$$

Since $f$ and $f^{\dagger}$ are transpose of each other, the image of $h$ is clearly contained in $(\operatorname{Ker} f)^{\perp}$. Considering the rank of $h$, this readily shows that

$$
\operatorname{Im} h=(\operatorname{Ker} f)^{\perp} .
$$

The two subspaces $\operatorname{Ker} h=\operatorname{Ker} f$ and $\operatorname{Im} h=(\operatorname{Ker} f)^{\perp}$ of $H^{p}(X, \mathbb{Q})$ are in direct sum. By standard linear algebra, it follows that the orthogonal projection $p$ of $H^{p}(X, \mathbb{Q})$ onto $(\operatorname{Ker} f)^{\perp}$ is a polynomial in $h$ with rational coefficients. Since $h$ is given by an absolute Hodge class, so is $p$, as well as Id $-p$, which is the orthogonal projection onto Ker $f$. 
Corollary 24. Let $X$ and $Y$ be two smooth projective complex varieties, and let

$$
f: H^{p}(X, \mathbb{Q}(i)) \rightarrow H^{q}(Y, \mathbb{Q}(j))
$$

be a morphism given by an absolute Hodge class. Let $\alpha$ be an absolute Hodge class in the image of $f$. Then there exists an absolute Hodge class $\beta \in H^{p}(X, \mathbb{Q}(i))$ such that $f(\beta)=\alpha$.

Proof. By Proposition 23, the orthogonal projection of $H^{p}(X, \mathbb{Q}(i))$ onto $(\operatorname{Ker} f)^{\perp}$ and the orthogonal projection of $H^{q}(Y, \mathbb{Q}(j))$ onto $\left.\operatorname{Im} f\right)$ are given by absolute Hodge classes. Now Proposition 17 shows that the composition

$$
H^{q}(Y, \mathbb{Q}(j)) \longrightarrow \operatorname{Im} f \stackrel{(q f p)^{-1}}{\longrightarrow}(\operatorname{Ker} f)^{\perp \subset} \longrightarrow H^{p}(X, \mathbb{Q}(i))
$$

is absolute Hodge. As such, it sends $\alpha$ to an absolute Hodge class $\beta$. Since $\alpha$ belongs to the image of $f$, we have $f(\beta)=\alpha$.

The results we proved in this paragraph and the preceding one are the ones needed to construct a category of motives for absolute Hodge cycles and prove it is a semi-simple abelian category. This is done in [17. In that sense, absolute Hodge classes provide a way to work with an unconditional theory of motives, to quote André.

We actually proved more. Indeed, while the explicit proofs we gave of Proposition 21 and Proposition 23 might seem a little longer than what would be needed, they provide the cohomology classes we need using only classes coming from the standard conjectures. This is the basis for André's notion of motivated cycles described in [1. This paper shows that a lot of the results we obtain here about the existence of some absolute Hodge classes can be actually strengthened to motivated cycles. In particular, the algebraicity of the absolute Hodge classes we consider, which is a consequence of the Hodge conjecture, is most of the time implied by the standard conjectures.

2.5. Absolute Hodge classes and the Hodge conjecture. Let $X$ be a smooth projective complex variety. We proved earlier that the cohomology class of an algebraic cycle in $X$ is absolute Hodge. This remark allows us to split the Hodge conjecture in the two following conjectures.

Conjecture 25. Let $X$ be a smooth projective complex variety. Let $p$ be a nonnegative integer, and let $\alpha$ be an element of $H^{2 p}(X, \mathbb{Q}(p))$. Then $\alpha$ is a Hodge class if and only if it is an absolute Hodge class.

Conjecture 26. Let $X$ be a smooth projective complex variety. For any nonnegative integer $p$, the subspace of degree $p$ absolute Hodge classes is generated over $\mathbb{Q}$ by the cohomology classes of codimension $p$ subvarieties of $X$.

Those statements are a tentative answer to the problem we raised in paragraph 2.1. Indeed, while those two conjectures together imply the Hodge conjecture, neither of them makes sense in the setting of Kähler manifolds. The main feature of an algebraic variety $X$ we use here, which is not shared by general compact Kähler manifolds, is that such an $X$ always can be realized as the generic fiber of a morphism of algebraic varieties defined over a number field. We will go back to this point of view in the next section when studying the relationship between Hodge loci and absolute Hodge classes. The fact that we can use varieties over number fields is the reason why Galois actions can enter the picture in the algebraic setting.

This situation however does not happen outside the algebraic world, as automorphisms of $\mathbb{C}$ other than the identity and complex conjugation are very discontinuous - e.g., they are not measurable. This makes it impossible to give a meaning to the conjugate of a complex manifold by an automorphism of $\mathbb{C}$. 
Even for algebraic varieties, the fact that automorphisms of $\mathbb{C}$ are highly discontinuous appears. Let $\sigma$ be an automorphism of $\mathbb{C}$, and let $X$ be a smooth projective complex variety. Equation (4) induces a $\sigma$-linear isomorphism

$$
\left(\sigma^{-1}\right)^{*}: H^{*}\left(X^{a n}, \mathbb{C}\right) \rightarrow H^{*}\left(\left(X^{\sigma}\right)^{a n}, \mathbb{C}\right)
$$

between the singular cohomology with complex coefficients of the complex manifolds underlying $X$ and $X^{\sigma}$. Conjecture 25 means that Hodge classes in $H^{*}\left(X^{a n}, \mathbb{C}\right)$ should map to Hodge classes in $H^{*}\left(\left(X^{\sigma}\right)^{a n}, \mathbb{C}\right)$. In particular, they should map to elements of the rational subspace $H^{*}\left(\left(X^{\sigma}\right)^{a n}, \mathbb{Q}\right)$.

However, it is not to be expected that $\left(\sigma^{-1}\right)^{*}$ maps $H^{*}\left(X^{a n}, \mathbb{Q}\right)$ to $H^{*}\left(\left(X^{\sigma}\right)^{a n}, \mathbb{Q}\right)$. It can even happen that the two algebras $H^{*}\left(X^{a n}, \mathbb{Q}\right)$ and $H^{*}\left(\left(X^{\sigma}\right)^{a n}, \mathbb{Q}\right)$ are not isomorphic, see 11. This implies in particular that the complex varieties $X^{a n}$ and $\left(X^{\sigma}\right)^{a n}$ need not be homeomorphic, while the schemes $X$ and $X^{\sigma}$ are. This also shows that singular cohomology with rational algebraic coefficients can not be defined algebraically 2 .

The main goal of these notes is to discuss Conjecture 25. We will give a number of example of absolute Hodge classes which are not known to be algebraic, and describe some applications. While Conjecture 26 seems to be very open at the time, we can make two remarks about it.

Let us first state a result which might stand as a motivation for the statement of this conjecture. We mentioned above that conjugation by an automorphism of $\mathbb{C}$ does not in general preserve singular cohomology with rational coefficients, but it does preserve absolute Hodge classes by definition.

Let $X$ be a smooth projective complex variety. The singular cohomology with rational coefficients of the underlying complex manifold $X^{a n}$ is spanned by the cohomology classes of images of real submanifolds of $X^{a n}$. The next result, see [32], Lemma 28 for a related statement, shows that among closed subsets of $X^{a n}$, algebraic subvarieties are the only one that remain closed after conjugation by an automorphism of $\mathbb{C}$.

Recall that if $\sigma$ is an automorphism of $\mathbb{C}$, we have an isomorphism of schemes

$$
\sigma: X \rightarrow X^{\sigma} \text {. }
$$

It sends complex points of $X$ to complex points of $X^{\sigma}$.

Proposition 27. Let $X$ be a complex variety, and let $F$ be a closed subset of $X^{\text {an }}$. Assume that for any automorphism $\sigma$ of $\mathbb{C}$, the subset

$$
\sigma(F) \subset X^{\sigma}(\mathbb{C})
$$

is closed in $\left(X^{\sigma}\right)^{a n}$. Then $F$ is a countable union of algebraic subvarieties of $X$.

Proof. Using induction on the dimension of $X$, we can assume that $F$ is not contained in a countable union of proper subvarieties of $X$. We want to prove that $F=X$. Using a finite map from $X$ to a projective space, we can assume that $X=\mathbb{A}_{\mathbb{C}}^{n}$. Our hypothesis is thus that $F$ is a closed subset of $\mathbb{C}^{n}$ which is not contained in a countable union of proper subvarieties of $\mathbb{C}^{n}$, such that for any automorphism of $\mathbb{C}, \sigma(F)=\left\{\left(\sigma\left(x_{1}\right), \ldots, \sigma\left(x_{n}\right)\right),\left(x_{1}, \ldots, x_{n}\right) \in \mathbb{C}^{n}\right\}$ is closed in $\mathbb{C}^{n}$. We will use an elementary lemma.

Lemma 28. Let $k$ be a countable subfield of $\mathbb{C}$. There exists a point $\left(x_{1}, \ldots, x_{n}\right)$ in $F$ such that the complex numbers $\left(x_{1}, \ldots, x_{n}\right)$ are algebraically equivalent over $k$.

\footnotetext{
${ }^{2}$ While the isomorphism we gave between the algebras $H^{*}\left(X^{a n}, \mathbb{C}\right)$ and $H^{*}\left(\left(X^{\sigma}\right)^{a n}, \mathbb{C}\right)$ is not $\mathbb{C}$-linear, it is possible to show using étale cohomology that there exists a $\mathbb{C}$-linear isomorphism between those two algebras, depending on an embedding of $\mathbb{Q}_{l}$ into $\mathbb{C}$.
} 
Proof. Since $k$ is countable, there exists only a countable number of algebraic subvarieties of $\mathbb{C}^{n}$ defined over $k$. By our assumption on $F$, there exists a point of $F$ which does not lie in any proper algebraic variety defined over $k$. Such a point has coordinates which are algebraically independent over $k$.

Using the preceding lemma and induction, we can find a sequence of points

$$
p_{i}=\left(x_{1}^{i}, \ldots, x_{n}^{i}\right) \in F
$$

such that the $\left(x_{j}^{i}\right)_{i \in \mathbb{N}, j \leq n}$ are algebraically independent over $\mathbb{Q}$. Now let $\left(y_{j}^{i}\right)_{i \in \mathbb{N}, j \leq n}$ be a sequence of points in $\mathbb{C}^{n}$ such that $\left\{\left(y_{1}^{i}, \ldots, y_{n}^{i}\right), i \in \mathbb{N}\right\}$ is dense in $\mathbb{C}^{n}$. We can find an automorphism $\sigma$ of $\mathbb{C}$ mapping $x_{j}^{i}$ to $y_{j}^{i}$ for all $i, j$. The closed subset of $\mathbb{C}^{n} \sigma(F)$ contains a dense subset of $\mathbb{C}^{n}$, hence $\sigma(F)=\mathbb{C}^{n}$. This shows that $F=\mathbb{C}^{n}$ and concludes the proof.

As an immediate consequence, we get the following.

Proposition 29. Let $X$ be a complex projective variety, and let $F$ be a closed subset of $X^{\text {an }}$. Assume that for any automorphism $\sigma$ of $\mathbb{C}$, the subset

$$
\sigma(F) \subset X^{\sigma}(\mathbb{C})
$$

is closed in $\left(X^{\sigma}\right)^{a n}$. Then $F$ is an algebraic subvariety of $X$.

Given that absolute Hodge classes are classes in the singular cohomology groups that are, in some sense, Galois equivariant $\sqrt{3}$ and that by the preceding result, algebraic subvarieties are the only closed subsets with a good behavior with respect to the Galois action, this might serve as a motivation for Conjecture 26.

Another, more precise, reason that explains why Conjecture 26 might be more tractable than the Hodge conjecture is given by the work of André around motivated cycles in [1]. Through motivic considerations, André does indeed show that for most of the absolute Hodge classes we know, Conjecture 26 is actually a consequence of the standard conjectures, which, at least in characteristic zero, seem considerably weaker than the Hodge conjecture.

While we won't prove such results, it is to be noted that the proofs we gave in Paragraphs 2.3 and 2.4 were given so as to imply André's results for the absolute Hodge classes we will consider. The interested reader should have no problem filling the gaps.

In the following sections, we will not use the notation $X^{a n}$ for the complex manifold underlying a complex variety $X$ anymore, but rather, by an abuse of notation, use $X$ to refer to both objects. The context will hopefully help the reader avoid any confusion.

\section{Absolute Hodge classes in families}

This section deals with the behavior of absolute Hodge classes under deformation. We will focus on consequences of the algebraicity of Hodge bundles. We prove Deligne's principle B, which states that absolute Hodge classes are preserved by parallel transport, and discuss the link between Hodge loci and absolute Hodge classes as in 31. The survey 33. contains a beautiful account of similar results.

We only work here with projective families. The quasi-projective case could be dealt with and provide similar results, see for instance [12.

\footnotetext{
${ }^{3}$ See [16], Question 2.4, where the questions of whether those are the only ones is raised.
} 
3.1. The variational Hodge conjecture and the global invariant cycle theorem. Before stating Deligne's Principle B in [16, let us explain a variant of the Hodge conjecture.

Let $S$ be a smooth connected quasi-projective variety, and let $\pi: \mathcal{X} \rightarrow S$ be a smooth projective morphism. Let 0 be a complex point of $S$, and, for some integer $p$ let $\alpha$ be a cohomology class in $H^{2 p}\left(\mathcal{X}_{0}, \mathbb{Q}(p)\right)$. Assume that $\alpha$ is the cohomology class of some codimension $p$ algebraic cycle $Z_{0}$, and that $\alpha$ extends as a section $\widetilde{\alpha}$ of the local system $R^{2 p} \pi_{*} \mathbb{Q}(p)$ on $S^{\mathbb{4}}$.

In 20], footnote 13, Grothendieck makes the following conjecture.

Conjecture 30. (Variational Hodge conjecture) For any complex point $s$ of $S$, the class $\widetilde{\alpha}_{s}$ is the cohomology class of an algebraic cycle.

Using the Gauss-Manin connection and the isomorphism between de Rham and singular cohomology, we can formulate an alternative version of the variational Hodge conjecture in de Rham cohomology. For this, keeping the notations as above, we have a coherent sheaf $\mathcal{H}^{2 p}=\mathbb{R}^{2 p} \pi_{*} \Omega_{\mathcal{X} / S}^{\bullet}$ which computes the relative de Rham cohomology of $\mathcal{X}$ over $S$. As we saw earlier, it is endowed with a canonical connection, the Gauss-Manin connection $\nabla$.

Conjecture 31. (Variational Hodge conjecture for de Rham cohomology) Let $\beta$ be a cohomology class in $H^{2 p}\left(\mathcal{X}_{0} / \mathbb{C}\right)$. Assume that $\beta$ is the cohomology class of some codimension $p$ algebraic cycle $Z_{0}$, and that $\beta$ extends as a section $\widetilde{\beta}$ of the coherent sheaf $\mathcal{H}^{2 p}=\mathbb{R}^{2 p} \pi_{*} \Omega_{\mathcal{X} / S}$ such that $\widetilde{\beta}$ is flat for the Gauss-Manin connection. The variational Hodge conjecture states that for any complex point $s$ of $S$, the class $\widetilde{\beta}_{s}$ is the cohomology class of an algebraic cycle.

Proposition 32. Conjecture 30 and 31 are equivalent.

Proof. The de Rham comparison isomorphism between singular and de Rham cohomology in a relative context takes the form of a canonical isomorphism

$$
\mathbb{R}^{2 p} \pi_{*} \Omega_{\mathcal{X} / S}^{\bullet} \simeq R^{2 p} \pi_{*} \mathbb{Q}(p) \otimes_{\mathbb{Q}} \mathcal{O}_{S}
$$

Note that this formula is not one from algebraic geometry. Indeed, the sheaf $\mathcal{O}_{S}$ denotes here the sheaf of holomorphic functions on the complex manifold $S$. The derived functor $\mathbb{R}^{2 p} \pi_{*}$ on the left is a functor between categories of complexes of holomorphic coherent sheaves, while the one on the right is computed for sheaves with the usual complex topology. The Gauss-Manin connection is the connection on $\mathbb{R}^{2 p} \pi_{*} \Omega_{\mathcal{X} / S}^{\bullet}$ for which the local system $R^{2 p} \pi_{*} \mathbb{Q}(p)$ is constant. As we saw earlier, the locally free sheaf $\mathbb{R}^{2 p} \pi_{*} \Omega_{\mathcal{X} / S}$ is algebraic, as well as the Gauss-Manin connection.

Given $\beta$ a cohomology class in the de Rham cohomology group $H^{2 p}\left(\mathcal{X}_{0} / \mathbb{C}\right)$ as above, we know that $\beta$ belongs to the rational subspace $H^{2 p}\left(\mathcal{X}_{0}, \mathbb{Q}(p)\right)$ because it is the cohomology class of an algebraic cycle. Furthermore, since $\widetilde{\beta}$ is flat for the Gauss-Manin connection and is rational at one point, it corresponds to a section of the local system $R^{2 p} \pi_{*} \mathbb{Q}(p)$ under the comparison isomorphism above. This shows that Conjecture 30 implies Conjecture 31

On the other hand, sections of the local system $R^{2 p} \pi_{*} \mathbb{Q}(p)$ induce flat algebraic sections of the coherent sheaf $\mathbb{R}^{2 p} \pi_{*} \Omega_{\mathcal{X} / S}$. By definition of the Gauss-Manin connection, they do induce flat holomorphic sections, and we have to show that those are algebraic. This is a consequence of the following important result, which is due to Deligne.

\footnotetext{
${ }^{4}$ Once again, the use of a Tate twist might seem superfluous. We write it down in order to emphasize the variation of Hodge structures that this local system underlies, as well as the factor $(2 \pi i)^{-p}$ that appears in the comparison isomorphism with de Rham cohomology.
} 
Theorem 33. (Global invariant cycle theorem) Let $\pi: \mathcal{X} \rightarrow S$ be a smooth projective morphism of quasi-projective complex varieties, and let $i: \mathcal{X} \hookrightarrow \overline{\mathcal{X}}$ be a smooth compactification of $\mathcal{X}$. Let 0 be complex point of $S$, and let $\pi_{1}(S, 0)$ be the fundamental group of $S$. For any integer $k$, the space of monodromy-invariant classes of degree $k$

$$
H^{k}\left(\mathcal{X}_{0}, \mathbb{Q}\right)^{\pi_{1}(S, 0)}
$$

is equal to the image of the restriction map

$$
i_{0}^{*}: H^{k}(\overline{\mathcal{X}}, \mathbb{Q}) \rightarrow H^{k}\left(\mathcal{X}_{0}, \mathbb{Q}\right),
$$

where $i_{0}$ is the inclusion of $\mathcal{X}_{0}$ in $\overline{\mathcal{X}}$.

In the theorem, the monodromy action is the action of the fundamental group $\pi_{1}(S, 0)$ on the cohomology groups of the fiber $\mathcal{X}_{0}$. Note that the theorem implies that the space $H^{k}\left(\mathcal{X}_{0}, \mathbb{Q}\right)^{\pi_{1}(S, 0)}$ is a sub-Hodge structure of $H^{k}\left(\mathcal{X}_{0}, \mathbb{Q}\right)$. However, the fundamental group of $S$ does not in general act by automorphisms of Hodge structures.

The global invariant cycle theorem implies the algebraicity of flat holomorphic sections of the vecotr bundle $\mathbb{R}^{2 p} \pi_{*} \Omega_{\mathcal{X} / S}^{\bullet}$ as follows. Let $\widetilde{\beta}$ be such a section, and keep the notation of the theorem. By definition of the Gauss-Manin connection, $\widetilde{\beta}$ corresponds to a section of the local system $R^{2 p} \pi_{*} \mathbb{C}(p)$ under the isomorphism 5 , that is, to a monodromy-invariant class in $H^{2 p}\left(\mathcal{X}_{0}, \mathbb{C}(p)\right)$. The global invariant cycle theorem shows, using the comparison theorem between singular and de Rham cohomology on $\overline{\mathcal{X}}$, that $\widetilde{\beta}$ comes from a de Rham cohomology class $b$ in $H^{2 p}(\overline{\mathcal{X}} / \mathbb{C})$. As such, it is algebraic.

The preceding remarks readily show the equivalence of the two versions of the variational Hodge conjecture.

The next proposition shows that the variational Hodge conjecture is actually a part of the Hodge conjecture. This fact is a consequence of the global invariant cycle theorem. The following proof will be rewritten in the next paragraph to give results on absolute Hodge cycles.

Proposition 34. Let $S$ be a smooth connected quasi-projective variety, and let $\pi: \mathcal{X} \rightarrow S$ be a smooth projective morphism. Let 0 be a complex point of $S$, and let $p$ be an integer.

(1) Let $\alpha$ be a cohomology class in $H^{2 p}\left(\mathcal{X}_{0}, \mathbb{Q}(p)\right)$. Assume that $\alpha$ is a Hodge class and that $\alpha$ extends as a section $\widetilde{\alpha}$ of the local system $R^{2 p} \pi_{*} \mathbb{Q}(p)$ on $S$. Then for any complex point $s$ of $S$, the classes $\widetilde{\alpha}_{s}$ is a Hodge class.

(2) Let $\beta$ be a cohomology class in $H^{2 p}\left(\mathcal{X}_{0} / \mathbb{C}\right)$. Assume that $\beta$ is a Hodge class and that $\beta$ extends as a section $\widetilde{\beta}$ of the coherent sheaf $R^{2 p} \pi_{*} \Omega_{\mathcal{X} / S}$ such that $\widetilde{\beta}$ is flat for the GaussManin connection. Then for any complex point s of $S$, the classes $\widetilde{\beta}_{s}$ is a Hodge class.

As an immediate corollary, we get the following.

Corollary 35. The Hodge conjecture implies the variational Hodge conjecture.

Proof. The two statements of the proposition are equivalent by the same arguments as in Proposition 32. Let us keep the notations as above. We want to prove that for any complex point $s$ of $S$, the class $\widetilde{\alpha}_{s}$ is a Hodge class. Let us show how this is a consequence of the global invariant cycle theorem. This is a simple consequence of Corollary 24 in the - easier - context of Hodge classes. Let us prove the result from scratch. 
As in Proposition 22, we can find a pairing

$$
H^{2 p}(\overline{\mathcal{X}}, \mathbb{Q}) \otimes H^{2 p}(\overline{\mathcal{X}}, \mathbb{Q}) \rightarrow \mathbb{Q}(1)
$$

which turns $H^{2 p}(\overline{\mathcal{X}}, \mathbb{Q})$ into a polarized Hodge structure.

Let $i: \mathcal{X} \hookrightarrow \overline{\mathcal{X}}$ be a smooth compactification of $\mathcal{X}$, and let $i_{0}$ be the inclusion of $\mathcal{X}_{0}$ in $\overline{\mathcal{X}}$.

By the global invariant cycle theorem, the morphism

$$
i_{0}^{*}: H^{2 p}(\overline{\mathcal{X}}, \mathbb{Q}) \rightarrow H^{2 p}\left(\mathcal{X}_{0}, \mathbb{Q}\right)^{\pi_{1}(S, 0)}
$$

is surjective. It restricts to an isomorphism of Hodge structures

$$
i_{0}^{*}:\left(\operatorname{Ker} i_{0}^{*}\right)^{\perp} \rightarrow H^{2 p}\left(\mathcal{X}_{0}, \mathbb{Q}\right)^{\pi_{1}(S, 0)},
$$

hence a Hodge class $a \in\left(\operatorname{Ker}_{0}^{*}\right)^{\perp} \subset H^{2 p}(\overline{\mathcal{X}}, \mathbb{Q})$ mapping to $\alpha$. Indeed, saying that $\alpha$ extends to a global section of the local system $R^{2 p} \pi_{*} \mathbb{Q}(p)$ exactly means that $\alpha$ is monodromy-invariant, and $\alpha$ is a Hodge class since it is a cohomology class of an algebraic cycle.

Now let $i_{s}$ be the inclusion of $\mathcal{X}_{s}$ in $\overline{\mathcal{X}}$. Since $S$ is connected, we have $\widetilde{\alpha}_{s}=i_{s}^{*}(a)$, which shows that $\widetilde{\alpha}_{s}$ is a Hodge class, and that the Hodge conjecture implies it is the cohomology class of an algebraic cycle.

It is an important fact that the variational Hodge conjecture is a purely algebraic statement. Indeed, we saw earlier that both relative de Rham cohomology and the Gauss-Manin connection can be defined algebraically. This is to be compared to the above discussion of the transcendental aspect of the Hodge conjecture, where one cannot avoid to use singular cohomology, which cannot be defined in a purely algebraic fashion as it does depend on the topology of $\mathbb{C}$.

Very little seems to be known about the variational Hodge conjecture, see however [5].

3.2. Deligne's Principle B. In this paragraph, we state and prove the so-called Principle B for absolute Hodge cycles, which is due to Deligne. It shows that the variational Hodge conjecture is true if one replaces algebraic cohomology classes by absolute Hodge classes.

Theorem 36. (Principle B) Let $S$ be a smooth connected quasi-projective variety, and let $\pi: \mathcal{X} \rightarrow S$ be a smooth projective morphism. Let 0 be a complex point of $S$, and, for some integer $p$ let $\alpha$ be a cohomology class in $H^{2 p}\left(\mathcal{X}_{0}, \mathbb{Q}(p)\right)$. Assume that $\alpha$ is an absolute Hodge class and that $\alpha$ extends as a section $\widetilde{\alpha}$ of the local system $R^{2 p} \pi_{*} \mathbb{Q}(p)$ on $S$. Then for any complex point $s$ of $S$, the class $\widetilde{\alpha}_{s}$ is absolute Hodge.

As in Proposition 32, this is equivalent to the following rephrasing.

Theorem 37. (Principle $B$ for de Rham cohomology) Let $S$ be a smooth connected quasi-projective variety, and let $\pi: \mathcal{X} \rightarrow S$ be a smooth projective morphism. Let 0 be a complex point of $S$, and, for some integer $p$ let $\beta$ be a cohomology class in $H^{2 p}\left(\mathcal{X}_{0} / \mathbb{C}\right)$. Assume that $\beta$ is an absolute Hodge class and that $\beta$ extends as a flat section $\widetilde{\beta}$ of the locally free sheaf $\mathcal{H}^{2 p}=\mathbb{R}^{2 p} \pi_{*} \Omega_{\mathcal{X} / S}$ endowed with the Gauss-Manin connection. Then for any complex point s of $S$, the class $\widetilde{\beta}_{s}$ is absolute Hodge.

We will give two different proofs of this result to illustrate the techniques we introduced earlier. Both rely on Proposition 34 and on the global invariant cycle theorem. The first one proves the result as a consequence of the algebraicity of the Hodge bundles and of the Gauss-Manin connection. It is essentially Deligne's proof in [16. The second proof elaborates on polarized Hodge structures and is inspired by André's approach in [1]. 
Proof. We will work with de Rham cohomology. Let $\sigma$ be an automorphism of $\mathbb{C}$. Since $\widetilde{\beta}$ is a global section of the locally free sheaf $\mathcal{H}^{2 p}$, we can form the conjugate section $\widetilde{\beta}^{\sigma}$ of the conjugate sheaf $\left(\mathcal{H}^{2 p}\right)^{\sigma}$ on $S^{\sigma}$. Now as in 2.2, this sheaf identifies with the relative de Rham cohomology of $\mathcal{X}^{\sigma}$ over $S^{\sigma}$.

Fix a complex point $s$ in $S$, the class $\widetilde{\beta}_{s}$ is absolute Hodge. This means that for any such $\sigma$, the class $\widetilde{\beta}_{\sigma(s)}^{\sigma}$ is a Hodge class. Now since $\beta=\widetilde{\beta}_{0}$ is an absolute Hodge class by assumption, $\widetilde{\beta}_{\sigma(0)}^{\sigma}$ is a Hodge class.

Since the construction of the Gauss-Manin connection commutes with base change, the GaussManin connection $\nabla^{\sigma}$ on the relative de Rham cohomology of $\mathcal{X}^{\sigma}$ over $S^{\sigma}$ is the conjugate by $\sigma$ of the Gauss-Manin connection on $\mathcal{H}^{2 p}$.

These remarks allow us to write

$$
\nabla^{\sigma} \widetilde{\beta}^{\sigma}=(\nabla \widetilde{\beta})^{\sigma}=0
$$

since $\widetilde{\beta}$ is flat. This shows that $\widetilde{\beta}^{\sigma}$ is a flat section of the relative de Rham cohomology of $\mathcal{X}^{\sigma}$ over $S^{\sigma}$. Since $\widetilde{\beta}_{\sigma(0)}^{\sigma}$ is a Hodge class, Proposition 34 shows that $\widetilde{\beta}_{\sigma(0)}^{\sigma}$ is a Hodge class, which is what we needed to prove.

Note that while the above proof may seem just a formal computation, it actually uses in an essential way the important fact that both relative de Rham cohomology and the Gauss-Manin connection are algebraic object, which makes it possible to conjugate them by field automorphisms.

Let us give a second proof of Principle B.

Proof. This is a consequence of Corollary 24. Indeed, let $i: \mathcal{X} \hookrightarrow \overline{\mathcal{X}}$ be a smooth compactification of $\mathcal{X}$, and let $i_{0}$ be the inclusion of $\mathcal{X}_{0}$ in $\overline{\mathcal{X}}$.

By the global invariant cycle theorem, the morphism

$$
i_{0}^{*}: H^{2 p}(\overline{\mathcal{X}}, \mathbb{Q}) \rightarrow H^{2 p}\left(\mathcal{X}_{0}, \mathbb{Q}\right)^{\pi_{1}(S, 0)}
$$

is surjective. As a consequence, since $\alpha$ is monodromy-invariant, it belongs to the image of $i_{0}^{*}$. By Corollary 24, we can find an absolute Hodge class $a \in H^{2 p}(\overline{\mathcal{X}}, \mathbb{Q})$ mapping to $\alpha$. Now let $i_{s}$ be the inclusion of $\mathcal{X}_{s}$ in $\overline{\mathcal{X}}$. Since $S$ is connected, we have

$$
\widetilde{\alpha}_{s}=i_{s}^{*}(a),
$$

which shows that $\widetilde{\alpha}_{s}$ is an absolute Hodge class, and concludes the proof.

Note that following the remarks we made around the notion of motivated cycles, this argument could be used to prove that the standard conjectures imply the variational Hodge conjecture, see [1.

Principle B will be one of our main tools in proving that some Hodge classes are absolute. When working with families of varieties, it allows us to work with specific members of the family where algebraicity results might be known. When proving the algebraicity of the Kuga-Satake correspondence between a projective K3 surface and its Kuga-Satake abelian variety, it will make it possible to reduce to the case of Kummer surfaces, while in the proof of Deligne's theorem that Hodge classes on abelian varieties are absolute, it allows for a reduction to the case of abelian varieties with complex multiplication. Its mixed case version is instrumental to the results of [12]. 
3.3. The locus of Hodge classes. In this paragraph, we recall the definitions of the Hodge locus and the locus of Hodge classes associated to a variation of Hodge structures and discuss their relation to the Hodge conjecture. The study of those has been started by Griffiths in 21. References on this subject include [28, chapter 17 and $[33$. To simplify matters, we will only deal with variations of Hodge structures coming from geometry, that is, coming from the cohomology of a family of smooth projective varieties. We will point out statements that generalize to the quasi-projective case.

Let $S$ be a smooth complex quasi-projective variety, and let $\pi: \mathcal{X} \rightarrow S$ be a smooth projective morphism. Let $p$ be an integer. As earlier, consider the Hodge bundles

$$
\mathcal{H}^{2 p}=\mathbb{R}^{2 p} \pi_{*} \Omega_{\mathcal{X} / S}
$$

together with the Hodge filtration

$$
F^{k} \mathcal{H}^{2 p}=\mathbb{R}^{2 p} \pi_{*} \Omega_{\mathcal{X} / S}^{\bullet \geq k}
$$

Those are algebraic vector bundles over $S$, as we saw before. They are endowed with the GaussManin connection

Furthermore, the local system

$$
\nabla: \mathcal{H}^{2 p} \rightarrow \mathcal{H}^{2 p} \otimes \Omega_{\mathcal{X} / S}^{1}
$$

$$
H_{\mathbb{Q}}^{2 p}=R^{2 p} \pi_{*} \mathbb{Q}(p)
$$

injects into $\mathcal{H}^{2 p}$ and is flat with respect to the Gauss-Manin connection.

Let us start with a set-theoretic definition of the locus of Hodge classes.

Definition 38. The locus of Hodge classes in $\mathcal{H}^{2 p}$ is the set of pairs $(\alpha, s), s \in S(\mathbb{C}), \alpha \in \mathcal{H}_{s}^{2 p}$, such that $\alpha$ is a Hodge class, that is, $\alpha \in F^{p} \mathcal{H}_{s}^{2 p}$ and $\alpha \in H_{\mathbb{Q}, s}^{2 p}$.

It turns out that the locus of Hodge classes is the set of complex points of a countable union of analytic subvarieties of $\mathcal{H}^{2 p}$. This can be seen as follows, see the above references for a thorough description. Let $(\alpha, s)$ be in the locus of Hodge classes of $\mathcal{H}^{2 p}$. We want to describe the component of the locus of Hodge classes passing through $(\alpha, s)$ as an analytic variety in a neighborhood of $(\alpha, s)$.

On a neighborhood of $s$, the class $\widetilde{\alpha}$ extends to a flat holomorphic section of $\mathcal{H}^{2 p}$. Now the points $\left(\widetilde{\alpha}_{t}, t\right)$, for $t$ in the neighborhood of $s$, which belong to the locus of Hodge classes are the points of an analytic variety, namely the variety defined by the $\left(\widetilde{\alpha}_{t}, t\right)$ such that $\widetilde{\alpha}_{t}$ vanishes in the holomorphic (and even algebraic) vector bundle $\mathcal{H}^{2 p} / F^{p} \mathcal{H}^{2 p}$.

It follows from this remark that the locus of Hodge classes is a countable union of analytic subvarieties of $\mathcal{H}^{2 p}$. Note that if we were to consider only integer cohomology classes to define the locus of Hodge classes, we would actually get an analytic subvariety. The locus of Hodge classes was introduced in [10. It is of course very much related to the more classical Hodge locus.

Definition 39. The Hodge locus associated to $\mathcal{H}^{2 p}$ is the projection on $S$ of the locus of Hodge classes. It is a countable union of analytic subvarieties of $S$.

Note that the Hodge locus is interesting only when $\mathcal{H}^{2 p}$ has no flat global section of type $(p, p)$. Indeed, if it has, the Hodge locus is $S$ itself. However, in this case, one can always split off any constant variation of Hodge structures for $\mathcal{H}^{2 p}$ and consider the Hodge locus for the remaining variation of Hodge structures.

The reason why we are interested in those loci is the way they are related to the Hodge conjecture. Indeed, one has the following. 
Proposition 40. If the Hodge conjecture is true, then the locus of Hodge classes and the Hodge locus for $\mathcal{H}^{2 p} \rightarrow S$ are countable unions of closed algebraic subsets of $\mathcal{H}^{2 p} \times S$ and $S$ respectively.

Proof. We only have to prove the proposition for the locus of Hodge classes. If the Hodge conjecture is true, the locus of Hodge classes is the locus of cohomology classes of algebraic cycles with rational coefficients. Those algebraic cycles are parametrized by Hilbert schemes for the family $\mathcal{X} / B$. Since those are proper and have countably many connected components, the Hodge locus is a countable union of closed algebraic subsets of $\mathcal{H}^{2 p} \times S$.

This consequence of the Hodge conjecture is now a theorem proved in [10].

Theorem 41. (Cattani, Deligne, Kaplan) With the notations above, the locus of Hodge classes and the Hodge locus for $\mathcal{H}^{2 p} \rightarrow S$ are countable unions of closed algebraic subsets of $\mathcal{H}^{2 p} \times S$ and $S$ respectively.

As before, the preceding discussion can be led in the quasi-projective case. Generalized versions of the Hodge conjecture lead to similar algebraicity predictions, and indeed the corresponding algebraicity result for variations of mixed Hodge structures is proved in [8], after the work of Brosnan-Pearlstein on the zero locus of normal functions in [7].

3.4. Galois action on relative de Rham cohomology. Let $S$ be a smooth irreducible quasiprojective variety over a field $k$, and let $\pi: \mathcal{X} \rightarrow S$ be a smooth projective morphism. Let $p$ be an integer. Consider again the Hodge bundles $\mathcal{H}^{2 p}$ together with the Hodge filtration $F^{k} \mathcal{H}^{2 p}=$ $R^{2 p} \pi_{*} \Omega_{\mathcal{X} / S}^{\bullet}$. Those are defined over $k$.

Let $\alpha$ be a section of $\mathcal{H}^{2 p}$ over $S$. Let $\eta$ be the generic point of $S$. The class $\alpha$ induces a class $\alpha_{\eta}$ in the de Rham cohomology of the generic fiber $\mathcal{X}_{\eta}$ of $\pi$.

Let $\sigma$ be any embedding of $k(S)$ in $\mathbb{C}$ over $k$. The morphism $\sigma$ corresponds to a morphism $\operatorname{Spec}(\mathbb{C}) \rightarrow \eta \rightarrow S$, hence it induces a complex point $s$ of $S_{\mathbb{C}}$. We have an isomorphism

$$
\mathcal{X}_{\eta} \times_{k(S)} \mathbb{C} \simeq \mathcal{X}_{\mathbb{C}, s}
$$

and the cohomology class $\alpha_{\eta}$ pulls back to a class $\alpha_{s}$ in the cohomology of $\mathcal{X}_{\mathbb{C}, s}$.

The class $\alpha_{s}$ only depends on the complex point $s$. Indeed, it can be obtained the following way. The class $\alpha$ pulls-back as a section $\alpha_{\mathbb{C}}$ of $\mathcal{H}_{\mathbb{C}}^{2 p}$ over $S_{\mathbb{C}}$. The class $\alpha_{s}$ is the value of $\alpha_{\mathbb{C}}$ at the point $s \in S(\mathbb{C})$.

The following rephrases the definition of a Hodge class.

Proposition 42. Assume that $\alpha_{\eta}$ is an absolute Hodge class. If $\alpha_{\eta}$ is absolute, then $\alpha_{s}$ is a Hodge class. Furthermore, in case $k=\mathbb{Q}, \alpha_{\eta}$ is absolute if and only if $\alpha_{s}$ is a Hodge class for all s induced by embeddings $\sigma: \mathbb{Q}(S) \rightarrow \mathbb{C}$.

We try to investigate the implications of the previous rephrasing.

Lemma 43. Assume the field $k$ is countable. Then the set of points $s \in S_{\mathbb{C}}(\mathbb{C})$ induced by embeddings of $k(S)$ in $\mathbb{C}$ over $k$ is dense in $S_{\mathbb{C}}(\mathbb{C})$ for the usual topology.

Proof. Say that a complex point of $S_{\mathbb{C}}$ is very general if it does not lie in any proper algebraic subset of $S_{\mathbb{C}}$ defined over $k$. Since $k$ is countable, the Baire theorem shows that the set of general points is dense in $S_{\mathbb{C}}(\mathbb{C})$ for the usual topology. 
Now consider a very general point $s$. There exists an embedding of $k(S)$ into $\mathbb{C}$ such that the associated complex point of $S_{\mathbb{C}}$ is $s$. Indeed, $s$ being very general exactly means that the image of the morphism

$$
\operatorname{Spec}(\mathbb{C}) \stackrel{s}{\longrightarrow} S_{\mathbb{C}} \longrightarrow S
$$

is $\eta$, the generic point of $S$, hence a morphism $\operatorname{Spec}(\mathbb{C}) \rightarrow \eta$ giving rise to $s$. This concludes the proof of the lemma.

We say that a complex point of $S_{\mathbb{C}}$ is very general if it lies in the aforementioned subset.

Theorem 44. Let $S$ be a smooth irreducible quasi-projective variety over a subfield $k$ of $\mathbb{C}$ with generic point $\eta$, and let $\pi: \mathcal{X} \rightarrow S$ be a smooth projective morphism. Let $p$ be an integer, and let $\alpha$ be a section of $\mathcal{H}^{2 p}$ over $S$.

(1) Assume the class $\alpha_{\eta} \in H^{2 p}\left(\mathcal{X}_{\eta} / k(S)\right)$ is absolute Hodge. Then $\alpha$ is flat for the Gauss-Manin connection and $\alpha_{\mathbb{C}}$ is a Hodge class at every complex point of $S_{\mathbb{C}}$.

(2) Assume that $k=\mathbb{Q}$. Then the class $\alpha_{\eta} \in H^{2 p}\left(\mathcal{X}_{\eta} / \mathbb{Q}(S)\right)$ is absolute Hodge if and only if $\alpha$ is flat for the Gauss-Manin connection and for any connected component $S^{\prime}$ of $S_{\mathbb{C}}$, there exists a complex point s of $S^{\prime}$ such that $\alpha_{s}$ is a Hodge class.

Proof. All the objects we are considering are defined over a subfield of $k$ that is finitely generated over $\mathbb{Q}$, so we can assume that $k$ is finitely generated over $\mathbb{Q}$, hence countable. Let $\alpha_{\mathbb{C}}$ be the section of $\mathcal{H}_{\mathbb{C}}^{2 p}$ over $S_{\mathbb{C}}$ obtained by pulling-back $\alpha$. The value of the class $\alpha_{\mathbb{C}}$ at any general point is a Hodge class. Locally on $S_{\mathbb{C}}$, the bundle $\mathcal{H}_{\mathbb{C}}^{2 p}$ with the Gauss-Manin connection is biholomorphic to the flat bundle $S \times \mathbb{C}^{n}, n$ being the rank of $\mathcal{H}_{\mathbb{C}}^{2 p}$, and we can assume such a trivialization respects the rational subspaces.

Under such trivializations, the section $\alpha_{\mathbb{C}}$ is given locally on $S_{\mathbb{C}}$ by $n$ holomorphic functions which take rational values on a dense subset. It follows that $\alpha_{\mathbb{C}}$ is locally constant, that is, that $\alpha_{\mathbb{C}}$, hence $\alpha$, is flat for the Gauss-Manin connection. Since $\alpha$ is absolute Hodge, $\alpha_{\mathbb{C}}$ is a Hodge class at any very general point of $S_{\mathbb{C}}$. Since those are dense in $S_{\mathbb{C}}(\mathbb{C})$, Proposition 34 shows that $\alpha_{\mathbb{C}}$ is a Hodge class at every complex point of $S_{\mathbb{C}}$. This proves the first part of the theorem.

For the second part, assuming $\alpha$ is flat for the Gauss-Manin connection and $\alpha_{s}$ is Hodge for points $s$ in ,all the connected components of $S_{\mathbb{C}}$, Proposition 34 shows that $\alpha_{s}$ is a Hodge class at all the complex points $s$ of $S_{\mathbb{C}}$. In particular, this true for the general points of $S_{\mathbb{C}}$, which proves that $\alpha_{\eta}$ is an absolute Hodge class by Proposition 42 .

As a corollary, we get the following important result.

Theorem 45. Let $k$ be an algebraically closed subfield of $\mathbb{C}$, and let $X$ be a smooth projective variety over $k$. Let $\alpha$ be an absolute Hodge class of degree $2 p$ in $X_{\mathbb{C}}$. Then $\alpha$ is defined over $k$, that is, $\alpha$ is the pull-back of an absolute Hodge class in $X$.

Proof. The cohomology class $\alpha$ belongs to $H^{2 p}\left(X_{\mathbb{C}} / \mathbb{C}\right)=H^{2 p}(X / k) \otimes \mathbb{C}$. We need to show that it lies in $H^{2 p}(X / k) \subset H^{2 p}\left(X_{\mathbb{C}} / \mathbb{C}\right)$, that is, that it is defined over $k$.

The class $\alpha$ is defined over a field $K$ finitely generated over $k$. Since $K$ is generated by a finite number of elements over $k$, we can find a smooth irreducible quasi-projective variety $S$ defined over $k$ such that $K$ is isomorphic to $k(S)$. Let $\mathcal{X}=X \times S$, and let $\pi$ be the projection of $\mathcal{X}$ onto $S$. Saying that $\alpha$ is defined over $k(S)$ means that $\alpha$ is a class defined at the generic fiber of $\pi$. Up to replacing $S$ by a Zariski-open subset, we can assume that $\alpha$ extends to a section $\widetilde{\alpha}$ of the relative de Rham cohomology group $\mathcal{H}^{2 p}$ of $\mathcal{X}$ over $S$. Since $\alpha$ is an absolute Hodge class, Theorem 44 shows that $\widetilde{\alpha}$ is flat with respect to the Gauss-Manin connection on $\mathcal{H}^{2 p}$. 
Since $\mathcal{X}=X \times S$, relative de Rham cohomology is trivial, that is, the flat bundle $\mathcal{H}^{2 p}$ is isomorphic to $H^{2 p}(X / k) \otimes \mathcal{O}_{S}$ with the canonical connection. Since $\widetilde{\alpha}$ is a flat section over $S$ which is irreducible over the algebraically closed field $k$, it corresponds to the constant section with value some $\alpha_{0}$ in $H^{2 p}(X / k)$. Then $\alpha$ is the image of $\alpha_{0}$ in $H^{2 p}\left(X_{\mathbb{C}} / \mathbb{C}\right)=H^{2 p}(X / k) \otimes \mathbb{C}$, which concludes the proof.

Remark 46. In case $\alpha$ is the cohomology class of an algebraic cycle, the preceding result is a consequence of the existence of Hilbert schemes. If $Z$ is an algebraic cycle in $X_{\mathbb{C}}, Z$ is algebraically equivalent to an algebraic cycle defined over $k$. Indeed, $Z$ corresponds to a point in some product of Hilbert schemes parameterizing subschemes of $X$. Those Hilbert schemes are defined over $k$, so their points with value in $k$ are dense. This shows the result. Of course, classes of algebraic cycles are absolute Hodge, so this is a special case of the previous result.

3.5. The field of definition of the locus of Hodge classes. In this paragraph, we present some of the results of Voisin in 31. While those could be proved using Principle B and the global invariant cycle theorem along a line of arguments we used earlier, we focus on deducing the theorems as consequences of statements from the previous paragraph. The reader can consult [33] for the former approach.

Let $S$ be a smooth complex quasi-projective variety, and let $\pi: \mathcal{X} \rightarrow S$ be a smooth projective morphism. Let $p$ be an integer, and let $\mathcal{H}^{2 p}=\mathbb{R}^{2 p} \pi_{*} \Omega_{\mathcal{X} / S}$ together with the Hodge filtration $F^{k} \mathcal{H}^{2 p}=\mathbb{R}^{2 p} \pi_{*} \Omega_{\mathcal{X} / S}^{\bullet}$. Assume $\pi$ is defined over $\mathbb{Q}$. Then $\mathcal{H}^{2 p}$ is defined over $\mathbb{Q}$, as well as the Hodge filtration. Inside $\mathcal{H}^{2 p}$, we have the locus of Hodge classes as before. It is an algebraic subset of $\mathcal{H}^{2 p}$.

Note that any smooth projective complex variety is isomorphic to the fiber of such a morphism $\pi$ over a complex point. Indeed, if $X$ is a smooth projective complex variety, it is defined over a field finitely generated over $\mathbb{Q}$. Noticing that such a field is the function field of a smooth quasiprojective variety $S$ defined over $\mathbb{Q}$ allows us to find $\mathcal{X} \rightarrow S$ as before. Note that $S$ might not be geometrically irreducible.

Theorem 47. Let $s$ be a complex point of $S$, and let $\alpha$ be a Hodge class in $H^{2 p}\left(\mathcal{X}_{s} / \mathbb{C}\right)$. Then $\alpha$ is an absolute Hodge class if and only if the connected component $Z_{\alpha}$ of the locus of Hodge classes passing through $\alpha$ is defined over $\overline{\mathbb{Q}}$ and the conjugates of $Z_{\alpha}$ by $\operatorname{Gal}(\overline{\mathbb{Q}} / \mathbb{Q})$ are contained in the locus of Hodge classes.

Proof. Let $Z^{\prime}$ be the smallest algebraic subset defined over $\mathbb{Q}$ containing $Z_{\alpha}$. It is the $\mathbb{Q}$-Zariski closure of $Z_{\alpha}$. We want to show that $Z^{\prime}$ is contained in the locus of Hodge classes if and only if $\alpha$ is absolute Hodge.

Pulling back to the image of $Z^{\prime}$ and spreading the base scheme $S$ if necessary, we can reduce to the situation where $Z^{\prime}$ dominates $S$, and there exists a smooth projective morphism

$$
\pi_{\mathbb{Q}}: \mathcal{X}_{\mathbb{Q}} \rightarrow S_{\mathbb{Q}}
$$

defined over $\mathbb{Q}$, such that $\pi$ is the pull-back of $\pi_{\mathbb{Q}}$ to $\mathbb{C}$, a class $\alpha_{\mathbb{Q}}$ in $H^{2 p}\left(\mathcal{X}_{\mathbb{Q}} / S\right)$ ), and a embedding of $\mathbb{Q}\left(S_{\mathbb{Q}}\right)$ into $\mathbb{C}$ corresponding to the complex point $s \in S(\mathbb{C})$ such that $\mathcal{X}_{s}$ and $\alpha$ are the pullback of $\mathcal{X}_{\mathbb{Q}, \eta}$ and $\alpha_{\eta}$ respectively, where $\eta$ is the generic point of $S$.

In this situation, by the definition of absolute Hodge classes, $\alpha$ is an absolute Hodge class if and only if $\alpha_{\eta}$ is. Also, since $Z^{\prime}$ dominates $S, Z^{\prime}$ is contained in the locus of Hodge classes if and only if $\alpha$ extends as a flat section of $\mathcal{H}^{2 p}$ over $S$ which is a Hodge class at every complex point. Such 
a section is automatically defined over $\mathbb{Q}$ since the Gauss-Manin connection is. Statement (2) of Theorem 44 allows us to conclude the proof.

Remark 48. It is to be noted that the proof uses in an essential way the theorem of Cattani-DeligneKaplan on the algebraicity of Hodge loci.

Recall that Conjecture 25 predicts that Hodge classes are absolute. As an immediate consequence, we get the following reformulation.

Corollary 49. Conjecture 25 is equivalent to the following.

Let $S$ be a smooth complex quasi-projective variety, and let $\pi: \mathcal{X} \rightarrow S$ be a smooth projective morphism. Assume $\pi$ is defined over $\mathbb{Q}$. Then the locus of Hodge classes for $\pi$ is a countable union of algebraic subsets of the Hodge bundles defined over $\mathbb{Q}$.

It is possible to prove the preceding corollary without resorting to the Cattani-Deligne-Kaplan theorem, see Proposition 40.

In the light of this result, the study of whether Hodge classes are absolute can be seen as a study of the field of definition of the locus of Hodge classes. An intermediate property is to a ask for the component of the locus of Hodge classes passing through a class $\alpha$ to be defined over $\bar{Q}$. In [31, Voisin shows how one can use arguments from the theory of variations of Hodge structures to give infinitesimal criteria for this to happen.

This is closely related to the rigidity result of Theorem 45. Indeed, using the fact that the Gauss-Manin connection is defined over $\mathbb{Q}$, it is easy to show that the component of the locus of Hodge classes passing through a class $\alpha$ in the cohomology of a complex variety defined over $\overline{\mathbb{Q}}$ is defined over $\bar{Q}$ if and only if $\alpha$ is defined over $\overline{\mathbb{Q}}$ as a class in algebraic de Rham cohomology.

Let us conclude this section by showing how the study of fields of definition for Hodge loci is related to the Hodge conjecture. The following is due to Voisin in 31 .

Theorem 50. Let $S$ be a smooth complex quasi-projective variety, and let $\pi: \mathcal{X} \rightarrow S$ be a smooth projective morphism. Assume $\pi$ is defined over $\mathbb{Q}$. Let $s$ be a complex point of $S$ and let $\alpha$ be a Hodge class in $H^{2 p}\left(\mathcal{X}_{s}, \mathbb{Q}(p)\right.$. If the image in $S$ of the component of the locus of Hodge classes passing through $\alpha$ is defined over $\overline{\mathbb{Q}}$, then the Hodge conjecture for $\alpha$ can be reduced to the Hodge conjecture for varieties defined over number fields.

Proof. This is a consequence of the global invariant cycle theorem. Indeed, with the notation of Theorem 33, one can choose the compactification $\overline{\mathcal{X}}$ to be defined over $\overline{\mathbb{Q}}$. The desired result follows easily.

\section{The Kuga-Satake construction}

In this section, we give our first nontrivial example of absolute Hodge classes. It is due to Deligne in 14 .

Let $S$ be a complex projective K3 surface. We want construct an abelian variety $A$ and an embedding of Hodge structures

$$
H^{2}(S, \mathbb{Q}) \hookrightarrow H^{1}(A, \mathbb{Q}) \otimes H^{1}(A)
$$

which is absolute Hodge. This is the Kuga-Satake correspondence, see 24], 14.

We will take a representation-theoretic approach to this problem. This paragraph merely outlines the construction of the Kuga-Satake correspondence, leaving aside part of the proofs. We refer to 
the survey [19] for more details. Properties of Spin groups and their representations can be found in [18], Chapter 20 or [6], Paragraph 9.

4.1. Recollection on Spin groups. We follow Deligne's approach in [14. Let us start with some linear algebra. Let $V$ be a finite-dimensional vector space over a field $k$ of characteristic zero with a non-degenerate quadratic form $Q$. Recall that the Clifford algebra $C(V)$ over $V$ is the algebra defined as the quotient of the tensor algebra $\bigoplus_{i \leq 0} V^{\otimes i}$ by the relation $v \otimes v=Q(v), v \in V$. Even though the natural grading of the tensor algebra does not descend to the Clifford algebra, there is a well-defined sub-algebra $C^{+}(V)$ of $C(V)$ which is the image of $\bigoplus_{i \leq 0} V^{\otimes 2 i}$ in $C(V)$. The algebra $C^{+}(V)$ is the even Clifford algebra over $V$.

The Clifford algebra is endowed with an anti-automorphism $x \mapsto x^{*}$ such that $\left(v_{1} \ldots v_{i}\right)^{*}=$ $v_{i} \ldots v_{1}$ if $v_{1}, \ldots, v_{i} \in V$. The Clifford group of $V$ is the algebraic group defined by

$$
C \operatorname{Spin}(V)=\left\{x \in C^{+}(V)^{*}, x \cdot V \cdot x^{-1} \subset V\right\} .
$$

It can be proved that $C \operatorname{Spin}(V)$ a connected algebraic group. By definition, it acts on $V$. Let $x \in C \operatorname{Spin}(V), v \in V$. We have $Q\left(x v x^{-1}\right)=x v x^{-1} x v x^{-1}=x Q(v) x^{-1}=Q(v)$, which shows that $C \operatorname{Spin}(V)$ acts on $V$ through the orthogonal group $O(V)$, hence a map from $C \operatorname{Spin}(V)$ to $O(V)$. Since $C \operatorname{Spin}(V)$ is connected, this map factors through $\tau: C \operatorname{Spin}(V) \rightarrow S O(V)$. We have an exact sequence

$$
1 \longrightarrow \mathbb{G}_{m} \stackrel{w}{\longrightarrow} C \operatorname{Spin}(V) \longrightarrow S O(V) \longrightarrow 1
$$

The spinor norm is the morphism of algebraic groups

$$
N: C \operatorname{Spin}(V) \rightarrow \mathbb{G}_{m}, x \mapsto x x^{*} .
$$

It is well-defined. Let $t$ be the inverse of $N$. The composite map

$$
t \circ w: \mathbb{G}_{m} \rightarrow \mathbb{G}_{m}
$$

is the map $x \mapsto x^{-2}$. The Spin group $\operatorname{Spin}(V)$ is the algebraic group defined as the kernel of $N$. The Clifford group is generated by homotheties and elements of the Spin group.

The Spin group is connected and simply connected. The exact sequence

$$
1 \rightarrow \pm 1 \rightarrow \operatorname{Spin}(V) \rightarrow S O(V) \rightarrow 1
$$

realizes the Spin group as the universal covering of $S O(V)$.

4.2. Spin representations. The Clifford group has two different representations on $C^{+}(V)$. The first one is the adjoint representation $C^{+}(V)_{a d}$. The adjoint action of $C \operatorname{Spin}(V)$ is defined as

$$
x \cdot a d v=x v x^{-1}
$$

where $x \in C \operatorname{Spin}(V), v \in C^{+}(V)$. It factors through $S O(V)$ and is isomorphic to $\bigoplus_{i} \bigwedge^{2 i} V$ as a representation of $C \operatorname{Spin}(V)$.

The group $C \operatorname{Spin}(V)$ acts on $C^{+}(V)$ by multiplication on the left, hence a representation $C^{+}(V)_{s}$, with

$$
x \cdot s v=x v,
$$

where $x \in C \operatorname{Spin}(V), v \in C^{+}(V)$. It is compatible with the structure of right $C^{+}(V)$-module on $C^{+}(V)$, and we have

$$
\operatorname{End}_{C^{+}(V)}\left(C^{+}(V)_{s}\right)=C^{+}(V)_{a d} .
$$

Assume $k$ is algebraically closed. We can describe those representations explicitly. In case the dimension of $V$ is odd, let $W$ be a simple $C^{+}(V)$-module. The Clifford group $C \operatorname{Spin}(V)$ acts on 
$W$. This is the spin representation of $C \operatorname{Spin}(V)$. Then $C^{+}(V)_{s}$ is isomorphic to a sum of copy of $W$, and $C^{+}(V)_{a d}$ is isomorphic to $\operatorname{End}_{k}(W)$ as representations of $C \operatorname{Spin}(V)$.

In case the dimension of $V$ is even, let $W_{1}$ and $W_{2}$ be nonisomorphic simple $C^{+}(V)$-modules. Those are the half-spin representations of $C \operatorname{Spin}(V)$. Their sum $W$ is called the spin representation. Then $C^{+}(V)_{s}$ is isomorphic to a sum of copy of $W$, and $C^{+}(V)_{a d}$ is isomorphic to $\operatorname{End}_{k}\left(W_{1}\right) \times$ $\operatorname{End}_{k}\left(W_{2}\right)$ as representations of $C \operatorname{Spin}(V)$.

4.3. Hodge structures and the Deligne torus. Recall the definition of Hodge structures à la Deligne, see [13. Let $S$ be the Deligne torus, that is, the real algebraic group of invertible elements of $\mathbb{C}$. It can be defined as the Weil restriction of $\mathbb{G}_{m}$ from $\mathbb{C}$ to $\mathbb{R}$. We have an exact sequence of real algebraic groups

$$
1 \longrightarrow \mathbb{G}_{m} \stackrel{w}{\longrightarrow} S \stackrel{t}{\longrightarrow} \mathbb{G}_{m} \longrightarrow 1
$$

where $w$ is the inclusion of $\mathbb{R}^{*}$ into $\mathbb{C}^{*}$ and $t$ maps a complex number $z$ to $|z|^{-2}$. The composite map

is the map $x \mapsto x^{-2}$.

$$
t \circ w: \mathbb{G}_{m} \rightarrow \mathbb{G}_{m}
$$

Let $V_{\mathbb{Z}}$ be a free $\mathbb{Z}$-module of finite rank, and let $V=V_{\mathbb{Q}}$. The datum of a Hodge structure of weight $k$ on $V$ (or $V_{\mathbb{Z}}$ ) is the same as the datum of a representation $\rho: S \rightarrow G L\left(V_{\mathbb{R}}\right)$ such that $\rho w(x)=x^{k} I d_{V_{\mathbb{R}}}$ for all $x \in \mathbb{R}^{*}$. Given a Hodge structure of weight $n, z \in \mathbb{C}^{*}$ acts on $V_{\mathbb{R}}$ by $z \cdot v=z^{p} \bar{z}^{q} v$ if $v \in V^{p, q}$.

4.4. From weight two to weight one. Now assume $V$ is polarized of weight zero with Hodge numbers $V^{-1,1}=V^{1,-1}=1, V^{0,0}=0$. We say that $V$ (or $V_{\mathbb{Z}}$ ) is of $\mathrm{K} 3$ type. We get a quadratic form $Q$ on $V_{\mathbb{R}}$, and the representation of $S$ on $V_{\mathbb{R}}$ factors through the special orthogonal group of $V$ as $h: S \rightarrow S O\left(V_{\mathbb{R}}\right)$.

Lemma 51. There exists a unique lifting of $h$ to a morphism $\widetilde{h}: S \rightarrow C \operatorname{Spin}\left(V_{\mathbb{R}}\right)$ such that the following diagram commutes.

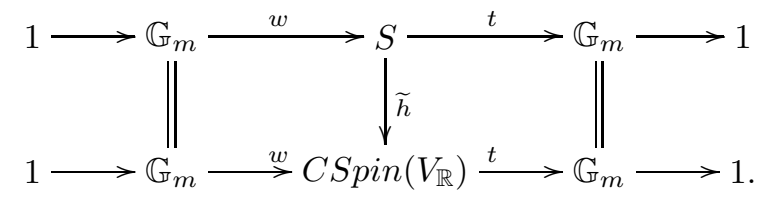

Proof. It is easy to prove that such a lifting is unique if it exists. The restriction of $Q$ to $P=$ $V_{\mathbb{R}} \cap\left(V^{-1,1} \oplus V^{1,-1}\right)$ is positive definite. Furthermore, $P$ has a canonical orientation. Let $e_{1}, e_{2}$ be a direct orthonormal basis of $P$. We have $e_{1} e_{2}=-e_{2} e_{1}$ and $e_{1}^{2}=e_{2}^{2}=1$. As a consequence, $\left(e_{2} e_{1}\right)^{2}=-1$. An easy computation shows that the morphism $a+i b \mapsto a+b e_{2} e_{1}$ defines a suitable lifting of $h$.

Using the preceding lemma, consider such a lifting $\widetilde{h}: S \rightarrow C \operatorname{Spin}(V)$ of $h$. Any representation of $C \operatorname{Spin}\left(V_{\mathbb{R}}\right)$ thus gives rise to a Hodge structure. Let us first consider the adjoint representation. We know that $C^{+}(V)_{a d}$ is isomorphic to $\bigoplus_{i} \wedge^{2 i} V$, where $C \operatorname{Spin}(V)$ acts on $V$ through $S O(V)$. It follows that $\widetilde{h}$ endows $C^{+}(V)_{a d}$ with a weight zero Hodge structure. Since $V^{-1,1}=1$, the type of the Hodge structure $C^{+}(V)_{a d}$ is $\{(-1,1),(0,0),(1,-1)\}$.

Now assume the dimension of $V$ is odd, and consider the spin representation $W$. It is a weight one representation. Indeed, the lemma above shows that $C^{+}(V)_{s}$ is of weight one, and it is isomorphic 
to a sum of copies of $W$. Since $C^{+}(V)_{a d}$ is isomorphic to $\operatorname{End}_{k}(W)$ as representations of $C \operatorname{Spin}(V)$, the type of $W$ is $\{(1,0),(0,1)\}$.

It follows that $\widetilde{h}$ endows $C^{+}(V)_{s}$ with an effective Hodge structure of weight one. It is possible to show that this Hodge structure is polarizable, see [14. The underlying vector space has $C^{+}\left(V_{\mathbb{Z}}\right)$ as a natural lattice. This construction thus defines an abelian variety. Similar computations show that the same result holds if the dimension of $V$ is even.

Definition 52. The abelian variety defined by the Hodge structure on $C^{+}(V)_{s}$ with its natural lattice $C^{+}\left(V_{\mathbb{Z}}\right)$ is called the Kuga-Satake variety associated to $V_{\mathbb{Z}}$. We denote it by $K S\left(V_{\mathbb{Z}}\right)$.

Theorem 53. Let $V_{\mathbb{Z}}$ be a polarized Hodge structure of K3 type. There exists a natural injective morphism of Hodge structures

$$
V_{\mathbb{Q}}(-1) \hookrightarrow H^{1}\left(K S\left(V_{\mathbb{Z}}\right), \mathbb{Q}\right) \otimes H^{1}\left(K S\left(V_{\mathbb{Z}}\right), \mathbb{Q}\right) .
$$

This morphism is called the Kuga-Satake correspondence.

Proof. Let $V=V_{\mathbb{Q}}$. Fix an element $v_{0} \in V$ and consider the vector space $M=C^{+}(V)$. It is endowed with a left action of $V$ by the formula

$$
v \cdot x=v x v_{0}
$$

for $v \in V, x \in C^{+}(V)$. This action induces an embeddings

$$
V \hookrightarrow \operatorname{End}_{\mathbb{Q}}\left(C^{+}(V)_{s}\right)
$$

which is equivariant with respect to the action of $C \operatorname{Spin}(V)$.

Now we can consider $\operatorname{End}_{\mathbb{Q}}\left(C^{+}(V)_{s}\right)(-1)$ as a subspace of $C^{+}(V) \otimes C^{+}(V)=H^{1}\left(K S\left(V_{\mathbb{Z}}\right), \mathbb{Q}\right) \otimes$ $H^{1}\left(K S\left(V_{\mathbb{Z}}\right), \mathbb{Q}\right)$ via a polarization of $K S\left(V_{\mathbb{Z}}\right)$, and $V$ as a subspace of $C^{+}(V)$. This gives an injection

$$
V(-1) \hookrightarrow H^{1}\left(K S\left(V_{\mathbb{Z}}\right), \mathbb{Q}\right) \otimes H^{1}\left(K S\left(V_{\mathbb{Z}}\right), Q\right)
$$

as desired. The equivariance property stated above shows that this is a morphism of Hodge structures.

Remark 54. Let $V$ be a Hodge structure of K3 type. In order to construct the Kuga-Satake correspondence associated to $V$, we can relax a bit the assumption that $V$ is polarized. Indeed, it is enough to assume that $V$ is endowed with a quadratic form that is positive definite on $\left(V^{-1,1} \oplus\right.$ $\left.V^{1,-1}\right) \bigcap V_{\mathbb{R}}$ and such that $V^{1,-1}$ and $V^{-1,1}$ are totally isotropic subspaces of $V$.

4.5. The Kuga-Satake correspondence is absolute. Let $X$ be a polarized complex $K 3$ surface. Denote by $K S(X)$ the Kuga-Satake variety associated to $H^{2}(X, \mathbb{Z}(1))$ endowed with the intersection pairing. Even though this pairing only gives a polarization on the primitive part of cohomology, the construction is possible by the preceding remark. Theorem 53 gives us a correspondence between the cohomology groups of $X$ and its Kuga-Satake variety. This is the Kuga-Satake correspondence for $X$. We can now state and prove the main theorem of this section. It is proved by Deligne in [14.

Theorem 55. Let $X$ be a polarized complex K3 surface. The Kuga-Satake correspondence

$$
H^{2}(X, \mathbb{Q}(1)) \hookrightarrow H^{1}(K S(X), \mathbb{Q}) \otimes H^{1}(K S(X), \mathbb{Q})
$$

is absolute Hodge. 
Proof. Any polarized complex $K 3$ surface deforms to a polarized Kummer surface in a polarized family. Now the Kuga-Satake construction works in families. As a consequence, by Principle B, see Theorem [36, it is enough to prove that the Kuga-Satake correspondence is absolute Hodge for a variety $X$ which is the Kummer variety associated to an abelian surface $A$. In this case, we can even prove the Kuga-Satake correspondence is algebraic. Let us outline the proof of this result, which has first been proved by Morrison in [26. We follow a slightly different path.

First, remark that the canonical correspondence between $A$ and $X$ identifies the transcendental part of the Hodge structure $H^{2}(X, \mathbb{Z}(1))$ with the transcendental part of $H^{2}(A, \mathbb{Z}(1))$. Note that the latter Hodge structure is of K3 type. Since this isomorphism is induced by an algebraic correspondence between $X$ and $A$, standard reductions show that it is enough to show that the Kuga-Satake correspondence between $A$ and the Kuga-Satake abelian variety associated to $H^{2}(A, \mathbb{Z}(1))$ is algebraic. Let us write $U=H^{1}(A, \mathbb{Q})$ and $V=H^{2}(A, \mathbb{Q})$, considered as vector spaces.

We have $V=\bigwedge^{2} U$. The vector space $U$ is of dimension 2, and the weight 1 Hodge structure on $U$ induces a canonical isomorphism $\bigwedge^{2} V=\bigwedge^{4} U \simeq \mathbb{Q}$. The intersection pairing $Q$ on $V$ satisfies

$$
\forall x, y \in V, Q(x, y)=x \wedge y .
$$

Let $g \in S L(U)$. The determinant $g$ being $1, g$ acts trivially on $\bigwedge^{2} V=\bigwedge^{4} U$. As a consequence, $g \wedge g$ preserves the intersection form on $V$. This gives a morphism $S L(U) \rightarrow S O(V)$. The kernel of this morphism is $\pm I d_{U}$, and it is surjective by dimension counting. Since $S L(U)$ is a connected algebraic group, this gives a canonical isomorphism $S L(U) \simeq \operatorname{Spin}(V)$.

The group $S L(U)$ acts on $U$ by the standard action and on its dual $U^{*}$ by $g \mapsto{ }^{t} g^{-1}$. Those representations are irreducible, and they are not isomorphic since no nontrivial bilinear form on $U$ is preserved by $S L(U)$. By standard representation theory, those are the two half-spin representations of $S L(U) \simeq \operatorname{Spin}(V)$. As a consequence, the Clifford algebra of $V$ is canonically isomorphic to $\operatorname{End}(U) \times \operatorname{End}\left(U^{*}\right)$, and we have a canonical identification

$$
C \operatorname{Spin}(V)=\left\{\left(\lambda g, \lambda^{t} g^{-1}\right), g \in S L(U), \lambda \in \mathbb{G}_{m}\right\} .
$$

An element $\left(\lambda g, \lambda^{t} g^{-1}\right)$ of the Clifford group acts on the half-spin representations $U$ and $U^{*}$ by the first and second component respectively.

The preceding identifications allow us to conclude the proof. Let $h^{\prime}: S \rightarrow G L(U)$ be the morphism that defines the weight one Hodge structure on $U$, and let $h: S \rightarrow S O(V)$ endow $V$ with its Hodge structure of K3 type. Note that if $s \in \mathbb{C}^{*}$, the determinant of $h^{\prime}(s)$ is $|s|^{4}$ since $U$ is of dimension 4 and weight 1 . Since $V=\bigwedge^{2} U(1)$ as Hodge structures, we get that $h$ is the morphism

$$
h: s \mapsto|s|^{-2} h^{\prime}(s) \wedge h^{\prime}(s) \text {. }
$$

It follows that the morphism

$$
\widetilde{h}: S \rightarrow C \operatorname{Spin}(V), s \mapsto\left(h^{\prime}(s),|s|^{2 t} h^{\prime}(s)^{-1}\right)=\left(|s||s|^{-1} h^{\prime}(s) s,|s|^{t}\left(|s|^{-1} h^{\prime}(s)\right)^{-1}\right)
$$

is a lifting of $h$ to $C \operatorname{Spin}(V)$.

Following the previous identifications shows that the Hodge structure induced by $\widetilde{h}$ on $U$ and $U^{*}$ are the ones induced by the identifications $U=H^{1}(A, \mathbb{Q})$ and $U^{*}=H^{1}(\hat{A}, \mathbb{Q})$, where $\hat{A}$ is the dual abelian variety. Since the representation $C^{+}(V)_{s}$ is a sum of 4 copies of $U \oplus U^{*}$, this gives an isogeny between $K S(A)$ and $(A \times \hat{A})^{4}$ and shows that the Kuga-Satake correspondence is algebraic, using the identity correspondence between $A$ and itself and the correspondence between $A$ and its dual induced by the polarization. This concludes the proof. 
Remark 56. Since the cohomology of a Kummer variety is a direct factor of the cohomology of an abelian variety, it is an immediate consequence of Deligne's theorem on absolute cycles on abelian varieties that the Kuga-Satake correspondence for Kummer surfaces is absolute Hodge. However, our proof is more direct and also gives the algebraicity of the correspondence in the Kummer case. Few algebraicity results are known for the Kuga-Satake correspondence, but see 27] for the case of K3 surfaces which are a double cover of $\mathbb{P}^{2}$ ramified over 6 lines. See also [19] and 30] for further discussion of this problem.

Remark 57. In Definition 10, we extended the notion of absolute Hodge classes to the setting of étale cohomology. While we did not use this notion, most results we stated, for instance Principle $\mathrm{B}$, can be generalized in this setting with little additional work. This makes it possible to show that the Kuga-Satake correspondence is absolute Hodge in the sense of Definition [10. In the paper [14, Deligne uses this to deduce the Weil conjectures for K3 surfaces from the Weil conjectures for abelian varieties.

\section{Deligne's theorem on Hodge Classes on abelian varieties}

Having introduced the notion of absolute Hodge classes, Deligne went on to prove the following remarkable theorem, which has already been mentioned several times in these notes.

Theorem 58 (Deligne). On an abelian variety, all Hodge classes are absolute.

The purpose of the remaining lectures is to explain the proof of Deligne's theorem. We follow Milne's account of the proof [16], with some simplifications due to André [2] and Voisin.

5.1. Overview. In the lectures of Griffiths and Kerr, we have already seen that rational Hodge structures whose endomorphism algebra contains a CM-field are very special. Since abelian varieties of CM-type also play a crucial role in the proof of Deligne's theorem, we shall begin by recalling two basic definitions.

Definition 59. A CM field is a number field $E$ that admits an involution $\iota \in \operatorname{Gal}(E / \mathbb{Q})$, such that for any embedding $s: E \hookrightarrow \mathbb{C}$, one has $\bar{s}=s \circ \iota$.

The fixed field of the involution is a totally real field $F$; concretely, this means that $F=\mathbb{Q}(\alpha)$, where $\alpha$ and all of its conjugates are real numbers. The field $E$ is then of the form $F[x] /\left(x^{2}-f\right)$, for some element $f \in F$ that is mapped to a negative number under all embeddings of $F$ into $\mathbb{R}$.

Definition 60. An abelian variety $A$ is said to be of CM-type if a CM-field $E$ is contained in $\operatorname{End}(A) \otimes \mathbb{Q}$, and if $H^{1}(A, \mathbb{Q})$ is one-dimensional as an E-vector space. In that case, we clearly have $2 \operatorname{dim} A=\operatorname{dim}_{\mathbb{Q}} H^{1}(A, \mathbb{Q})=[E: \mathbb{Q}]$.

We will carry out a more careful analysis of abelian varieties and Hodge structures of CMtype below. To motivate what follows, let us however briefly look at a criterion for a simple abelian variety $A$ to be of CM-type that involves the (special) Mumford-Tate group $\operatorname{MT}(A)=\operatorname{MT}\left(H^{1}(A)\right)$.

Recall that the Hodge structure on $H^{1}(A, \mathbb{Q})$ can be described by a morphism of $\mathbb{R}$-algebraic groups $h: U(1) \rightarrow \operatorname{GL}\left(H^{1}(A, \mathbb{R})\right)$; the weight being fixed, $h(z)$ acts as multiplication by $z^{p-q}$ on the space $H^{p, q}(A)$. Recalling Paragraph 4.3, the group $U(1)$ is the kernel of the weight $w: S \rightarrow \mathbb{G}_{m}$. Representations of $\operatorname{Ker}(w)$ correspond to Hodge structures of fixed weight.

We can define $\operatorname{MT}(A)$ as the smallest $\mathbb{Q}$-algebraic subgroup of $\operatorname{GL}\left(H^{1}(A, \mathbb{Q})\right)$ whose set of real points contains the image of $h$. Equivalently, it is the subgroup fixing every Hodge class in every tensor product

$$
T^{p, q}(A)=H^{1}(A)^{\otimes p} \otimes H_{1}(A)^{\otimes q} .
$$


We have the following criterion.

Proposition 61. A simple abelian variety is of CM-type if and only if its Mumford-Tate group $\operatorname{MT}(A)$ is an abelian group.

Here is a quick outline of the proof of the interesting implication.

Proof. Let $H=H^{1}(A, \mathbb{Q})$. The abelian variety $A$ is simple, which implies that $E=\operatorname{End}(A) \otimes \mathbb{Q}$ is a division algebra. It is also the space of Hodge classes in $\operatorname{End}_{\mathbb{Q}}(H)$, and therefore consists exactly of those endomorphisms that commute with $\operatorname{MT}(A)$. Because the Mumford-Tate group is abelian, its action splits $H^{1}(A, \mathbb{C})$ into a direct sum of character spaces

$$
H \otimes_{\mathbb{Q}} \mathbb{C}=\bigoplus_{\chi} H_{\chi}
$$

where $m \cdot h=\chi(m) h$ for $h \in H_{\chi}$ and $m \in \mathrm{MT}(A)$. Now any endomorphism of $H_{\chi}$ obviously commutes with $\operatorname{MT}(A)$, and is therefore contained in $E \otimes_{\mathbb{Q}} \mathbb{C}$. By counting dimensions, we find that

$$
\operatorname{dim}_{\mathbb{Q}} E \geq \sum_{\chi}\left(\operatorname{dim}_{\mathbb{C}} H_{\chi}\right)^{2} \geq \sum_{\chi} \operatorname{dim}_{\mathbb{C}} H_{\chi}=\operatorname{dim}_{\mathbb{Q}} H .
$$

On the other hand, we have $\operatorname{dim}_{\mathbb{Q}} E \leq \operatorname{dim}_{\mathbb{Q}} H$; indeed, since $E$ is a division algebra, the map $E \rightarrow H, e \mapsto e \cdot h$, is injective for every nonzero $h \in H$. Therefore $[E: \mathbb{Q}]=\operatorname{dim}_{\mathbb{Q}} H=2 \operatorname{dim} A$; moreover, each character space $H_{\chi}$ is one-dimensional, and this implies that $E$ is commutative, hence a field. To construct the involution $\iota: E \rightarrow E$ that makes $E$ into a CM-field, choose a polarization $\psi: H \times H \rightarrow \mathbb{Q}$, and define $\iota$ by the condition that, for every $h, h^{\prime} \in H$,

$$
\psi\left(e \cdot h, h^{\prime}\right)=\psi\left(h, \iota(e) \cdot h^{\prime}\right) .
$$

The fact that $-i \psi$ is positive definite on the subspace $H^{1,0}(A)$ can then be used to show that $\iota$ is nontrivial, and that $\bar{s}=s \circ \iota$ for any embedding of $E$ into the complex numbers.

After this preliminary discussion of abelian varieties of CM-type, we return to Deligne's theorem on an arbitrary abelian variety $A$. The proof consists of the following three steps.

1. The first step is to reduce the problem to abelian varieties of CM-type. This is done by constructing an algebraic family of abelian varieties that links a given $A$ and a Hodge class in $H^{2 p}(A, \mathbb{Q})$ to an abelian variety of CM-type and a Hodge class on it, and then applying Principle B.

2. The second step is to show that every Hodge class on an abelian variety of CM-type can be expressed as a sum of pullbacks of so-called split Weil classes. The latter are Hodge classes on certain special abelian varieties, constructed by linear algebra from the CM-field $E$ and its embeddings into $\mathbb{C}$. This part of the proof is due to André [2].

3. The last step is to show that all split Weil classes are absolute. For a fixed CM-type, all abelian varieties of split Weil type are naturally parametrized by a certain hermitian symmetric domain; by Principle B, this allows to reduce the problem to showing that split Weil classes on abelian varieties of a very specific form for which the result is straightforward.

The original proof by Deligne uses Baily-Borel theory to show that certain families of abelian varieties are algebraic. Following a suggestion by Voisin, we have chosen to replace this by the following two results: the existence of a quasi-projective moduli space for polarized abelian varieties with level structure and the theorem of Cattani-Deligne-Kaplan in [10] concerning the algebraicity of Hodge loci. 
5.2. Hodge structures of CM-type. When $A$ is an abelian variety of CM-type, $H^{1}(A, \mathbb{Q})$ is an example of a Hodge structure of CM-type. We now undertake a more careful study of this class of Hodge structures. Let $V$ be a rational Hodge structure of weight $n$, with Hodge decomposition

$$
V \otimes_{\mathbb{Q}} \mathbb{C}=\bigoplus_{p+q=n} V^{p, q}
$$

Once we fix the weight $n$, there is a one-to-one correspondence between such decompositions and group homomorphisms $h: U(1) \rightarrow \mathrm{GL}\left(V \otimes_{\mathbb{Q}} \mathbb{R}\right)$. Namely, $h(z)$ acts as multiplication by $z^{p-q}=$ $z^{2 p-n}$ on the subspace $V^{p, q}$. We define the (special) Mumford-Tate group $\operatorname{MT}(V)$ as the smallest $\mathbb{Q}$-algebraic subgroup of $\mathrm{GL}(V)$ whose set of real points contains the image of $h$.

Definition 62. We say that $V$ is a Hodge structure of CM-type if the following two equivalent conditions are satisfied:

(a) The set of real points of $\mathrm{MT}(V)$ is a compact torus.

(b) $\operatorname{MT}(V)$ is abelian and $V$ is polarizable.

It is not hard to see that any Hodge structure of CM-type is a direct sum of irreducible Hodge structures of CM-type. Indeed, since $V$ is polarizable, it admits a finite decomposition $V=V_{1} \oplus$ $\cdots \oplus V_{r}$, with each $V_{i}$ irreducible. As subgroups of GL $(V)=\mathrm{GL}\left(V_{1}\right) \times \cdots \times \mathrm{GL}\left(V_{r}\right)$, we then have $\operatorname{MT}(V) \subseteq \operatorname{MT}\left(V_{1}\right) \times \cdots \times \operatorname{MT}\left(V_{r}\right)$, and since the projection to each factor is surjective, it follows that $\operatorname{MT}\left(V_{i}\right)$ is abelian. But this means that each $V_{i}$ is again of CM-type. It is therefore sufficient to concentrate on irreducible Hodge structures of CM-type. For those, there is a nice structure theorem that we shall now explain.

Let $V$ be an irreducible Hodge structure of weight $n$ that is of CM-type, and as above, denote by $\operatorname{MT}(V)$ its special Mumford-Tate group. Because $V$ is irreducible, its algebra of endomorphisms

$$
E=\operatorname{End}_{\mathbb{Q}-\mathrm{HS}}(V)
$$

must be a division algebra. In fact, since the endomorphisms of $V$ as a Hodge structure are exactly the Hodge classes in $\operatorname{End}_{\mathbb{Q}}(V)$, we see that $E$ consists of all rational endomorphisms of $V$ that commute with $\operatorname{MT}(V)$. If $T_{E}=E^{\times}$denotes the algebraic torus in $\operatorname{GL}(V)$ determined by $E$, then we get $\operatorname{MT}(V) \subseteq T_{E}$ because $\operatorname{MT}(V)$ is commutative by assumption.

Since $\mathrm{MT}(V)$ is commutative, it acts on $V \otimes_{\mathbb{Q}} \mathbb{C}$ by characters, and so we get a decomposition

$$
V \otimes_{\mathbb{Q}} \mathbb{C}=\bigoplus_{\chi} V_{\chi}
$$

where $m \in \operatorname{MT}(V)$ acts on $v \in V_{\chi}$ by the rule $m \cdot v=\chi(m) v$. Any endomorphism of $V_{\chi}$ therefore commutes with $\operatorname{MT}(V)$, and so $E \otimes_{\mathbb{Q}} \mathbb{C}$ contains the spaces $\operatorname{End}_{\mathbb{C}}\left(V_{\chi}\right)$. This leads to the inequality

$$
\operatorname{dim}_{\mathbb{Q}} E \geq \sum_{\chi}\left(\operatorname{dim}_{\mathbb{C}} V_{\chi}\right)^{2} \geq \sum_{\chi} \operatorname{dim}_{\mathbb{C}} V_{\chi}=\operatorname{dim}_{\mathbb{Q}} V
$$

On the other hand, we have $\operatorname{dim}_{\mathbb{Q}} V \leq \operatorname{dim}_{\mathbb{Q}} E$ because every nonzero element in $E$ is invertible. It follows that each $V_{\chi}$ is one-dimensional, that $E$ is commutative, and therefore that $E$ is a field of degree $[E: \mathbb{Q}]=\operatorname{dim}_{\mathbb{Q}} V$. In particular, $V$ is one-dimensional as an $E$-vector space.

The decomposition into character spaces can be made more canonical in the following way. Let $S=\operatorname{Hom}(E, \mathbb{C})$ denote the set of all complex embeddings of $E$; its cardinality is $[E: \mathbb{Q}]$. Then

$$
E \otimes \mathbb{Q} \mathbb{C} \stackrel{\sim}{\rightarrow} \bigoplus_{s \in S} \mathbb{C}, \quad e \otimes z \mapsto \sum_{s \in S} s(e) z,
$$


is an isomorphism of $E$-vector spaces; $E$ acts on each summand on the right through the corresponding embedding $s$. This decomposition induces an isomorphism

$$
V \otimes_{\mathbb{Q}} \mathbb{C} \stackrel{\sim}{\rightarrow} \bigoplus_{s \in S} V_{s}
$$

where $V_{s}=V \otimes_{E, s} \mathbb{C}$ is a one-dimensional complex vector space on which $E$ acts via $s$. The induced homomorphism $U(1) \rightarrow \operatorname{MT}(V) \rightarrow E^{\times} \rightarrow \operatorname{End}_{\mathbb{C}}\left(V_{s}\right)$ is a character of $U(1)$, hence of the form $z \mapsto z^{k}$ for some integer $k$. Solving $k=p-q$ and $n=p+q$, we find that $k=2 p-n$, which means that $V_{s}$ is of type $(p, n-p)$ in the Hodge decomposition of $V$. Now define a function $\varphi: S \rightarrow \mathbb{Z}$ by setting $\varphi(s)=p$; then any choice of isomorphism $V \simeq E$ puts a Hodge structure of weight $n$ on $E$, whose Hodge decomposition is given by

$$
E \otimes_{\mathbb{Q}} \mathbb{C} \simeq \bigoplus_{s \in S} \mathbb{C}^{\varphi(s), n-\varphi(s)} .
$$

From the fact that $\overline{e \otimes z}=e \otimes \bar{z}$, we deduce that

$$
\overline{\sum_{s \in S} z_{s}}=\sum_{s \in S} \overline{z_{\bar{s}}}
$$

Since complex conjugation has to interchange $\mathbb{C}^{p, q}$ and $\mathbb{C}^{q, p}$, this implies that $\varphi(\bar{s})=n-\varphi(s)$, and hence that $\varphi(s)+\varphi(\bar{s})=n$ for every $s \in S$.

Definition 63. Let $E$ be a number field, and $S=\operatorname{Hom}(E, \mathbb{C})$ the set of its complex embeddings. Any function $\varphi: S \rightarrow \mathbb{Z}$ with the property that $\varphi(s)+\varphi(\bar{s})=n$ defines a Hodge structure $E_{\varphi}$ of weight $n$ on the $\mathbb{Q}$-vector space $E$, whose Hodge decomposition is given by

$$
E_{\varphi} \otimes_{\mathbb{Q}} \mathbb{C} \simeq \bigoplus_{s \in S} \mathbb{C}^{\varphi(s), \varphi(\bar{s})} .
$$

By construction, the action of $E$ on itself respects this decomposition.

In summary, we have $V \simeq E_{\varphi}$, which is an isomorphism both of $E$-modules and of Hodge structures of weight $n$. Next, we would like to prove that in all interesting cases, $E$ must be a CM-field. Recall that a field $E$ is called a $C M$-field if there exists a nontrivial involution $\iota: E \rightarrow E$, such that complex conjugation induces $\iota$ under any embedding of $E$ into the complex numbers. In other words, we must have $s(\iota e)=\bar{s}(e)$ for any $s \in S$ and any $e \in E$. We usually write $\bar{e}$ in place of $\iota e$, and refer to it as complex conjugation on $E$. The fixed field of $E$ is then a totally real subfield $F$, and $E$ is a purely imaginary quadratic extension of $F$.

To prove that $E$ is either a CM-field or $\mathbb{Q}$, we choose a polarization $\psi$ on $E_{\varphi}$. We then define the so-called Rosati involution $\iota: E \rightarrow E$ by the condition that

$$
\psi(e \cdot x, y)=\psi(x, \iota e \cdot y)
$$

for every $x, y, e \in E$. Denoting the image of $1 \in E$ by $\sum_{s \in S} 1_{s}$, we have

$$
\sum_{s \in S} \psi\left(1_{s}, 1_{\bar{s}}\right) s(e \cdot x) \bar{s}(y)=\sum_{s \in S} \psi\left(1_{s}, 1_{\bar{s}}\right) s(x) \bar{s}(\iota e \cdot y),
$$

which implies that $s(e)=\bar{s}(\iota e)$. Now there are two cases: Either $\iota$ is nontrivial, in which case $E$ is a CM-field and the Rosati involution is complex conjugation. Or $\iota$ is trivial, which means that $\bar{s}=s$ for every complex embedding. In the second case, we see that $\varphi(s)=n / 2$ for every $s$, and so the Hodge structure must be $\mathbb{Q}(-n / 2)$, being irreducible and of type $(n / 2, n / 2)$. This implies that $E=\mathbb{Q}$. 
From now on, we exclude the trivial case $V=\mathbb{Q}(-n / 2)$ and assume that $E$ is a CM-field.

Definition 64. A CM-type of $E$ is a mapping $\varphi: S \rightarrow\{0,1\}$ with the property that $\varphi(s)+\varphi(\bar{s})=1$ for every $s \in S$.

When $\varphi$ is a CM-type, $E_{\varphi}$ is the rational Hodge structure of an abelian variety with complex multiplication by $E$ (unique up to isogeny). In general, we have the following structure theorem.

Proposition 65. Any Hodge structure $V$ of $C M$-type and of even weight $2 k$ with $V^{p, q}=0$ for $p<0$ or $q<0$ occurs as a direct factor of $H^{2 k}(A)$ for some abelian variety with complex multiplication.

Proof. In our classification of irreducible Hodge structures of CM-type above, there were two cases: $\mathbb{Q}(-n / 2)$, and Hodge structures of the form $E_{\varphi}$, where $E$ is a CM-field and $\varphi: S \rightarrow \mathbb{Z}$ is a function satisfying $\varphi(s)+\varphi(\bar{s})=n$. Clearly $\varphi$ can be written as a linear combination (with integer coefficients) of CM-types for $E$. Because of the relations

$$
E_{\varphi+\psi} \simeq E_{\varphi} \otimes_{E} E_{\psi} \quad \text { and } \quad E_{-\varphi} \simeq E_{\varphi}^{\vee}
$$

every irreducible Hodge structure of CM-type can thus be obtained from Hodge structures corresponding to CM-types by tensor products, duals, and Tate twists.

As we have seen, every Hodge structure of CM-type is a direct sum of irreducible Hodge structures of CM-type. The assertion follows from this by simple linear algebra.

To conclude our discussion of Hodge structures of CM-type, we will consider the case when the CM-field $E$ is a Galois extension of $\mathbb{Q}$. In that case, the Galois group $G=\operatorname{Gal}(E / \mathbb{Q})$ acts on the set of complex embeddings of $E$ by the rule

$$
(g \cdot s)(e)=s\left(g^{-1} e\right) .
$$

This action is simply transitive. Recall that we have an isomorphism

$$
E \otimes_{\mathbb{Q}} E \stackrel{\sim}{\rightarrow} \bigoplus_{g \in G} E, \quad x \otimes e \mapsto g(e) x
$$

For any $E$-vector space $V$, this isomorphism induces a decomposition

$$
V \otimes_{\mathbb{Q}} E \stackrel{\sim}{\rightarrow} \bigoplus_{g \in G} V, \quad v \otimes e \mapsto g(e) v
$$

When $V$ is an irreducible Hodge structure of CM-type, a natural question is whether this decomposition is compatible with the Hodge decomposition. The following lemma shows that the answer to this question is yes.

Lemma 66. Let $E$ be a $C M$-field that is a Galois extension of $\mathbb{Q}$, with Galois group $G=\operatorname{Gal}(E / \mathbb{Q})$. Then for any $\varphi: S \rightarrow \mathbb{Z}$ with $\varphi(s)+\varphi(\bar{s})=n$, we have

$$
E_{\varphi} \otimes_{\mathbb{Q}} E \simeq \bigoplus_{g \in G} E_{g \varphi} .
$$

Proof. We chase the Hodge decompositions through the various isomorphisms that are involved in the statement. To begin with, we have

$$
\left(E_{\varphi} \otimes_{\mathbb{Q}} E\right) \otimes_{\mathbb{Q}} \mathbb{C} \simeq\left(E_{\varphi} \otimes_{\mathbb{Q}} \mathbb{C}\right) \otimes_{\mathbb{Q}} E \simeq \bigoplus_{s \in S} \mathbb{C}^{\varphi(s), n-\varphi(s)} \otimes_{\mathbb{Q}} E \simeq \bigoplus_{s, t \in S} \mathbb{C}^{\varphi(s), n-\varphi(s)},
$$


and the isomorphism takes $(v \otimes e) \otimes z$ to the element

$$
\sum_{s, t \in S} t(e) \cdot z \cdot s(v)
$$

On the other hand,

$$
\left(E_{\varphi} \otimes_{\mathbb{Q}} E\right) \otimes_{\mathbb{Q}} \mathbb{C} \simeq \bigoplus_{g \in G} E \otimes_{\mathbb{Q}} \mathbb{C} \simeq \bigoplus_{g \in G} \bigoplus_{s \in S} \mathbb{C}^{\varphi(s), n-\varphi(s)}
$$

and under this isomorphism, $(v \otimes e) \otimes z$ is sent to the element

$$
\sum_{g \in G} \sum_{s \in S} s(g e) \cdot s(v) \cdot z .
$$

If we fix $g \in G$ and compare the two expressions, we see that $t=s g$, and hence

$$
E \otimes_{\mathbb{Q}} \mathbb{C} \simeq \bigoplus_{t \in S} \mathbb{C}^{\varphi(s), n-\varphi(s)} \simeq \bigoplus_{t \in S} \mathbb{C}^{\varphi\left(t g^{-1}\right), n-\varphi\left(t g^{-1}\right)} .
$$

But since $(g \varphi)(t)=\varphi\left(t g^{-1}\right)$, this is exactly the Hodge decomposition of $E_{g \varphi}$.

5.3. Reduction to abelian varieties of CM-type. The proof of Deligne's theorem involves the construction of algebraic families of abelian varieties, in order to apply Principle B. For this, we shall use the existence of a fine moduli space for polarized abelian varieties with level structure. Recall that if $A$ is an abelian variety of dimension $g$, the subgroup $A[N]$ of its $N$-torsion points is isomorphic to $(\mathbb{Z} / N \mathbb{Z})^{\oplus 2 g}$. A level $N$-structure is a choice of symplectic isomorphism $A[N] \simeq(\mathbb{Z} / N \mathbb{Z})^{\oplus 2 g}$. Also recall that a polarization of degree $d$ on an abelian variety $A$ is a finite morphism $\theta: A \rightarrow \hat{A}$ of degree $d$.

Theorem 67. Fix integers $g, d \geq 1$. Then for any $N \geq 3$, there is a smooth quasi-projective variety $\mathcal{M}_{g, d, N}$ that is a fine moduli space for $g$-dimensional abelian varieties with polarization of degree $d$ and level $N$-structure. In particular, we have a universal family of abelian varieties over $\mathcal{M}_{g, d, N}$.

The relationship of this result with Hodge theory is the following. Fix an abelian variety $A$ of dimension $g$, with level $N$-structure and polarization $\theta: A \rightarrow \hat{A}$. The polarization corresponds to an antisymmetric bilinear form $\psi: H^{1}(A, \mathbb{Z}) \times H^{1}(A, \mathbb{Z}) \rightarrow \mathbb{Z}$ that polarizes the Hodge structure; we shall refer to $\psi$ as a Riemann form. Define $V=H^{1}(A, \mathbb{Q})$, and let $D$ be the corresponding period domain; $D$ parametrizes all possible Hodge structures of type $\{(1,0),(0,1)\}$ on $V$ that are polarized by the form $\psi$. Then $D$ is isomorphic to the universal covering space of the quasi-projective complex manifold $\mathcal{M}_{g, d, N}$.

We now turn to the first step in the proof of Deligne's theorem, namely the reduction of the general problem to abelian varieties of CM-type. This is accomplished by the following theorem and Principle B, see Theorem 36 .

Theorem 68. Let $A$ be an abelian variety, and let $\alpha \in H^{2 p}(A, \mathbb{Q}(p))$ be a Hodge class on $A$. Then there exists a family $\pi: \mathcal{A} \rightarrow B$ of abelian varieties, with $B$ irreducible and quasi-projective, such that the following three things are true:

(a) $\mathcal{A}_{0}=A$ for some point $0 \in B$.

(b) There is a Hodge class $\tilde{\alpha} \in H^{2 p}(\mathcal{A}, \mathbb{Q}(p))$ whose restriction to $A$ equals $\alpha$.

(c) For a dense set of $t \in B$, the abelian variety $\mathcal{A}_{t}=\pi^{-1}(t)$ is of CM-type. 
Before giving the proof, we shall briefly recall the following useful interpretation for period domains. Say $D$ parametrizes all Hodge structures of weight $n$ on a fixed rational vector space $V$ that are polarized by a given bilinear form $\psi$. The set of real points of the group $G=\operatorname{Aut}(V, \psi)$ then acts transitively on $D$ by the rule $(g H)^{p, q}=g \cdot H^{p, q}$, and so $D \simeq G(\mathbb{R}) / K$.

Now points of $D$ are in one-to-one correspondence with homomorphisms of real algebraic groups $h: U(1) \rightarrow G_{\mathbb{R}}$, and we denote the Hodge structure corresponding to $h$ by $V_{h}$. Then $V_{h}^{p, q}$ is exactly the subspace of $V \otimes_{\mathbb{Q}} \mathbb{C}$ on which $h(z)$ acts as multiplication by $z^{p-q}$, and from this, it is easy to verify that $g V_{h}=V_{g h g^{-1}}$. In other words, the points of $D$ can be thought of as conjugacy classes of a fixed $h$ under the action of $G(\mathbb{R})$.

Proof of Theorem 68, After choosing a polarization $\theta: A \rightarrow \hat{A}$, we may assume that the Hodge structure on $V=H^{1}(A, \mathbb{Q})$ is polarized by a Riemann form $\psi$. Let $G=\operatorname{Aut}(V, \psi)$, and recall that $M=\mathrm{MT}(A)$ is the smallest $\mathbb{Q}$-algebraic subgroup of $G$ whose set of real points $M(\mathbb{R})$ contains the image of the homomorphism $h: U(1) \rightarrow G(\mathbb{R})$. Let $D$ be the period domain whose points parametrize all possible Hodge structures of type $\{(1,0),(0,1)\}$ on $V$ that are polarized by the form $\psi$. With $V_{h}=H^{1}(A)$ as the base point, we then have $D \simeq G(\mathbb{R}) / K$; the points of $D$ are thus exactly the Hodge structures $V_{g h g^{-1}}$, for $g \in G(\mathbb{R})$ arbitrary.

The main idea of the proof is to consider the Mumford-Tate domain

$$
D_{h}=M(\mathbb{R}) / K \cap M(\mathbb{R}) \hookrightarrow D .
$$

By definition, $D_{h}$ consists of all Hodge structures of the form $V_{g h g^{-1}}$, for $g \in M(\mathbb{R})$. As explained in Griffiths' lectures, these are precisely the Hodge structures whose Mumford-Tate group is contained in $M$.

To find Hodge structures of CM-type in $D_{h}$, we appeal to a result by Borel. Since the image of $h$ is abelian, it is contained in a maximal torus $T$ of the real Lie group $M(\mathbb{R})$. One can show that, for a generic element $\xi$ in the Lie algebra $\mathfrak{m}_{\mathbb{R}}$, this torus is the stabilizer of $\xi$ under the adjoint action by $M(\mathbb{R})$. Now $\mathfrak{m}$ is defined over $\mathbb{Q}$, and so there exist arbitrarily small elements $g \in M(\mathbb{R})$ for which $\operatorname{Ad}(g) \xi=g \xi g^{-1}$ is rational. The stabilizer $g T g^{-1}$ of such a rational point is then a maximal torus in $M$ that is defined over $\mathbb{Q}$. The Hodge structure $V_{g h g^{-1}}$ is a point of the Mumford-Tate domain $D_{h}$, and by definition of the Mumford-Tate group, we have $\mathrm{MT}\left(V_{g h g^{-1}}\right) \subseteq T$. In particular, $V_{g h g^{-1}}$ is of CM-type, because its Mumford-Tate group is abelian. This reasoning shows that $D_{h}$ contains a dense set of points of CM-type.

To obtain an algebraic family of abelian varieties with the desired properties, we can now argue as follows. Let $\mathcal{M}$ be the moduli space of abelian varieties of $\operatorname{dimension} \operatorname{dim} A$, with polarization of the same type as $\theta$, and level 3 -structure. Then $\mathcal{M}$ is a smooth quasi-projective variety, and since it is a fine moduli space, it carries a universal family. Now there are finitely many Hodge tensors $\tau_{1}, \ldots, \tau_{r}$ for $H^{1}(A)$, such that $M=\mathrm{MT}(A)$ is exactly the subgroup of $G$ fixing every $\tau_{i}$. Let $B \subseteq \mathcal{M}$ be the irreducible component of the Hodge locus of $\tau_{1}, \ldots, \tau_{r}$ that passes through the point $A$. By the theorem of Cattani-Deligne-Kaplan, $B$ is again a quasi-projective variety. Let $\pi: \mathcal{A} \rightarrow B$ be the restriction of the universal family to $B$. Then (a) is clearly satisfied for this family.

Now $D$ is the universal covering space of $\mathcal{M}$, with the point $V_{h}=H^{1}(A)$ mapping to $A$. By construction, the preimage of $B$ in $D$ is exactly the Mumford-Tate domain $D_{h}$. Indeed, consider a Hodge structure $V_{g h g^{-1}}$ in the preimage of $B$. By construction, every $\tau_{i}$ is a Hodge tensor for this Hodge structure, which shows that $\mathrm{MT}\left(V_{g h g^{-1}}\right)$ is contained in $M$. As explained above, this implies that $V_{g h g^{-1}}$ belongs to $D_{h}$. Since $D_{h}$ contains a dense set of Hodge structures of CM-type, (c) follows. Since $B$ is also contained in the Hodge locus of $\alpha$, we obtain (b) after passing to a finite cover. 
5.4. Background on hermitian forms. The second step in the proof of Deligne's theorem involves the construction of special Hodge classes on abelian varieties of CM-type, the so-called split Weil classes. This requires some background on hermitian forms, which we now provide. Throughout, $E$ is a CM-field, with totally real subfield $F$ and complex conjugation $e \mapsto \bar{e}$, and $S=\operatorname{Hom}(E, \mathbb{C})$ denotes the set of complex embeddings of $E$. An element $\zeta \in E^{\times}$is called totally imaginary if $\bar{\zeta}=-\zeta$; concretely, this means that $\bar{s}(\zeta)=-s(\zeta)$ for every complex embedding $s$. Likewise, an element $f \in F^{\times}$is said to be totally positive if $s(f)>0$ for every $s \in S$.

Definition 69. Let $V$ be an E-vector space. $A \mathbb{Q}$-bilinear form $\phi: V \times V \rightarrow E$ is said to be $E$-hermitian if $\phi(e \cdot v, w)=e \cdot \phi(v, w)$ and $\phi(v, w)=\overline{\phi(w, v)}$ for every $v, w \in V$ and every $e \in E$.

Now suppose that $V$ is an $E$-vector space of dimension $d=\operatorname{dim}_{E} V$, and that $\phi$ is an $E$ hermitian form on $V$. We begin by describing the numerical invariants of the pair $(V, \phi)$. For any embedding $s: E \hookrightarrow \mathbb{C}$, we obtain a hermitian form $\phi_{s}$ (in the usual sense) on the complex vector space $V_{s}=V \otimes_{E, s} \mathbb{C}$. We let $a_{s}$ and $b_{s}$ be the dimensions of the maximal subspaces where $\phi_{s}$ is, respectively, positive and negative definite.

A second invariant of $\phi$ is its discriminant. To define it, note that $\phi$ induces an $E$-hermitian form on the one-dimensional $E$-vector space $\bigwedge_{E}^{d} V$, which up to a choice of basis vector, is of the form $(x, y) \mapsto f x \bar{y}$. The element $f$ belongs to the totally real subfield $F$, and a different choice of basis vector only changes $f$ by elements of the form $\mathrm{Nm}_{E / F}(e)=e \cdot \bar{e}$. Consequently, the class of $f$ in $F^{\times} / \mathrm{Nm}_{E / F}\left(E^{\times}\right)$is well-defined, and is called the discriminant of $(V, \phi)$. We denote it by the symbol disc $\phi$.

Now suppose that $\phi$ is nondegenerate. Let $v_{1}, \ldots, v_{d}$ be an orthogonal basis for $V$, and set $c_{i}=\phi\left(v_{i}, v_{i}\right)$. Then we have $c_{i} \in F^{\times}$, and

$$
a_{s}=\#\left\{i \mid s\left(c_{i}\right)>0\right\} \quad \text { and } \quad b_{s}=\#\left\{i \mid s\left(c_{i}\right)<0\right\}
$$

satisfy $a_{s}+b_{s}=d$. Moreover, we have

$$
f=\prod_{i=1}^{d} c_{i} \bmod \operatorname{Nm}_{E / F}\left(E^{\times}\right) ;
$$

this implies that $\operatorname{sgn}(s(f))=(-1)^{b_{s}}$ for every $s \in S$. The following theorem by Landherr [25] shows that the discriminant and the integers $a_{s}$ and $b_{s}$ are a complete set of invariants for $E$-hermitian forms.

Theorem 70 (Landherr). Let $a_{s}, b_{s} \geq 0$ be a collection of integers, indexed by the set $S$, and let $f \in F^{\times} / \mathrm{Nm}_{E / F}\left(E^{\times}\right)$be an arbitrary element. Suppose that they satisfy $a_{s}+b_{s}=d$ and $\operatorname{sgn}(s(f))=(-1)^{b_{s}}$ for every $s \in S$. Then there exists a nondegenerate E-hermitian form $\phi$ on an E-vector space $V$ of dimension $d$ with these invariants; moreover, $(V, \phi)$ is unique up to isomorphism.

This classical result has the following useful consequence.

Corollary 71. If $(V, \phi)$ is nondegenerate, then the following two conditions are equivalent:

(a) $a_{s}=b_{s}=d / 2$ for every $s \in S$, and $\operatorname{disc} \phi=(-1)^{d / 2}$.

(b) There is a totally isotropic subspace of $V$ of dimension $d / 2$.

Proof. If $W \subseteq V$ is a totally isotropic subspace of dimension $d / 2$, then $v \mapsto \phi(-, v)$ induces an antilinear isomorphism $V / W \stackrel{\sim}{\rightarrow} W^{\vee}$. Thus we can extend a basis $v_{1}, \ldots, v_{d / 2}$ of $W$ to a basis 
$v_{1}, \ldots, v_{d}$ of $V$, with the property that

$$
\begin{aligned}
\phi\left(v_{i}, v_{i+d / 2}\right)=1 & \text { for } 1 \leq i \leq d / 2, \\
\phi\left(v_{i}, v_{j}\right)=0 & \text { for }|i-j| \neq d / 2 .
\end{aligned}
$$

We can use this basis to check that (a) is satisfied. For the converse, consider the hermitian space $\left(E^{\oplus d}, \phi\right)$, where

$$
\phi(x, y)=\sum_{1 \leq i \leq d / 2}\left(x_{i} \bar{y}_{i+d / 2}+x_{i+d / 2} \bar{y}_{i}\right)
$$

for every $x, y \in E^{\oplus d}$. By Landherr's theorem, this space is (up to isomorphism) the unique hermitian space satisfying (a), and it is easy to see that it satisfies (b), too.

Definition 72. An E-hermitian form $\phi$ that satisfies the two equivalent conditions in Corollary 71 is said to be split.

We shall see below that $E$-hermitian forms are related to polarizations on Hodge structures of CM-type. We now describe one additional technical result that shall be useful in that context. Suppose that $V$ is a Hodge structure of type $\{(1,0),(0,1)\}$ that is of CM-type and whose endomorphism ring contains $E$; let $h: U(1) \rightarrow E^{\times}$be the corresponding homomorphism. Recall that a Riemann form for $V$ is a $\mathbb{Q}$-bilinear antisymmetric form $\psi: V \times V \rightarrow \mathbb{Q}$, with the property that

$$
(x, y) \mapsto \psi(x, h(i) \cdot \bar{y})
$$

is hermitian and positive definite on $V \otimes_{\mathbb{Q}} \mathbb{C}$. We only consider Riemann forms whose Rosati involution induces complex conjugation on $E$; that is, which satisfy

$$
\psi(e v, w)=\psi(v, \bar{e} w) .
$$

Lemma 73. Let $\zeta \in E^{\times}$be a totally imaginary element $(\bar{\zeta}=-\zeta)$, and let $\psi$ be a Riemann form for $V$ as above. Then there exists a unique E-hermitian form $\phi$ with the property that $\psi=\operatorname{Tr}_{E / \mathbb{Q}}(\zeta \phi)$.

We begin with a simpler statement.

Lemma 74. Let $V$ and $W$ be finite-dimensional vector spaces over $E$, and let $\psi: V \times W \rightarrow \mathbb{Q}$ be a $\mathbb{Q}$-bilinear form such that $\psi(e v, w)=\psi(v, e w)$ for every $e \in E$. Then there exists a unique E-bilinear form $\phi$ such that $\psi(v, w)=\operatorname{Tr}_{E / \mathbb{Q}} \phi(v, w)$.

Proof. The trace pairing $E \times E \rightarrow \mathbb{Q},(x, y) \mapsto \operatorname{Tr}_{E / \mathbb{Q}}(x y)$, is nondegenerate. Consequently, composition with $\operatorname{Tr}_{E / \mathbb{Q}}$ induces an injective homomorphism

$$
\operatorname{Hom}_{E}\left(V \otimes_{E} W, E\right) \rightarrow \operatorname{Hom}_{\mathbb{Q}}\left(V \otimes_{E} W, \mathbb{Q}\right),
$$

which has to be an isomorphism because both vector spaces have the same dimension over $\mathbb{Q}$. By assumption, $\psi$ defines a $\mathbb{Q}$-linear map $V \otimes_{E} W \rightarrow \mathbb{Q}$, and we let $\phi$ be the element of $\operatorname{Hom}_{E}\left(V \otimes_{E}\right.$ $W, E)$ corresponding to $\psi$ under the above isomorphism.

Proof of Lemma 73. We apply the preceding lemma with $W=V$, but with $E$ acting on $W$ through complex conjugation. This gives a sesquilinear form $\phi_{1}$ such that $\psi(x, y)=\operatorname{Tr}_{E / \mathbb{Q}} \phi_{1}(x, y)$. Now define $\phi=\zeta^{-1} \phi_{1}$, so that we have $\psi(x, y)=\operatorname{Tr}_{E / \mathbb{Q}}(\zeta \phi(x, y))$. The uniqueness of $\phi$ is obvious from the preceding lemma.

It remains to show that we have $\phi(y, x)=\overline{\phi(x, y)}$. Because $\psi$ is antisymmetric, $\psi(y, x)=$ $-\psi(x, y)$, which implies that

$$
\operatorname{Tr}_{E / \mathbb{Q}}(\zeta \phi(y, x))=-\operatorname{Tr}_{E / \mathbb{Q}}(\zeta \phi(x, y))=\operatorname{Tr}_{E / \mathbb{Q}}(\bar{\zeta} \phi(x, y)) .
$$


On replacing $y$ by $e y$, for arbitrary $e \in E$, we obtain

$$
\operatorname{Tr}_{E / \mathbb{Q}}(\zeta e \cdot \phi(y, x))=\operatorname{Tr}_{E / \mathbb{Q}}(\overline{\zeta e} \cdot \phi(x, y)) .
$$

On the other hand, we have

$$
\operatorname{Tr}_{E / \mathbb{Q}}(\zeta e \cdot \phi(y, x))=\operatorname{Tr}_{E / \mathbb{Q}}(\overline{\zeta e \cdot \phi(y, x)})=\operatorname{Tr}_{E / \mathbb{Q}}(\overline{\zeta e} \cdot \overline{\phi(y, x)}) .
$$

Since $\overline{\zeta e}$ can be an arbitrary element of $E$, the nondegeneracy of the trace pairing implies that $\phi(x, y)=\overline{\phi(y, x)}$.

5.5. Construction of split Weil classes. Let $E$ be a CM-field; as usual, we let $S=\operatorname{Hom}(E, \mathbb{C})$ be the set of complex embeddings; it has $[E: \mathbb{Q}]$ elements.

Let $V$ be a rational Hodge structure of type $\{(1,0),(0,1)\}$ whose endomorphism algebra contains $E$. We shall assume that $\operatorname{dim}_{E} V=d$ is an even number. Let $V_{s}=V \otimes_{E, s} \mathbb{C}$. Corresponding to the decomposition

$$
E \otimes \mathbb{Q} \mathbb{C} \stackrel{\sim}{\rightarrow} \bigoplus_{s \in S} \mathbb{C}, \quad e \otimes z \mapsto \sum_{s \in S} s(e) z,
$$

we get a decomposition

$$
V \otimes_{\mathbb{Q}} \mathbb{C} \simeq \bigoplus_{s \in S} V_{s}
$$

The isomorphism is $E$-linear, where $e \in E$ acts on the complex vector space $V_{s}$ as multiplication by $s(e)$. Since $\operatorname{dim}_{\mathbb{Q}} V=[E: \mathbb{Q}] \cdot \operatorname{dim}_{E} V$, each $V_{s}$ has dimension $d$ over $\mathbb{C}$. By assumption, $E$ respects the Hodge decomposition on $V$, and so we get an induced decomposition

$$
V_{s}=V_{s}^{1,0} \oplus V_{s}^{0,1} \text {. }
$$

Note that $\operatorname{dim}_{\mathbb{C}} V_{s}^{1,0}+\operatorname{dim}_{\mathbb{C}} V_{s}^{0,1}=d$.

Lemma 75. The rational subspace $\bigwedge_{E}^{d} V \subseteq \bigwedge_{\mathbb{Q}}^{d} V$ is purely of type $(d / 2, d / 2)$ if and only if $\operatorname{dim}_{\mathbb{C}} V_{s}^{1,0}=\operatorname{dim}_{\mathbb{C}} V_{s}^{0,1}=d / 2$ for every $s \in S$.

Proof. We have

$$
\left(\bigwedge_{E}^{d} V\right) \otimes_{\mathbb{Q}} \mathbb{C} \simeq \bigwedge_{E \otimes_{\mathbb{C}}}^{d} V \otimes_{\mathbb{Q}} \mathbb{C} \simeq \bigoplus_{s \in S} \bigwedge_{\mathbb{C}}^{d} V_{s} \simeq \bigoplus_{s \in S}\left(\bigwedge_{\mathbb{C}}^{p_{s}} V_{s}^{1,0}\right) \otimes\left(\bigwedge_{\mathbb{C}}^{q_{s}} V_{s}^{0,1}\right),
$$

where $p_{s}=\operatorname{dim}_{\mathbb{C}} V_{s}^{1,0}$ and $q_{s}=\operatorname{dim}_{\mathbb{C}} V_{s}^{0,1}$. The assertion follows because the Hodge type of each summand is evidently $\left(p_{s}, q_{s}\right)$.

We will now describe a condition on $V$ that guarantees that the space $\bigwedge_{E}^{d} V$ consists entirely of Hodge cycles.

Definition 76. Let $V$ be a rational Hodge structure of type $\{(1,0),(0,1)\}$ with $E \hookrightarrow \operatorname{End}_{\mathbb{Q}-H S}(V)$ and $\operatorname{dim}_{E} V=d$. We say that $V$ is of split Weil type relative to $E$ if there exists an E-hermitian form $\phi$ on $V$ with a totally isotropic subspace of dimension $d / 2$, and a totally imaginary element $\zeta \in E$, such that $\operatorname{Tr}_{E / \mathbb{Q}}(\zeta \phi)$ defines a polarization on $V$.

According to Corollary 71 the condition on the E-hermitian form $\phi$ is the same as saying that the pair $(V, \phi)$ is split. 
Proposition 77. If $V$ is of split Weil type relative to $E$, and $\operatorname{dim}_{E} V=d$ is even, then the space

$$
\bigwedge_{E}^{d} V \subseteq \bigwedge_{\mathbb{Q}}^{d} V
$$

consists of Hodge classes of type $(d / 2, d / 2)$.

Proof. Since $\psi=\operatorname{Tr}_{E / \mathbb{Q}}(\zeta \phi)$ defines a polarization, $\phi$ is nondegenerate; by Corollary 71, it follows that $(V, \phi)$ is split. Thus for any complex embedding $s: E \hookrightarrow \mathbb{C}$, we have $a_{s}=b_{s}=d / 2$. Let $\phi_{s}$ be the induced hermitian form on $V_{s}=V \otimes_{E, s} \mathbb{C}$. By Lemma 75, it suffices to show that $\operatorname{dim}_{\mathbb{C}} V_{s}^{1,0}=\operatorname{dim}_{\mathbb{C}} V_{s}^{0,1}=d / 2$. By construction, the isomorphism

$$
\alpha: V \otimes_{\mathbb{Q}} \mathbb{C} \stackrel{\sim}{\rightarrow} \bigoplus_{s \in S} V_{s}
$$

respects the Hodge decompositions on both sides. For any $v \in V$, we have

$$
\psi(v, v)=\operatorname{Tr}_{E / \mathbb{Q}}(\zeta \phi(v, v))=\sum_{s \in S} s(\zeta) \cdot s(\phi(v, v))=\sum_{s \in S} s(\zeta) \cdot \phi_{s}(v \otimes 1, v \otimes 1) .
$$

Now if we choose a nonzero element $x \in V_{s}^{1,0}$, then under the above isomorphism,

$$
-s(\zeta) i \cdot \phi_{s}(x, \bar{x})=\psi\left(\alpha^{-1}(x), h(i) \cdot \overline{\alpha^{-1}(x)}\right)>0
$$

Likewise, we have $s(\zeta) i \cdot \phi_{s}(x, \bar{x})>0$ for $x \in V_{s}^{0,1}$ nonzero. Consequently, $\operatorname{dim}_{\mathbb{C}} V_{s}^{1,0}$ and $\operatorname{dim}_{\mathbb{C}} V_{s}^{0,1}$ must both be less than or equal to $d / 2=a_{s}=b_{s}$; since their dimensions add up to $d$, we get the desired result.

5.6. André's theorem and reduction to split Weil classes. The second step in the proof of Deligne's theorem is to reduce the problem from arbitrary Hodge classes on abelian varieties of CM-type to Hodge classes of split Weil type. This is accomplished by the following pretty theorem due to Yves André in [2].

Theorem 78 (André). Let $V$ be a rational Hodge structure of type $\{(1,0),(0,1)\}$, which is of $C M$ type. Then there exists a CM-field E, rational Hodge structures $V_{\alpha}$ of split Weil type (relative to $E)$, and morphisms of Hodge structure $V_{\alpha} \rightarrow V$, such that every Hodge cycle $\xi \in \bigwedge_{\mathbb{Q}}^{2 k} V$ is a sum of images of Hodge cycles $\xi_{\alpha} \in \bigwedge_{\mathbb{Q}}^{2 k} V_{\alpha}$ of split Weil type.

Proof. Let $V=V_{1} \oplus \cdots \oplus V_{r}$, with $V_{i}$ irreducible; then each $E_{i}=\operatorname{End}_{\mathbb{Q}-\mathrm{HS}}\left(V_{i}\right)$ is a CM-field. Define $E$ to be the Galois closure of the compositum of the fields $E_{1}, \ldots, E_{r}$, so that $E$ is a CM-field which is Galois over $\mathbb{Q}$ with Galois group $G=\operatorname{Gal}\left(E / \mathbb{Q}\right.$ ). After replacing $V$ by $V \otimes_{\mathbb{Q}} E$ (of which $V$ is a direct factor), we may assume without loss of generality that $E_{i}=E$ for all $i$.

As before, let $S=\operatorname{Hom}(E, \mathbb{C})$ be the set of complex embeddings of $E$; we then have a decomposition

$$
V \simeq \bigoplus_{i \in I} E_{\varphi_{i}}
$$

for some collection of CM-types $\varphi_{i}$. Applying Lemma 66 we get

$$
V \otimes_{\mathbb{Q}} E \simeq \bigoplus_{i \in I} \bigoplus_{g \in G} E_{g \varphi_{i}} .
$$

Since each $E_{g \varphi_{i}}$ is one-dimensional over $E$, we get

$$
\left(\bigwedge_{\mathbb{Q}}^{2 k} V\right) \otimes_{\mathbb{Q}} E \simeq \bigwedge_{E}^{2 k} V \otimes_{\mathbb{Q}} E \simeq \bigwedge_{E}^{2 k} \bigoplus_{(i, g) \in I \times G} E_{g \varphi_{i}} \simeq \bigoplus_{\substack{\alpha \subseteq I \times G(i, g) \in \alpha \\|\bar{\alpha}|=2 k}} \bigotimes_{g \varphi_{i}}
$$


where the tensor product is over $E$. If we now define Hodge structures of CM-type

$$
V_{\alpha}=\bigoplus_{(i, g) \in \alpha} E_{g \varphi_{i}}
$$

for any subset $\alpha \subseteq I \times G$ of size $2 k$, then $V_{\alpha}$ has dimension $2 k$ over $E$. The above calculation shows that

$$
\left(\bigwedge_{\mathbb{Q}}^{2 k} V\right) \otimes_{\mathbb{Q}} E \simeq \bigoplus_{\alpha} \bigwedge_{E}^{2 k} V_{\alpha}
$$

which is an isomorphism both as Hodge structures and as $E$-vector spaces. Moreover, since $V_{\alpha}$ is a sub-Hodge structure of $V \otimes_{\mathbb{Q}} E$, we clearly have morphisms $V_{\alpha} \rightarrow V$, and any Hodge cycle $\xi \in \bigwedge_{\mathbb{Q}}^{2 k} V$ is a sum of Hodge cycles $\xi_{\alpha} \in \bigwedge_{E}^{2 k} V_{\alpha}$.

It remains to see that $V_{\alpha}$ is of split Weil type whenever $\xi_{\alpha}$ is nonzero. Fix a subset $\alpha \subseteq I \times G$ of size $2 k$, with the property that $\xi_{\alpha} \neq 0$. Note that we have

$$
\bigwedge_{E}^{2 k} V_{\alpha} \simeq \bigotimes_{(i, g) \in \alpha} E_{g \varphi_{i}} \simeq E_{\varphi}
$$

where $\varphi: S \rightarrow \mathbb{Z}$ is the function

$$
\varphi=\sum_{(i, g) \in \alpha} g \varphi_{i}
$$

The Hodge decomposition of $E_{\varphi}$ is given by

$$
E_{\varphi} \otimes_{\mathbb{Q}} \mathbb{C} \simeq \bigoplus_{s \in S} \mathbb{C}^{\varphi(s), \varphi(\bar{s})} .
$$

The image of the Hodge cycle $\xi_{\alpha}$ in $E_{\varphi}$ must be purely of type $(k, k)$ with respect to this decomposition. But

$$
\xi_{\alpha} \otimes 1 \mapsto \sum_{s \in S} s\left(\xi_{\alpha}\right),
$$

and since each $s\left(\xi_{\alpha}\right)$ is nonzero, we conclude that $\varphi(s)=k$ for every $s \in S$. This means that the sum of the $2 k$ CM-types $g \varphi_{i}$, indexed by $(i, g) \in \alpha$, is constant on $S$. We conclude by the criterion in Proposition 79 that $V_{\alpha}$ is of split Weil type.

The proof makes use of the following criterion for a Hodge structure to be of split Weil type. Let $\varphi_{1}, \ldots, \varphi_{d}$ be CM-types attached to $E$. Let $V_{i}=E_{\varphi_{i}}$ be the Hodge structure of CM-type corresponding to $\varphi_{i}$, and define

$$
V=\bigoplus_{i=1}^{d} V_{i} .
$$

Then $V$ is a Hodge structure of CM-type with $\operatorname{dim}_{E} V=d$.

Proposition 79. If $\sum \varphi_{i}$ is constant on $S$, then $V$ is of split Weil type.

Proof. To begin with, it is necessarily the case that $\sum \varphi_{i}=d / 2$; indeed,

$$
\sum_{i=1}^{d} \varphi_{i}(s)+\sum_{i=1}^{d} \varphi(\bar{s})=\sum_{i=1}^{d}\left(\varphi_{i}(s)+\varphi_{i}(\bar{s})\right)=d,
$$


and the two sums are equal by assumption. By construction, we have

$$
V \otimes_{\mathbb{Q}} \mathbb{C} \simeq \bigoplus_{i=1}^{d}\left(E_{\varphi_{i}} \otimes_{\mathbb{Q}} \mathbb{C}\right) \simeq \bigoplus_{i=1}^{d} \bigoplus_{s \in S} \mathbb{C}^{\varphi_{i}(s), \varphi_{i}(\bar{s})}
$$

This shows that

$$
V_{s}=V \otimes_{E, s} \mathbb{C} \simeq \bigoplus_{i=1}^{d} \mathbb{C}^{\varphi_{i}(s), \varphi_{i}(\bar{s})}
$$

Therefore $\operatorname{dim}_{\mathbb{C}} V_{s}^{1,0}=\sum \varphi_{i}(s)=d / 2$, and likewise $\operatorname{dim}_{\mathbb{C}} V_{s}^{0,1}=\sum \varphi_{i}(\bar{s})=d / 2$.

Next, we construct the required $E$-hermitian form on $V$. For each $i$, choose a Riemann form $\psi_{i}$ on $V_{i}$, whose Rosati involution acts as complex conjugation on $E$. Since $V_{i}=E_{\varphi_{i}}$, there exist totally imaginary elements $\zeta_{i} \in E^{\times}$, such that

$$
\psi_{i}(x, y)=\operatorname{Tr}_{E / \mathbb{Q}}\left(\zeta_{i} x \bar{y}\right)
$$

for every $x, y \in E$. Set $\zeta=\zeta_{d}$, and define $\phi_{i}(x, y)=\zeta_{i} \zeta^{-1} x \bar{y}$, which is an $E$-hermitian form on $V_{i}$ with the property that $\psi_{i}=\operatorname{Tr}_{E / \mathbb{Q}}\left(\zeta \phi_{i}\right)$.

For any collection of totally positive elements $f_{i} \in F$,

$$
\psi=\sum_{i=1}^{d} f_{i} \psi_{i}
$$

is a Riemann form for $V$. As $E$-vector spaces, we have $V=E^{\oplus d}$, and so we can define a nondegenerate $E$-hermitian form on $V$ by the rule

$$
\phi(v, w)=\sum_{i=1}^{d} f_{i} \phi_{i}\left(v_{i}, w_{i}\right) .
$$

We then have $\psi=\operatorname{Tr}_{E / \mathbb{Q}}(\zeta \phi)$. By the same argument as before, $a_{s}=b_{s}=d / 2$, since $\operatorname{dim}_{\mathbb{C}} V_{s}^{1,0}=$ $\operatorname{dim}_{\mathbb{C}} V_{s}^{0,1}=d / 2$. By construction, the form $\phi$ is diagonalized, and so its discriminant is easily found to be

$$
\operatorname{disc} \phi=\zeta^{-d} \prod_{i=1}^{d} f_{i} \zeta_{i} \bmod \mathrm{Nm}_{E / F}\left(E^{\times}\right) .
$$

On the other hand, we know from general principles that, for any $s \in S$,

$$
\operatorname{sgn}(s(\operatorname{disc} \phi))=(-1)^{b_{s}}=(-1)^{d / 2} .
$$

This means that $\operatorname{disc} \phi=(-1)^{d / 2} f$ for some totally positive element $f \in F^{\times}$. Upon replacing $f_{d}$ by $f_{d} f^{-1}$, we get $\operatorname{disc} \phi=(-1)^{d / 2}$, which proves that $(V, \phi)$ is split.

5.7. Split Weil classes are absolute. The third step in the proof of Deligne's theorem is to show that split Weil classes are absolute. We begin by describing a special class of abelian varieties of split Weil type where this can be proved directly.

Let $V_{0}$ be a rational Hodge structure of even rank $d$ and type $\{(1,0),(0,1)\}$. Let $\psi_{0}$ be a Riemann form that polarizes $V_{0}$, and $W_{0}$ a maximal isotropic subspace of dimension $d / 2$. Also fix an element $\zeta \in E^{\times}$with $\bar{\zeta}=-\zeta$.

Now set $V=V_{0} \otimes_{\mathbb{Q}} E$, with Hodge structure induced by the isomorphism

$$
V \otimes_{\mathbb{Q}} \mathbb{C} \simeq V_{0} \otimes_{\mathbb{Q}}\left(E \otimes_{\mathbb{Q}} \mathbb{C}\right) \simeq \bigoplus_{s \in S} V_{0} \otimes_{\mathbb{Q}} \mathbb{C}
$$


Define a $\mathbb{Q}$-bilinear form $\psi: V \times V \rightarrow \mathbb{Q}$ by the formula

$$
\psi\left(v_{0} \otimes e, v_{0}^{\prime} \otimes e^{\prime}\right)=\operatorname{Tr}_{E / \mathbb{Q}}\left(e \overline{e^{\prime}}\right) \cdot \psi_{0}\left(v_{0}, v_{0}^{\prime}\right) .
$$

This is a Riemann form on $V$, for which $W=W_{0} \otimes_{\mathbb{Q}} E$ is an isotropic subspace of dimension $d / 2$. By Lemma 73, there is a unique $E$-hermitian form $\phi: V \times V \rightarrow E$ such that $\psi=\operatorname{Tr}_{E / \mathbb{Q}}(\zeta \phi)$. By Corollary 71 ( $(V, \phi)$ is split, and $V$ is therefore of split Weil type. Let $A_{0}$ be an abelian variety with $H^{1}\left(A_{0}, \mathbb{Q}\right)=V_{0}$. The integral lattice of $V_{0}$ induces an integral lattice in $V=V_{0} \otimes_{\mathbb{Q}} E$. We denote by $A_{0} \otimes_{\mathbb{Q}} E$ the corresponding abelian variety. It is of split Weil type since $V$ is.

The next result, albeit elementary, is the key to proving that split Weil classes are absolute.

Proposition 80. Let $A_{0}$ be an abelian variety with $H^{1}\left(A_{0}\right)=V_{0}$, and define $A=A_{0} \otimes_{\mathbb{Q}} E$. Then the subspace $\bigwedge_{E}^{d} H^{1}(A, \mathbb{Q})$ of $H^{d}(A, \mathbb{Q})$ consists entirely of absolute Hodge classes.

Proof. We have $H^{d}(A, \mathbb{Q}) \simeq \bigwedge_{\mathbb{Q}}^{d} H^{1}(A, \mathbb{Q})$, and the subspace

$$
\bigwedge_{E}^{d} H^{1}(A, \mathbb{Q}) \simeq \bigwedge_{E}^{d} V_{0} \otimes_{\mathbb{Q}} E \simeq\left(\bigwedge_{\mathbb{Q}}^{d} V_{0}\right) \otimes_{\mathbb{Q}} E \simeq H^{d}\left(A_{0}, \mathbb{Q}\right) \otimes_{\mathbb{Q}} E
$$

consists entirely of Hodge classes by Proposition 77 But since $\operatorname{dim} A_{0}=d / 2$, the space $H^{d}\left(A_{0}, \mathbb{Q}\right)$ is generated by the fundamental class of a point, which is clearly absolute. This implies that every class in $\bigwedge_{E}^{d} H^{1}(A, \mathbb{Q})$ is absolute.

The following theorem, together with Principle B as in Theorem 36 completes the proof of Deligne's theorem.

Theorem 81. Let $E$ be a $C M$-field, and let $A$ be an abelian variety of split Weil type (relative to $E$ ). Then there exists a family $\pi: \mathcal{A} \rightarrow B$ of abelian varieties, with $B$ irreducible and quasi-projective, such that the following three things are true:

(a) $\mathcal{A}_{0}=A$ for some point $0 \in B$.

(b) For every $t \in B$, the abelian variety $\mathcal{A}_{t}=\pi^{-1}(t)$ is of split Weil type (relative to $E$ ).

(c) The family contains an abelian variety of the form $A_{0} \otimes_{\mathbb{Q}} E$.

The proof of Theorem 81 takes up the remainder of this section. Throughout, we let $V=$ $H^{1}(A, \mathbb{Q})$, which is an $E$-vector space of some even dimension $d$. The polarization on $A$ corresponds to a Riemann form $\psi: V \times V \rightarrow \mathbb{Q}$, with the property that the Rosati involution acts as complex conjugation on $E$. Fix a totally imaginary element $\zeta \in E^{\times}$; then $\psi=\operatorname{Tr}_{E / \mathbb{Q}}(\zeta \phi)$ for a unique $E$-hermitian form $\phi$ by Lemma 73. Since $A$ is of split Weil type, the pair $(V, \phi)$ is split.

As before, let $D$ be the period domain, whose points parametrize Hodge structures of type $\{(1,0),(0,1)\}$ on $V$ that are polarized by the form $\psi$. Let $D^{\text {sp }} \subseteq D$ be the subset of those Hodge structures that are of split Weil type (relative to $E$, and with polarization given by $\psi$ ). We shall show that $D^{\mathrm{sp}}$ is a certain hermitian symmetric domain.

We begin by observing that there are essentially $2^{[E: \mathbb{Q}]} / 2$ many different choices for the totally imaginary element $\zeta$, up to multiplication by totally positive elements in $F^{\times}$. Indeed, if we fix a choice of $i=\sqrt{-1}$, and define $\varphi_{\zeta}: S \rightarrow\{0,1\}$ by the rule

$$
\varphi_{\zeta}(s)= \begin{cases}1 & \text { if } s(\zeta) i>0, \\ 0 & \text { if } s(\zeta) i<0,\end{cases}
$$

then $\varphi_{\zeta}(s)+\varphi_{\zeta}(\bar{s})=1$ because $\bar{s}(\zeta)=-s(\zeta)$, and so $\varphi_{\zeta}$ is a CM-type for $E$. Conversely, one can show that any CM-type is obtained in this manner. 
Lemma 82. The subset $D^{\mathrm{sp}}$ of the period domain $D$ is a hermitian symmetric domain; in fact, it is isomorphic to the product of $|S|=[E: \mathbb{Q}]$ many copies of Siegel upper halfspace.

Proof. Recall that $V$ is an $E$-vector space of even dimension $d$, and that the Riemann form $\psi=$ $\operatorname{Tr}_{E / \mathbb{Q}}(\zeta \phi)$ for a split $E$-hermitian form $\phi: V \times V \rightarrow E$ and a totally imaginary $\zeta \in E^{\times}$. The Rosati involution corresponding to $\psi$ induces complex conjugation on $E$; this means that $\psi(e v, w)=$ $\psi(v, \bar{e} w)$ for every $e \in E$.

By definition, $D^{\text {sp }}$ parametrizes all Hodge structures of type $\{(1,0),(0,1)\}$ on $V$ that admit $\psi$ as a Riemann form and are of split Weil type (relative to the CM-field $E$ ). Such a Hodge structure amounts to a decomposition

$$
V \otimes_{\mathbb{Q}} \mathbb{C}=V^{1,0} \oplus V^{0,1}
$$

with $V^{0,1}=\overline{V^{1,0}}$, with the following two properties:

(a) The action by $E$ preserves $V^{1,0}$ and $V^{0,1}$.

(b) The form $-i \psi(x, \bar{y})=\psi(x, h(i) \bar{y})$ is positive definite on $V^{1,0}$.

Let $S=\operatorname{Hom}(E, \mathbb{C})$, and consider the isomorphism

$$
V \otimes \mathbb{Q} \mathbb{C} \stackrel{\sim}{\rightarrow} \bigoplus_{s \in S} V_{s}, \quad v \otimes z \mapsto \sum_{s \in S} v \otimes z,
$$

where $V_{s}=V \otimes_{E, s} \mathbb{C}$. Since $V_{s}$ is exactly the subspace on which $e \in E$ acts as multiplication by $s(e)$, the condition in (a) is equivalent to demanding that each complex vector space $V_{s}$ decomposes as $V_{s}=V_{s}^{1,0} \oplus V_{s}^{0,1}$.

On the other hand, $\phi$ induces a hermitian form $\phi_{s}$ on each $V_{s}$, and we have

$$
\psi(v, w)=\operatorname{Tr}_{E / \mathbb{Q}}(\zeta \phi(v, w))=\sum_{s \in S} s(\zeta) \phi_{s}(v \otimes 1, w \otimes 1) .
$$

Therefore $\psi$ polarizes the Hodge structure $V^{1,0} \oplus V^{0,1}$ if and only if the form $x \mapsto-s(\zeta) i \cdot \phi_{s}(x, \bar{x})$ is positive definite on the subspace $V_{s}^{1,0}$. Referring to the definition of $\varphi_{\zeta}$ in (6), this is equivalent to demanding that $x \mapsto(-1)^{\varphi_{\zeta}(s)} \phi_{s}(x, \bar{x})$ be positive definite on $V_{s}^{1,0}$.

In summary, Hodge structures of split Weil type on $V$ for which $\psi$ is a Riemann form are parametrized by a choice of $d / 2$-dimensional complex subspaces $V_{s}^{1,0} \subseteq V_{s}$, one for each $s \in S$, with the property that

$$
V_{s}^{1,0} \cap \overline{V_{s}^{1,0}}=\{0\}
$$

and such that $x \mapsto(-1)^{\varphi_{\zeta}(s)} \phi_{s}(x, \bar{x})$ is positive definite on $V_{s}^{1,0}$. Since for each $s \in S$, we have $a_{s}=b_{s}=d / 2$, the hermitian form $\phi_{s}$ has signature $(d / 2, d / 2)$; this implies that the space

$$
D_{s}=\left\{W \in \operatorname{Grass}_{d / 2}\left(V_{s}\right) \mid W \cap \bar{W}=\{0\} \text { and }(-1)^{\varphi_{\zeta}(s)} \phi_{s}(x, \bar{x})>0 \text { for } 0 \neq x \in W\right\}
$$

is isomorphic to Siegel upper halfspace. The parameter space $D^{\mathrm{sp}}$ for our Hodge structures is therefore the hermitian symmetric domain

$$
D^{\mathrm{sp}} \simeq \prod_{s \in S} D_{s} .
$$

In particular, it is a connected complex manifold.

To be able to satisfy the final condition in Theorem 81, we need to know that $D^{\text {sp }}$ contains Hodge structures of the form $V_{0} \otimes_{\mathbb{Q}} E$. This is the content of the following lemma.

Lemma 83. With notation as above, there is a rational Hodge structure $V_{0}$ of weight one, such that $V_{0} \otimes_{\mathbb{Q}} E$ belongs to $D^{\mathrm{sp}}$. 
Proof. Since the pair $(V, \phi)$ is split, there is a totally isotropic subspace $W \subseteq V$ of dimension $\operatorname{dim}_{E} W=d / 2$. Arguing as in the proof of Corollary 71, we can therefore find a basis $v_{1}, \ldots, v_{d}$ for the $E$-vector space $V$, with the property that

$$
\begin{aligned}
\phi\left(v_{i}, v_{i+d / 2}\right)=\zeta^{-1} & \text { for } 1 \leq i \leq d / 2 \\
\phi\left(v_{i}, v_{j}\right)=0 & \text { for }|i-j| \neq d / 2 .
\end{aligned}
$$

Let $V_{0}$ be the $\mathbb{Q}$-linear span of $v_{1}, \ldots, v_{d}$; then we have $V=V_{0} \otimes_{\mathbb{Q}} E$. Now define $V_{0}^{1,0} \subseteq V_{0} \otimes_{\mathbb{Q}} \mathbb{C}$ as the $\mathbb{C}$-linear span of the vectors $h_{k}=v_{k}+i v_{k+d / 2}$ for $k=1, \ldots, d / 2$. Evidently, this gives Hodge structure of weight one on $V_{0}$, with hence a Hodge structure on $V=V_{0} \otimes_{\mathbb{Q}} E$. It remains to show that $\psi$ polarizes this Hodge structure. But we compute that

$$
\begin{aligned}
\psi\left(\sum_{j=1}^{d / 2} a_{j} h_{j}, i \sum_{k=1}^{d / 2} \overline{a_{k} h_{k}}\right) & =\sum_{k=1}^{d / 2}\left|a_{k}\right|^{2} \psi\left(v_{k}+i v_{k+d / 2}, i\left(v_{k}-i v_{k+d / 2}\right)\right)=2 \sum_{k=1}^{d / 2}\left|a_{k}\right|^{2} \psi\left(v_{k}, v_{k+d / 2}\right) \\
& =2 \sum_{k=1}^{d / 2}\left|a_{k}\right|^{2} \operatorname{Tr}_{E / \mathbb{Q}}\left(\zeta \phi\left(v_{k}, v_{k+d / 2}\right)\right)=2[E: \mathbb{Q}] \sum_{k=1}^{d / 2}\left|a_{k}\right|^{2}
\end{aligned}
$$

which proves that $x \mapsto \psi(x, i \bar{x})$ is positive definite on the subspace $V_{0}^{1,0}$. The Hodge structure $V_{0} \otimes_{\mathbb{Q}} E$ therefore belongs to $D^{\mathrm{sp}}$ as desired.

Proof of Theorem 81. Let $\theta: A \rightarrow \hat{A}$ be the polarization on $A$. As before, let $\mathcal{M}$ be the moduli space of abelian varieties of dimension $d / 2$, with polarization of the same type as $\theta$, and level 3structure. Then $\mathcal{M}$ is a quasi-projective complex manifold, and the period domain $D$ is its universal covering space (with the Hodge structure $H^{1}(A)$ mapping to the point $A$ ). Let $B \subseteq \mathcal{M}$ be the locus of those abelian varieties whose endomorphism algebra contains $E$. Note that the original abelian variety $A$ is contained in $B$. Since every element $e \in E$ is a Hodge class in $\operatorname{End}(A) \otimes \mathbb{Q}$, it is clear that $B$ is a Hodge locus; in particular, $B$ is a quasi-projective variety by the theorem of Cattani-Deligne-Kaplan. As before, we let $\pi: \mathcal{A} \rightarrow B$ be the restriction of the universal family of abelian varieties to $B$.

Now we claim that the preimage of $B$ in $D$ is precisely the set $D^{\text {sp }}$ of Hodge structures of split Weil type. Indeed, the endomorphism ring of any Hodge structure in the preimage of $B$ contains $E$ by construction; since it is also polarized by the form $\psi$, all the conditions in Definition 76 are satisfied, and so the Hodge structure in question belongs to $D^{\text {sp }}$. Because $D$ is the universal covering space of $\mathcal{M}$, this implies in particular that $B$ is connected and smooth, hence a quasi-projective complex manifold.

The first two assertions are obvious from the construction, whereas the third follows from Lemma 83. This concludes the proof.

To complete the proof of Deligne's theorem, we have to show that every split Weil class is an absolute Hodge class. For this, we argue as follows. Consider the family of abelian varieties $\pi: \mathcal{A} \rightarrow B$ from Theorem 81. By Proposition 77 the space of split Weil classes $\bigwedge_{E}^{d} H^{1}\left(\mathcal{A}_{t}, \mathbb{Q}\right)$ consists of Hodge classes for every $t \in B$. The family also contains an abelian variety of the form $A_{0} \otimes_{\mathbb{Q}} E$, and according to Proposition [80, all split Weil classes on this particular abelian variety are absolute. But now $B$ is irreducible, and so Principle B applies and shows that for every $t \in B$, all split Weil classes on $\mathcal{A}_{t}$ are absolute. This finishes the third step of the proof, and completely establishes Deligne's theorem. 


\section{REFERENCES}

[1] André, Y.: Pour une théorie inconditionnelle des motifs, Pub. Math. IHÉS 83, pp. 5-49 (1996).

[2] André, Y.: Une remarque à propos des cycles de Hodge de type CM, in Séminaire de Théorie des Nombres, Paris, 1989-90. Progress in Mathematics, 102, Birkhäuser Boston, Boston, MA. pp. 1-7 (1992).

[3] André, Y.: Une introduction aux motifs (motifs purs, motifs mixtes, périodes), Panoramas et Synthèses 17, Société Mathématique de France (2004).

[4] Blasius, D.: A p-adic property of Hodge classes on abelian varieties, in Motives (Seattle, WA, 1991), pp. 293-308, Proc. Sympos. Pure Math. 55, Part 2, Amer. Math. Soc., Providence, RI (1994).

[5] Bloch, S.: Semi-regularity and de Rham cohomology, Invent. Math. 17, pp. 51-66 (1972).

[6] Bourbaki, N.: Éléments de mathématique. Première partie: les structures fondamentales de l'analyse. Livre II: Algèbre. Chapitre 9: Formes sesquilinéaires et formes quadratiques, Actualités Sci. Ind. n. 1272 Hermann, Paris 1959.

[7] Brosnan, P., Pearlstein, G.: On the algebraicity of the zero locus of an admissible normal function, preprint, arxiv.org/abs/0910.0628.

[8] Brosnan, P., Pearlstein, G., Schnell, C.: The locus of Hodge classes in an admissible variation of mixed Hodge structure, C. R. Acad. Sci. Paris, Ser. I 348, pp. 657-660 (2010).

[9] Carlson, J.: Extensions of mixed Hodge structures, in Journées de Géometrie Algébrique d'Angers, juillet 1979, pp. 107-127, Sijthoff \& Noordhoff, Alphen aan den Rijn-Germantown, Md., 1980.

[10] Cattani, E., Deligne, P., Kaplan, A.: On the locus of Hodge classes, J. Amer. Math. Soc. 8, n. 2, pp. 483-506 (1995).

[11] Charles, F.: Conjugate varieties with distinct real cohomology algebras, J. Reine Angew. Math. 630, pp. 125-139 (2009).

[12] Charles, F.:On the zero locus of normal functions and the étale Abel-Jacobi map, Int. Math. Res. Not. IMRN 2010 n. 12 , pp. 2283-2304.

[13] Deligne, P.: Théorie de Hodge, II, Inst. Hautes Études Sci. Publ. Math. No. 40 (1971), pp. 5--57.

[14] Deligne, P.: La conjecture de Weil pour les surfaces K3 Invent. Math. 15, pp. 206-226 (1972).

[15] Deligne, P.: Théorie de Hodge, III, Inst. Hautes Études Sci. Publ. Math. No. 44 (1974), pp. 5--77.

[16] Deligne, P.: Hodge cycles on abelian varieties (notes by J. S. Milne), in Lecture Notes in Mathematics 900 (1982), pp. 9-100, Springer-Verlag.

[17] Deligne, P., Milne, J.: Tannakian categories, in Lecture Notes in Mathematics 900 (1982), pp. 101-220, SpringerVerlag.

[18] Fulton, W., Harris, J.: Representation theory. A first course, Graduate Texts in Mathematics 129, Readings in Mathematics. Springer-Verlag, New York, 1991.

[19] van Geemen, B.: Kuga-Satake varieties and the Hodge conjecture, in The arithmetic and geometry of algebraic cycles (Banff, AB, 1998), pp. 51--82, NATO Sci. Ser. C Math. Phys. Sci., 548, Kluwer Acad. Publ., Dordrecht, 2000.

[20] Grothendieck, A.: On the de Rham cohomology of algebraic varieties, Pub. Math. IHÉS 29, pp. 95-103 (1966).

[21] Grothendieck, A.: Standard conjectures on algebraic cycles, Algebraic Geometry (Internat. Colloq., Tata Inst. Fund. Res., Bombay, 1968) pp. 193-199 Oxford Univ. Press, London (1969).

[22] Jannsen, U.: Mixed motives and algebraic K-theory, Lecture Notes in Mathematics, 1400, Springer-Verlag, Berlin, 1990.

[23] Katz, N. M., Oda, T.: On the differentiation of de Rham cohomology classes with respect to parameters, J. Math. Kyoto Univ. 8 (1968), pp. 199--213.

[24] Kuga, M., Satake, I.: Abelian varieties attached to polarized K3 surfaces, Math. Ann. 169, pp. $239-242$ (1967).

[25] Landherr, W.: Äquivalenz Hermitescher Formen über einem beliebigen algebraischen Zahlkörper, Abhandlungen des Mathematischen Seminars der Universität Hamburg 11, pp. 245-248 (1936).

[26] Morrison, D.: The Kuga-Satake variety of an abelian surface, J. Algebra 92 (1985) pp. 454-476.

[27] Paranjape, K.: Abelian varieties associated to certain K3 surfaces, Compositio Math. 68 (1988), n. 1, pp. $11--22$.

[28] Voisin, C.: Théorie de Hodge et géométrie algébrique complexe, Cours Spécialisés, vol. 10, Société Mathématique de France, Paris (2002).

[29] Voisin, C.: A counterexample to the Hodge conjecture extended to Kähler varieties, Int. Math. Res. Not. 2002, n. 20, pp. 1057-1075. 
[30] Voisin, C.: A generalization of the Kuga-Satake construction, Pure Appl. Math. Q. 1 (2005), n. 3, part 2, pp. $415-439$.

[31] Voisin, C.: Hodge loci and absolute Hodge classes, Compos. Math. 143 (2007), n. 4, pp. 945-958.

[32] Voisin, C.: Some aspects of the Hodge conjecture, Jpn. J. Math. 2 (2007), n. 2, pp. 261-296.

[33] Voisin, C.: Hodge loci, preprint 2010.

Département de Mathématiques et Applications, École Normale Supérieure, 45, Rue d’Ulm, 75005 Paris, France

E-mail address: francois.charles@ens.fr

Department of Mathematics, Statistics and Computer Science, University of Illinois at Chicago, 851 S. Morgan Street, Chicago, IL 60607, USA

E-mail address: cschnell@math.uic.edu 